\title{
Connected components of the compactification of representation spaces of surface groups
}

\author{
MAXIME WOLFF
}

\begin{abstract}
The Thurston compactification of Teichmüller spaces has been generalised to many different representation spaces by Morgan, Shalen, Bestvina, Paulin, Parreau and others. In the simplest case of representations of fundamental groups of closed hyperbolic surfaces in $\operatorname{PSL}(2, \mathbb{R})$, we prove that this compactification behaves very badly: the nice behaviour of the Thurston compactification of the Teichmüller space contrasts with wild phenomena happening on the boundary of the other connected components of these representation spaces. We prove that it is more natural to consider a refinement of this compactification, which remembers the orientation of the hyperbolic plane. The ideal points of this compactification are oriented $\mathbb{R}$-trees, ie, $\mathbb{R}$-trees equipped with a planar structure.
\end{abstract}

53C23; 20H10, 32G15

\section{Introduction}

Let $\Gamma$ be a discrete group with a given finite generating set $S$. In all this text, we consider the space $R_{\Gamma}(n)=\operatorname{Hom}\left(\Gamma\right.$, Isom $\left.{ }^{+}\left(\mathbb{H}^{n}\right)\right)$ of actions of $\Gamma$ on the real hyperbolic space of dimension $n$ by isometries preserving the orientation. The set $R_{\Gamma}(n)$ naturally embeds in (Isom $\left.{ }^{+}\left(\mathbb{H}^{n}\right)\right)^{S}$, giving it a Hausdorff, locally compact topology. The Lie groups Isom ${ }^{+}\left(\mathbb{H}^{n}\right)$ and $\operatorname{Isom}\left(\mathbb{H}^{n}\right)$ act on $R_{\Gamma}(n)$ by conjugation, and we will consider the quotients $R_{\Gamma}(n) / \operatorname{Isom}\left(\mathbb{H}^{n}\right)$ and $R_{\Gamma}(n) / \operatorname{Isom}^{+}\left(\mathbb{H}^{n}\right)$, equipped with their quotient topologies.

We will mainly focus on the case when $\Gamma=\pi_{1} \Sigma_{g}$ is the fundamental group of a closed, oriented, connected surface of genus $g \geq 2$, with a given standard presentation (ie, a marking); we then denote $R_{g}(n)=R_{\pi_{1} \Sigma_{g}}(n)$. Also, we are mainly interested in the case $n=2$ (thus $\operatorname{Isom}^{+}\left(\mathbb{H}^{2}\right)=\operatorname{PSL}(2, \mathbb{R})$ ), and we denote $R_{g}=R_{g}(2)$.

The space $R_{g}$ is a real algebraic variety (see Culler and Shalen [9]), and is smooth, of dimension $6 g-3$ outside the set of abelian representations (see Weil [42] and Goldman [19]). Outside the set of (classes of) elementary representations (ie, having a global fixed point in $\left.\mathbb{H}^{2} \cup \partial \mathbb{H}^{2}\right)$, the quotient space $R_{g} / \operatorname{PSL}(2, \mathbb{R})$, equipped with 
the quotient topology, is again smooth of dimension $6 g-6$; one of its connected components is naturally identified with the Teichmüller space of the surface (see Goldman $[18 ; 20])$.

A function $e: R_{g} \rightarrow \mathbb{Z}$, called the Euler class, plays a key role in understanding the spaces $R_{g}$ and $R_{g} / \operatorname{PSL}(2, \mathbb{R})$. There are several ways to define it; for instance, a representation $\rho \in R_{g}$ defines a circle bundle on $\Sigma_{g}$, which has a $\mathbb{Z}$-valued characteristic class, the Euler class. We will review this Euler class and its properties in detail in Section 2.3, and also refer to Milnor [30], Wood [44], Ghys [14], Matsumoto [28], Goldman [20] and Calegari [6] for a deep understanding of this class.

In 1988, W Goldman proved [20] that $R_{g}$ has $4 g-3$ connected components, which are the preimages $e^{-1}(k)$ for $2-2 g \leq k \leq 2 g-2$ (the fact that the absolute value of the Euler class is bounded by $2 g-2$ was previously known as the Milnor-Wood inequality [30; 44]). He also proved that the space $R_{g}(3)=\operatorname{Hom}\left(\pi_{1} \Sigma_{g}, \operatorname{PSL}(2, \mathbb{C})\right)$ has two connected components. The elements of $R_{g}$ of even Euler class, on one hand, and the ones of odd Euler class on the other hand, fall into these two different components of $R_{g}(3)$. The Euler class is still well-defined in the quotient space $R_{g} / \operatorname{PSL}(2, \mathbb{R})$ (we denote e: $R_{g} / \operatorname{PSL}(2, \mathbb{R}) \rightarrow \mathbb{Z}$ ), whence the space $R_{g} / \operatorname{PSL}(2, \mathbb{R})$ also admits $4 g-3$ connected components, similarly. However, only the absolute value of the Euler class is defined on $R_{g} / \operatorname{Isom}\left(\mathbb{H}^{2}\right)$, which has $2 g-1$ connected components. The main theorem of Goldman's thesis [18] states that a representation $\rho \in R_{g}$ has extremal Euler class $(|e(\rho)|=2 g-2)$ if and only if $\rho$ is discrete and faithful. It follows that, in $R_{g} / \operatorname{PSL}(2, \mathbb{R})$, the connected component $\mathbf{e}^{-1}(2-2 g)$ is naturally identified with the Teichmüller space of $\Sigma_{g}$.

In 1976, W Thurston [41] introduced a natural compactification of Teichmüller spaces; his construction was intensively studied and detailed by Fathi, Laudenbach and Poénaru [11]. The data of the lengths of all geodesic closed curves suffices to determine a hyperbolic structure, and, essentially, W Thurston's compactification consists in considering points in the Teichmüller space as sets of lengths of curves, and then embedding them in a projective space (thus, considering lengths only up to a scalar), in which the Teichmüller space has a relatively compact image. A very important feature of this compactification is that the boundary added to the Teichmüller space is homeomorphic to a sphere of dimension $6 g-7$, in such a way that the Thurston compactification of the Teichmüller space is homeomorphic to a closed ball of dimension $6 g-6$.

Thurston's compactification has been extended to other connected components of $R_{g} / \operatorname{PSL}(2, \mathbb{R})$, and to other representation spaces in successive works. In 1984, J Morgan and P Shalen [32], using techniques of algebraic geometry, defined a compactification of the real algebraic variety $X_{\Gamma, \mathrm{SL}(2, \mathbb{R})}$ of characters of representations 
of $\Gamma$ in $\operatorname{SL}(2, \mathbb{R})$. We will denote this compactification by ${\overline{X_{\Gamma, \mathrm{SL}(2, \mathbb{R})}}}^{\mathrm{MS}}$, and by $\bar{X}_{g, \mathrm{SL}(2, \mathbb{R})} \mathrm{MS}$ in the case $\Gamma=\pi_{1} \Sigma_{g}$. Since all the representations of even Euler class in $\operatorname{PSL}(2, \mathbb{R})$ (and, in particular, elements of the Teichmüller space) lift to $\operatorname{SL}(2, \mathbb{R})$ (see eg [20]), this defines a compactification of Teichmüller spaces, and it coincides with Thurston's. In [31], J Morgan generalized this construction to the group $\operatorname{SO}(n, 1)$ for $n \geq 2$. In 1988, M Bestvina [4] and F Paulin [35], independently, gave a much more geometric viewpoint of this compactification, for representations in $\operatorname{Isom}^{+}\left(\mathbb{H}^{n}\right), n \geq 2$. In $[36 ; 35]$, F Paulin defined and studied a very natural topology, called the equivariant Gromov topology, for spaces of actions of a given discrete group on metric spaces. He proved that the quotient topology, on the space $R_{\Gamma}(n) / \operatorname{Isom}\left(\mathbb{H}^{n}\right)$, coincides with the equivariant Gromov topology (this will be reviewed in Section 2.2). Equipped with this topology, the space $m_{\Gamma}^{\mathrm{fd}}(n)$ of (conjugacy classes of) faithful and discrete representations has a natural compactification, which recovers the compactification of [32; 31]. The ideal points of this compactification are actions of $\Gamma$ on $\mathbb{R}$-trees. Note, finally, that A Parreau extended [33] this compactification to representation spaces in higher rank groups.

This compactification being well-defined, it is a natural question to ask if it respects the topology and geometry of $R_{g} / \operatorname{PSL}(2, \mathbb{R})$, as does the Thurston compactification, when restricted to the Teichmüller space alone. We shall prove this is not the case, and that this compactification, as defined in all the works mentioned above, leads to a very wild space.

The works of J Morgan and P Shalen, of M Bestvina and of F Paulin all yield the same compactification, and we shall follow the approach of F Paulin, in which it is easier to add a notion of orientation. In order to use F Paulin's construction, we first define explicitly (see Section 2.1) the biggest Hausdorff quotients of $R_{\Gamma}(n) / \operatorname{Isom}\left(\mathbb{H}^{n}\right)$ and $R_{\Gamma}(n) /$ Isom $^{+}\left(\mathbb{H}^{n}\right)$, that we denote $m_{\Gamma}^{u}(n)$ and $m_{\Gamma}^{o}(n)$, respectively (indeed, these spaces are not Hausdorff in general, so that M Bestvina and F Paulin's constructions cannot be extended literally to these spaces). Again, we write $m_{\Gamma}^{u}=m_{\Gamma}^{u}(2), m_{g}^{u}(n)=$ $m_{\pi_{1} \Sigma_{g}}(n)$ and so on. Note that, in the space $m_{g}^{u}$, only the absolute value of the Euler class is still defined, and $m_{g}^{u}$ has $2 g-1$ connected components. We denote by $\overline{m_{g}^{u}(n)}$ the compactification of $m_{g}^{u}(n)$ as it is constructed by F Paulin.

Also, in all this text, for all $k \in\{2-2 g, \ldots, 2 g-2\}$, we will denote by $m_{g, k}^{o}$ the subset of $m_{g}^{o}$ consisting of classes of representations of Euler class $k$, and for $k \in$ $\{0, \ldots, 2 g-2\}, m_{g, k}^{u}$ will denote the connected component of $m_{g}^{u}$ consisting of classes of representations whose Euler class, in absolute value, equals $k$.

Theorem 1.1 Let $g \geq 4$ and $k \in\{0, \ldots, 2 g-3\}$. Then, in $\overline{m_{g}^{u}}$, the boundary of the Teichmüller space, $\partial m_{g, 2 g-2}^{u}$, is contained in $\partial m_{g, k}^{u}$ as a closed, nowhere dense subset. 
In particular:

Corollary 1.2 For all $g \geq 4, \overline{m_{g}^{u}}$ is connected.

We also prove that for $g=2$ and $g=3$, the space $\overline{m_{g}^{u}}$ possesses at most two connected components. Actually, Theorem 1.1 should hold for all $g \geq 2$, but the proof presented here requires that $g \geq 4$.

In particular, the two connected components of $\operatorname{Hom}\left(\pi_{1} \Sigma_{g}, \operatorname{PSL}(2, \mathbb{C})\right) / \operatorname{PSL}(2, \mathbb{C})$ meet at their boundary in this compactification, as soon as $g \geq 3$ :

Corollary 1.3 For all $g \geq 3$, the space $\overline{m_{g}^{u}(3)}$ is connected.

If we consider only representations of even Euler class (ie, that lift to $\operatorname{SL}(2, \mathbb{R})$ ) then the result holds for all $g \geq 2$ :

Corollary 1.4 For all $g \geq 2$, the space ${\overline{X_{g, S L}(2, \mathbb{R})}}^{\mathrm{MS}}$ is connected.

Theorem 1.1 not only implies that the space $\overline{m_{g}^{u}}$ is connected, but it also implies that this space is extremely wild. Since the connected components $m_{g, k}^{u}$ are of dimension $6 g-6$ (see Weil [42] or Goldman [19, Section 1]), one should expect that the boundary $\partial m_{g, k}^{u}$ has dimension at most $6 g-7$. However, the boundary of the Teichmüller space itself has dimension $6 g-7$ (see eg [11, Exposé 1, Théorème 1]). Therefore, it follows from Theorem 1.1 that the compactifications $\overline{m_{g, k}^{u}}$ of the "exotic" connected components (ie, the connected components $m_{g, k}^{u}$ such that $|k| \leq 2 g-3$ ) have no PL structures, or cell complex structures or so on, compatible with the compactification. This contrasts very strongly with the behaviour of the Thurston compactification of the Teichmüller spaces.

The proof of Theorem 1.1 uses the following fact, interesting for itself:

Proposition 1.5 The connected components of $m_{g}^{o}$ and $m_{g}^{u}$ are one-ended.

Actually, for all $k \neq 0$, this follows from a theorem of $\mathrm{N}$ Hitchin (see [23, Proposition 10.2]), which says that the connected component $m_{g, k}^{u}$ is homeomorphic to a complex vector bundle over the $(2 g-2-|k|)$-th symmetric product of the surface $\Sigma_{g}$. However, the proof of Proposition 1.5 is far more simple and extends to the case $k=0$.

The wild behaviour of the compactification of $m_{g}^{u}$ is due (at least) to the fact that the equivariant Gromov topology forgets the orientation of the space $\mathbb{H}^{n}$. This information, in the case $n=2$, is the information carrying the Euler class, and which separates the 
space $m_{g}^{u}$ into its connected components. By restoring this orientation, we define a new compactification of these representation spaces, cancelling (partly, at least) this wildness.

We define a notion of convergence in the sense of Gromov for oriented spaces, which preserves the orientation. This enables us to define a new compactification of $m_{\Gamma}^{o}$, in which the ideal points, added at the boundary, are oriented $\mathbb{R}$-trees, which form a set $\mathcal{T}^{o}(\Gamma)$ :

Theorem 1.6 The map $m_{\Gamma}^{o} \rightarrow m_{\Gamma}^{o} \cup \mathcal{T}^{o}(\Gamma)$ induces a natural compactification of $m_{\Gamma}^{o}$. Moreover, the natural map $\pi: \overline{m_{\Gamma}^{o}} \rightarrow \overline{m_{\Gamma}^{u}}$, which consists in forgetting the orientation, is onto, and some of its fibres have the same cardinality as $\mathbb{R}$.

By natural, we mean, following F Paulin [37], that the action of the group Out( $\Gamma$ ) (or the mapping class group, if $\Gamma$ is a surface group) on $m_{\Gamma}^{o}$ extends continuously to the compact space $\overline{m_{\Gamma}^{o}}$.

One can define an Euler class in a quite general context, as we will see in Section 2.3. In particular, the actions of $\pi_{1} \Sigma_{g}$ on oriented $\mathbb{R}$-trees admit an Euler class, and we shall prove the following:

Theorem 1.7 The Euler class e: $\overline{m_{g}^{o}} \rightarrow \mathbb{Z}$ is a continuous function. In particular, the compactification $\overline{m_{g}^{o}}$ possesses as many connected components as the space $m_{g}^{o}$.

By Theorem 1.6, it is a necessary condition, for an action of $\pi_{1} \Sigma_{g}$ on an $\mathbb{R}$-tree to be in $\overline{m_{g}^{u}}$, to preserve some orientation. In particular, we can construct explicit actions on $\mathbb{R}$-trees which are not the limit of actions of surface groups on $\mathbb{H}^{2}$. These explicit actions can even be obtained as limits of actions of surface groups on $\mathbb{H}^{3}$, so that we get the following proposition:

Proposition 1.8 Let $g \geq 3$. There exist minimal actions of $\pi_{1} \Sigma_{g}$ on $\mathbb{R}$-trees by isometries, which are in $\partial \overline{m_{g}^{u}(3)}$ but not in $\partial \overline{m_{g}^{u}(2)}$.

This contrasts again with the case of discrete and faithful representations. Indeed, R Skora [40] proved that a minimal action of $\pi_{1} \Sigma_{g}$ on an $\mathbb{R}$-tree has small arc stabilisers (ie, the stabiliser of any pair of distinct points of the tree is virtually abelian) if and only if it is the limit of discrete and faithful representations of $\pi_{1} \Sigma_{g}$ in $\operatorname{PSL}(2, \mathbb{R})$ (or equivalently, it is a point in the boundary of the Teichmüller space), and it is well-known (see eg $[32 ; 35 ; 4]$ ) that limits of discrete and faithful representations in $\operatorname{Isom}^{+}\left(\mathbb{H}^{n}\right)$ enjoy that property. As a corollary of R Skora's result, we thus have 
$\partial \overline{m_{g}^{\mathrm{fd}}(n)}=\partial \overline{m_{g}^{\mathrm{fd}}(2)}$. When we consider representations which may not be faithful and discrete, Proposition 1.8 states that this equality does not hold any more.

Note that C McMullen [29] has developed, independently, a theory of oriented $\mathbb{R}$-trees, under the name of ribbon $\mathbb{R}$-trees, in order to compactify the set of proper holomorphic maps from the unit disk $\Delta \subset \mathbb{C}$ into itself.

This text is organised as follows. Section 2 gathers every background material concerning the spaces that we wish to compactify. Section 2.1 is devoted to the explicit construction of $m_{\Gamma}^{u}(n)$ and $m_{\Gamma}^{o}(n)$, the biggest Hausdorff quotients of $R_{\Gamma}(n) / \operatorname{Isom}\left(\mathbb{H}^{n}\right)$ and $R_{\Gamma}(n) /$ Isom $^{+}\left(\mathbb{H}^{n}\right)$. In Section 2.2, we review F Paulin's point of view on the compactification of $m_{\Gamma}^{u}(n)$, while adapting it slightly so that it indeed defines a compactification of the whole space $m_{\Gamma}^{u}(n)$. In Section 2.3, we recall the construction of the Euler class, and establish a technical lemma which will enable us to prove that the Euler class extends continuously to the boundary (in the oriented compactification) of $m_{g}^{o}$ (Theorem 1.7). Section 2.4 recalls an argument of Z Sela in the context of limit groups, which plays a key role in the proof of Theorem 1.1. Section 3 contains the core of our results on the compactification. It begins with the proof of Proposition 1.5, then turns to the degenerations of $\overline{m_{g}^{u}}$, and finally we construct the oriented compactification.

Acknowledgements This article, essentially, gathers the main results of my $\mathrm{PhD}$ thesis, directed by Louis Funar; he inspired this work from the beginning and suggested several ideas developed here. I am very grateful to Vincent Guirardel, Gilbert Levitt, Frédéric Paulin and the anonymous referee, who corrected some mistakes in earlier versions of this text, and whose numerous remarks have deeply improved the presentation of this paper. I am grateful to the editor Walter Neumann and the anonymous referee for their kindness and their patience. I would also like to thank Thierry Barbot, Gérard Besson, Léa Blanc-Centi, Simone Diverio, Elisha Falbel, Damien Gaboriau, Sylvain Gallot, Anne Parreau and Vlad Sergiescu for inspiring discussions and encouragements. I also wish to thank Bill Goldman for sending me a copy of his thesis [18], and the members of the LATP in Marseille and the IMJ in Paris, where I finished the redaction of this paper.

The author was partially supported by the ANR RepSurf grant ANR-06-BLAN-0311.

\section{Preliminaries}

\subsection{The representation spaces}

First note that the quotient topological spaces $m_{\Gamma}^{\prime}(n)=R_{\Gamma}(n) / \operatorname{Isom}^{+}\left(\mathbb{H}^{n}\right)$ and $R_{\Gamma}(n) / \operatorname{Isom}\left(\mathbb{H}^{n}\right)$ are not Hausdorff in general. Indeed, in $\operatorname{Isom}^{+}\left(\mathbb{H}^{2}\right)=\operatorname{PSL}(2, \mathbb{R})$, 
the element

$$
\pm\left(\begin{array}{ll}
1 & 1 \\
0 & 1
\end{array}\right)
$$

is conjugate to

$$
\pm\left(\begin{array}{cc}
1 & 1 / t^{2} \\
0 & 1
\end{array}\right)
$$

for all $t \in \mathbb{R}^{*}$, hence its conjugacy class cannot be separated from the one of the identity. As soon as there exists a morphism of $\Gamma$ onto $\mathbb{Z}$, we can therefore construct abelian representations of $\Gamma$ in $\operatorname{PSL}(2, \mathbb{R})$, and more generally in $\operatorname{Isom}^{+}\left(\mathbb{H}^{n}\right)$, which are not separated from the trivial representation in these quotient spaces. However, in order to use F Paulin's construction of the compactification of these representation spaces, we need to work with Hausdorff spaces.

We are thus going to define explicitly the biggest Hausdorff quotients of $m_{\Gamma}^{\prime}(n)$ and of $R_{\Gamma}(n) / \operatorname{Isom}\left(\mathbb{H}^{n}\right)$. The construction we propose here uses only elementary hyperbolic geometry. It can be generalized by replacing $\mathbb{H}^{n}$ by nonpositively curved symmetric spaces; see Parreau [34; 33].

First we need to fix some notation for the real hyperbolic space $\mathbb{H}^{n}$ and its group of orientation-preserving isometries. We will be using the upper hyperboloid model, and we refer to Benedetti and Petronio [3, Chapter A] for a complete overview.

Equip the space $\mathbb{R}^{n+1}$ with the quadratic form

$$
q\left(x_{0}, x_{1}, \ldots, x_{n}\right)=2 x_{0} x_{1}+x_{2}^{2}+\cdots+x_{n}^{2} .
$$

The subspace $\left\{\underline{x} \in \mathbb{R}^{n+1} \mid q(\underline{x})=-1\right\}$ of $\mathbb{R}^{n+1}$ has two connected components, and we define

$$
\mathbb{H}^{n}=\left\{\underline{x} \in \mathbb{R}^{n+1} \mid q(\underline{x})=-1, x_{1}-x_{0}>0\right\} .
$$

The form $\langle\underline{x}, \underline{y}\rangle=x_{0} y_{1}+x_{1} y_{0}+x_{2} y_{2}+\cdots+x_{n} y_{n}$ defines a scalar product on the tangent space at each point of $\mathbb{H}^{n}$, hence a Riemannian metric on $\mathbb{H}^{n}$. The images of the geodesics of this space are its intersections with the (linear) planes of $\mathbb{R}^{n+1}$.

Denote by $\mathrm{SO}_{\mathbb{R}}(n, 1)$ the subgroup of $\operatorname{SL}(n+1, \mathbb{R})$ consisting of elements preserving $q$. Denote by $\mathrm{SO}_{\mathbb{R}}^{+}(n, 1)$ the index 2 subgroup of $\operatorname{SO}_{\mathbb{R}}(n, 1)$ formed by elements preserving $\mathbb{H}^{n}$ (the other elements exchange the two connected components of $q^{-1}(-1)$ ). Then $\mathrm{SO}_{\mathbb{R}}^{+}(n, 1)$ is the group of orientation-preserving isometries of $\mathbb{H}^{n}$.

The image of $\mathbb{H}^{n}$ on the hyperplane $\left\{x_{0}-x_{1}=0\right\}$, under the stereographic projection of centre $(1 / \sqrt{2},-1 / \sqrt{2}, 0, \ldots, 0)$ is an open disk of center 0 and radius 1 ; we denote it by $\mathbb{D}^{n}$. This yields the usual compactification of $\mathbb{H}^{n}$, and the projection of any geodesic of $\mathbb{H}^{n}$ on $\mathbb{D}^{n}$ is a geodesic in $\mathbb{D}^{n}$ for the Poincaré metric. 
In $\mathrm{SO}_{\mathbb{R}}^{+}(n, 1)$, the stabilizer of the point $(1 / \sqrt{2}, 1 / \sqrt{2}, 0, \ldots, 0) \in \partial \mathbb{D}^{n}$ is the subgroup formed by matrices of the form

$$
\left(\begin{array}{ccc}
\lambda & 0 & (0) \\
r & 1 / \lambda & Y \\
Z & (0) & A
\end{array}\right),
$$

with $\lambda>0, A \in \mathrm{SO}_{\mathbb{R}}(n-1),\|Y\|^{2}=-2 r / \lambda$ and $A^{t} Y=-(1 / \lambda) Z$. The subgroup of elements which also fix the point $(-1 / \sqrt{2},-1 / \sqrt{2}, 0, \ldots, 0) \in \partial \mathbb{D}^{n}$ (and in particular, which preserve globally the geodesic $\mathbb{H}^{n} \cap\left\{x_{2}=\cdots=x_{n}=0\right\}$ ) is formed by matrices of the form

$$
\left(\begin{array}{ccc}
\lambda & 0 & (0) \\
0 & 1 / \lambda & (0) \\
(0) & (0) & A
\end{array}\right),
$$

with $\lambda>0$ and $A \in \mathrm{SO}_{\mathbb{R}}(n-1)$. By analogy with the classical case $n=2$, and with the model of the upper half plane, we denote by 0 the point $(1 / \sqrt{2}, 1 / \sqrt{2}, 0, \ldots, 0) \in \partial \mathbb{D}^{n}$, and the point $(-1 / \sqrt{2},-1 / \sqrt{2}, 0, \ldots, 0) \in \partial \mathbb{D}^{n}$ is denoted by $\infty$.

Elements of $\mathrm{SO}_{\mathbb{R}}^{+}(n, 1)$ having a fixed point in $\mathbb{H}^{n}$ are called elliptic (note in particular that the identity is elliptic: this convention differs from some textbooks such as Katok [25] as well as our thesis [43]), elements with a unique fixed point in $\partial \mathbb{H}^{n}$ are called parabolic, and elements $\varphi \in \operatorname{SO}_{\mathbb{R}}^{+}(n, 1)$ such that $\inf _{x \in \mathbb{H}^{n}} d(x, \varphi(x))>0$ (this lower bound is then achieved) are called loxodromic. Loxodromic isometries fix two points in $\partial \mathbb{H}^{n}$, and are conjugate to a matrix of the form

$$
\left(\begin{array}{ccc}
\lambda & 0 & (0) \\
0 & 1 / \lambda & (0) \\
(0) & (0) & A
\end{array}\right) .
$$

When $A=\mathrm{Id}$, we also say that $\varphi$ is hyperbolic.

The lower bound

$$
d(u)=\inf _{x \in \mathbb{H}^{n}} d(x, u \cdot x)
$$

is achieved if and only if $u$ is nonparabolic. In that case we denote by $\min (u)$ the set $\left\{x \in \mathbb{H}^{n} \mid d(x, u \cdot x)=d(u)\right\}$, and if $r>0$, the set $\left\{x \in \mathbb{H}^{n} \mid d(x, u \cdot x)<r+d(u)\right\}$ is denoted by $\min _{r}(u)$.

In order to prove the local compactness of the quotients $m_{\Gamma}^{o}(n)$ and $m_{\Gamma}^{u}(n)$, we will use the following well-known fact, which says that $\min _{r}(u)$ is at bounded distance of $\min (u)$ whenever $u$ is nonparabolic.

Fact 2.1 Let $u \in \operatorname{Isom}^{+}\left(\mathbb{H}^{n}\right)$ be a nonparabolic element. Then for all $r>0$, there exists $k>0$ such that for all $x \in \min _{r}(u)$ we have $d(x, \min (u))<k$. 
Also, we shall use the following classical property:

Fact 2.2 For every $x \in \mathbb{H}^{n}$, and every $d \in \mathbb{R}$, the set

$$
\left\{\gamma \in \operatorname{Isom}\left(\mathbb{H}^{n}\right) \mid d(x, \gamma x) \leq d\right\}
$$

is compact.

Now we can define the space $m_{\Gamma}^{o}(n)$.

Definition 2.3 We denote by $m_{\Gamma}^{o}(n)$ the subspace of $m_{\Gamma}^{\prime}(n)$ formed by classes of representations which have either 0 , or at least 2 global fixed points in $\partial \mathbb{H}^{n}$.

We have an inclusion $i: m_{\Gamma}^{o}(n) \hookrightarrow m_{\Gamma}^{\prime}(n)$. We can also define a map $\pi: m_{\Gamma}^{\prime}(n) \rightarrow$ $m_{\Gamma}^{o}(n)$ as follows. If $c \in m_{\Gamma}^{o}(n)$, put $\pi(c)=c$ (in particular, $\pi$ is onto). If $c=[\rho] \in$ $m_{\Gamma}^{\prime}(n) \backslash m_{\Gamma}^{o}(n)$, then $\rho$ has a unique fixed point $r_{1} \in \partial \mathbb{H}^{n}$. Choose another point $r_{2} \in \partial \mathbb{H}^{n} \backslash\left\{r_{1}\right\}$ arbitrarily, and denote by $g_{k} \in \mathrm{SO}_{\mathbb{R}}^{+}(n, 1)$ the hyperbolic isometry of axis $\left(r_{1}, r_{2}\right)$, and attractive point $r_{1}$, with translation distance $k$.

Lemma 2.4 The sequence $\left(g_{k}^{-1} \rho g_{k}\right)_{k \in \mathbb{N}}$ converges to a representation $\rho_{\infty} \in R_{\Gamma}(n)$ such that $\left[\rho_{\infty}\right] \in m_{\Gamma}^{o}(n)$, and such that $\left[\rho_{\infty}\right]$ depends neither on the choice of $\rho$ in the conjugacy class $c$ nor of the choice of $r_{2}$.

We can therefore set $\pi(c)=\left[\rho_{\infty}\right]$.

Proof Choose a representant $\rho$ of the conjugacy class $c$ so that $\rho$ fixes $0 \in \partial \mathbb{H}^{n}$ (in other words, conjugate $\rho$ by an isometry sending $r_{1}$ to 0 ). Take $r_{2}=\infty$. Then for all $\gamma \in \Gamma, \rho(\gamma)$ is of the form

$$
\rho(\gamma)=\left(\begin{array}{ccc}
\lambda(\gamma) & 0 & (0) \\
r(\gamma) & 1 / \lambda(\gamma) & Y(\gamma) \\
Z(\gamma) & (0) & A(\gamma)
\end{array}\right) .
$$

In this basis, $g_{k}$ has the form

$$
g_{k}=\left(\begin{array}{lll}
t_{k} & & (0) \\
& 1 / t_{k} & \\
(0) & & \mathrm{I}_{n-1}
\end{array}\right),
$$

where $t_{k} \rightarrow 0$ as $k \rightarrow+\infty$. Then $g_{k}^{-1} \rho g_{k}$ converges to the representation $\rho_{\infty}$ such that for all $\gamma \in \Gamma$,

$$
\rho_{\infty}(\gamma)=\left(\begin{array}{lll}
\lambda(\gamma) & & (0) \\
& 1 / \lambda(\gamma) & \\
(0) & & A(\gamma)
\end{array}\right),
$$

which indeed fixes the points 0 and $\infty$ at the boundary. 
Choosing another representant $\rho^{\prime}$ of $c$ fixing 0 would simply be the same as considering a conjugate $\rho^{\prime}=h^{-1} \rho h$, where $h \in \mathrm{SO}_{\mathbb{R}}^{+}(n, 1)$ has the form

$$
h=\left(\begin{array}{ccc}
\lambda & 0 & (0) \\
r & 1 / \lambda & Y \\
Z & (0) & A
\end{array}\right),
$$

and conjugation by $h$ does not touch the elements $\lambda(\gamma)$ and $A(\gamma)$, which determine the representation $\rho_{\infty}$. Finally, the choice of another $r_{2}$ amounts to conjugate $\rho$ by an orientation-preserving isometry of $\mathbb{H}^{n}$ fixing 0 ; we have just dealt with this case.

It follows that we can equip the set $m_{\Gamma}^{o}(n)$ both with the induced topology, and with the final topology determined by the map $\pi: m_{\Gamma}^{\prime}(n) \rightarrow m_{\Gamma}^{o}(n)$. The object of this section is to prove the following.

Theorem 2.5 The induced topology and the quotient topology coincide on $m_{\Gamma}^{o}(n)$ (in particular, $\pi$ is continuous). Moreover, the space $m_{\Gamma}^{o}(n)$ is Hausdorff, and locally compact.

In particular, this topology is also the final topology defined by the map $R_{\Gamma}(n) \rightarrow m_{\Gamma}^{o}(n)$. It follows that $m_{\Gamma}^{o}(n)$ is the biggest Hausdorff quotient of $m_{\Gamma}^{\prime}(n)$, in the following sense:

Definition 2.6 Let $X$ be a topological space. A quotient space $\pi: X \rightarrow X_{S}$ is called the biggest Hausdorff quotient of $X$ if $X_{S}$ is Hausdorff and if for every continuous mapping $f: X \rightarrow Y$ to a Hausdorff space $Y$, there exists a unique function $\bar{f}: X_{S} \rightarrow Y$ such that $f=\pi \circ \bar{f}$.

Note that every topological space $X$ has a biggest Hausdorff quotient, unique up to a canonical homeomorphism. If $x, y \in X$, put $x \sim y$ if $x$ and $y$ have the same image in every Hausdorff quotient of $X$. This defines an equivalence relation, and $X / \sim$ is easily seen to be the biggest Hausdorff quotient of $X$.

The representations which have no fixed points in $\partial \mathbb{H}^{n}$ are called nonparabolic.

Lemma 2.7 (Compare with [33, Proposition 2.6].) The subset of conjugacy classes of nonparabolic representations is open in $m_{\Gamma}^{\prime}(n)$.

Proof Let $\rho \in R_{\Gamma}(n)$ be nonparabolic. Then we must prove that $[\rho]$ possesses, in $m_{\Gamma}^{\prime}(n)$, a neighbourhood consisting of nonparabolic representations. The space $R_{\Gamma}(n) \subset\left(M_{n+1}(\mathbb{R})\right)^{S}$ is a metric space, and the map $R_{\Gamma}(n) \rightarrow m_{\Gamma}^{\prime}(n)$ is open, 
hence every point of $m_{\Gamma}^{\prime}(n)$ has a countable fundamental system of neighbourhoods; it follows that we can use sequential criteria in this space.

Consider $\left(\left[\rho_{k}\right]\right)_{k} \in m_{\Gamma}^{\prime}(n)^{\mathbb{N}}$ such that for all $k, \rho_{k}$ has a fixed point $r_{k} \in \partial \mathbb{H}^{n}$; and suppose (up to conjugating these representations) that $\rho_{k} \rightarrow \rho$ : let us prove that $\rho$ has a fixed point in $\partial \mathbb{H}^{n}$. Up to extracting a subsequence, $r_{k}$ converges to a point $r \in \partial \mathbb{H}^{n}$. Then there exists $h_{k} \in \mathrm{SO}_{\mathbb{R}}^{+}(n, 1)$ such that $h_{k}\left(r_{k}\right)=r$ and such that $h_{k} \rightarrow \mathrm{Id}$. Then $h_{k} \rho_{k} h_{k}^{-1}$ fixes $r$ globally, and converges to $\rho$, hence $\rho$ fixes $r$ globally. It follows that the set of representations which have at least one fixed point in $\partial \mathbb{H}^{n}$ is a closed subset of $m_{\Gamma}^{\prime}(n)$.

The first step towards the continuity of $\pi$ is the following.

Lemma 2.8 Let $\left(\rho_{k}\right)_{k}$ be a sequence of representations, each having a unique fixed point in $\partial \mathbb{H}^{n}$, converging in $R_{\Gamma}(n)$ to a representation $\rho$ which has at least two fixed points in $\partial \mathbb{H}^{n}$. Then $\pi\left(\left[\rho_{k}\right]\right)$ converges to $[\rho]$, in the space $m_{\Gamma}^{\prime}(n)$.

Proof Let us first prove that up to considering a subsequence, the sequence $\pi\left(\left[\rho_{k}\right]\right)$ converges to $[\rho]$. The fixed point $r_{k}$ of $\rho_{k}$ stays in the compact space $\partial \mathbb{H}^{n}$, hence there is a subsequence $r_{\varphi(k)}$ of fixed points of $\rho_{\varphi(k)}$ which converges to a point $r \in \partial \mathbb{H}^{n}$, which is therefore a fixed point of $\rho$. For all $k$, there exists an orientationpreserving isometry $h_{\varphi(k)}$ of $\mathbb{H}^{n}$ such that $h_{\varphi(k)}\left(r_{\varphi(k)}\right)=r$, satisfying $h_{\varphi(k)} \rightarrow$ Id. Then $h_{\varphi(k)}^{-1} \rho_{\varphi(k)} h_{\varphi(k)}$ converges to $\rho$, and fixes $r$. In other words, we may suppose that $\rho_{\varphi(k)}$ fixes $r$, and up to conjugation we can further suppose that $r=0$. Now for all $\gamma \in \Gamma, \rho_{\varphi(k)}(\gamma)$ and $\rho(\gamma)$ are of the form

$$
\rho_{\varphi(k)}(\gamma)=\left(\begin{array}{ccc}
\lambda_{\varphi(k)}(\gamma) & 0 & (0) \\
r_{\varphi(k)}(\gamma) & 1 / \lambda_{\varphi(k)}(\gamma) & Y_{\varphi(k)}(\gamma) \\
Z_{\varphi(k)}(\gamma) & (0) & A_{\varphi(k)}(\gamma)
\end{array}\right), \quad \rho(\gamma)=\left(\begin{array}{ccc}
\lambda(\gamma) & 0 & (0) \\
0 & 1 / \lambda(\gamma) & (0) \\
(0) & (0) & A(\gamma)
\end{array}\right) \text {, }
$$

and by construction, a representant (denote it $\left.\pi \rho_{\varphi(k)}\right)$ of the conjugacy class $\pi\left(\left[\rho_{\varphi(k)}\right]\right)$ has the form

$$
\pi \rho_{\varphi(k)}(\gamma)=\left(\begin{array}{ccc}
\lambda_{\varphi(k)}(\gamma) & 0 & (0) \\
0 & 1 / \lambda_{\varphi(k)}(\gamma) & (0) \\
(0) & (0) & A_{\varphi(k)}(\gamma)
\end{array}\right)
$$

Moreover, $\rho_{\varphi(k)}(\gamma) \rightarrow \rho(\gamma)$ for all $\gamma \in \Gamma$, hence $\lambda_{\varphi(k)}(\gamma) \rightarrow \lambda(\gamma)$ and $A_{\varphi(k)}(\gamma) \rightarrow$ $A(\gamma)$, so that $\pi \rho_{\varphi(k)} \rightarrow \rho$.

The proof we have just given works for every subsequence of the sequence $\left(\rho_{k}\right)_{k}$. In particular, every subsequence of $\left(\pi\left(\left[\rho_{k}\right]\right)\right)_{k}$ possesses a subsequence converging to $[\rho]$. This implies that $\pi\left(\left[\rho_{k}\right]\right)$ converges to $[\rho]$. 
We now define a natural function on $R_{\Gamma}(n)$ which will be very useful: for every $\rho \in R_{\Gamma}(n)$, let

$$
\mathrm{d}(\rho)=\inf _{x \in \mathbb{H}^{n}} \max _{s \in S} d(x, \rho(s) \cdot x) .
$$

Also, for every $\rho \in R_{\Gamma}(n)$, we put

and

$$
\begin{aligned}
\min (\rho) & =\left\{x \in \mathbb{H}^{n} \mid \max _{s \in S} d(x, \rho(s) \cdot x)=\mathrm{d}(\rho)\right\} \\
\min _{\varepsilon}(\rho) & =\left\{x \in \mathbb{H}^{n} \mid \max _{s \in S} d(x, \rho(s) \cdot x)<\mathrm{d}(\rho)+\varepsilon\right\} .
\end{aligned}
$$

Of course, $\mathrm{d}$ is constant on conjugacy classes, and defines a function $\mathbf{d}: m_{\Gamma}^{\prime}(n) \rightarrow \mathbb{R}_{+}$. The restriction of $\mathbf{d}$ to $m_{\Gamma}^{o}(n)$ will also be denoted by d. If $[\rho]$ is in $m_{\Gamma}^{o}(n)$, the infimum used to define $\mathrm{d}(\rho)$ is actually a minimum; to see this we use an argument of M Bestvina [4, Proposition 1.2].

Lemma 2.9 (Compare with [33, Proposition 2.5].) Let $[\rho] \in m_{\Gamma}^{o}(n)$. Then the minimum

$$
\min _{x \in \mathbb{H}^{n}} \max _{s \in S} d(x, \rho(s) \cdot x)
$$

is achieved.

Proof Consider a minimising sequence $\left(x_{k}\right)_{k \in \mathbb{N}}$ for this number. If $x_{k}$ leaves every compact subset of $\mathbb{H}^{n}$, then up to considering a subsequence, $x_{k}$ converges to a boundary point $r \in \partial \mathbb{H}^{n}$. In that case, $r$ is a global fixed point of $\rho$, and since $[\rho] \in m_{\Gamma}^{o}(n)$, there exists at least one other. Hence $\rho$ fixes (globally) a geodesic line of $\mathbb{H}^{n}$, and acts by translations on this geodesic, and then every point of this geodesic achieves the minimum. On the other hand, if $\left(x_{k}\right)_{k}$ is bounded, then it has a subsequence converging to some point $x_{\infty} \in \mathbb{H}^{n}$, which realizes this minimum.

Lemma 2.10 Let $\rho \in m_{\Gamma}^{o}(n)$. If $\rho$ fixes at least one point of the boundary, then $\min (\rho)$ is the convex hull of the fixed points of $\rho$ in $\partial \mathbb{H}^{n}$; this is a totally geodesic subspace of $\mathbb{H}^{n}$. Otherwise, $\min (\rho)$ is compact. In all cases, $\min _{\varepsilon}(\rho)$ is at bounded distance from $\min (\rho)$; that is, for all $\varepsilon>0$, there exists $k>0$ such that for all $x \in \min _{\varepsilon}(\rho)$, we have $d\left(x, \min _{\varepsilon}(\rho)\right)<k$.

Proof First suppose that $\rho$ has no fixed points in $\partial \mathbb{H}^{n}$. If $\min _{\varepsilon}(\rho)$ was unbounded for some $\varepsilon>0$, there would exist a sequence $\left(x_{k}\right)_{k \in \mathbb{N}}$ of elements of $\min _{\varepsilon}(\rho)$, converging to a point $x_{\infty} \in \partial \mathbb{H}^{n}$, and then $x_{\infty}$ would be a fixed point of $\rho$, a contradiction. Hence, for all $\varepsilon>0, \min _{\varepsilon}(\rho)$ is bounded; moreover $\min (\rho)$ is a closed subset of $\mathbb{H}^{n}$, hence compact; this finishes the proof in this case. 
Now suppose that $\rho$ has at least two distinct fixed points $x_{1}, x_{2}$ in $\partial \mathbb{H}^{n}$. If $\mathrm{d}(\rho) \neq 0$, then there exists $s \in S$ such that $\rho(s)$ is loxodromic, of axis $\left(x_{1}, x_{2}\right)$, and then $\min (\rho)=\min (\rho(s))=\left(x_{1}, x_{2}\right)$; so $\rho$ does not have any other fixed points in $\mathbb{H}^{n}$, and for all $\varepsilon>0, \min _{\varepsilon}(\rho)$ lies at bounded distance from $\min (\rho)$, by Fact 2.1. If $\mathrm{d}(\rho)=0$, then $\rho$ fixes pointwise at least the line $\left(x_{1}, x_{2}\right)$. Then

$$
\min (\rho)=\left\{x \in \mathbb{H}^{n} \mid \forall s \in S, \rho(s) \cdot x=x\right\}=\bigcap_{s \in S} \min (\rho(s))
$$

is an intersection of totally geodesic subspaces of $\mathbb{H}^{n}$, hence it is a totally geodesic subspace of $\mathbb{H}^{n}$; and it is the convex hull of the fixed points of $\rho$ in $\partial \mathbb{H}^{n}$. For all $\varepsilon>0$, we then have $\min _{\varepsilon}(\rho)=\bigcap_{s \in S} \min _{\varepsilon}(\rho(s))$. By Fact 2.1, for all $s \in S$, the set $\min _{\varepsilon}(\rho(s))$ is at bounded distance from the subspace $\min (\rho)$. One then checks by induction on $\operatorname{Card}(S)$ that $\min _{\varepsilon}(\rho)$ is at bounded distance from $\min (\rho)$.

Proposition 2.11 The function $\mathrm{d}: R_{\Gamma}(n) \rightarrow \mathbb{R}_{+}$is continuous.

Proof Suppose $\rho_{k} \rightarrow \rho$. By construction,

$$
\begin{aligned}
\{\rho \mid \mathrm{d}(\rho)<a\} & =\left\{\rho \mid \inf _{x \in \partial \mathbb{H}^{n}} \max _{s \in S} d(x, \rho(s) x)<a\right\} \\
& =\left\{\rho \mid \exists x \in \mathbb{H}^{n}, \forall s \in S, d(x, \rho(s) x)<a\right\}
\end{aligned}
$$

is open (that is, the function $\mathrm{d}$ is upper semicontinuous). For all $\varepsilon$, we thus have $\mathrm{d}\left(\rho_{k}\right)<\mathrm{d}(\rho)+\varepsilon$, for $k$ large enough. In particular $\mathrm{d}$ is continuous at every $\rho$ such that $\mathrm{d}(\rho)=0$. Hence, we suppose now $\mathrm{d}(\rho)>0$.

If $\rho$ has at least one global fixed point $x \in \partial \mathbb{H}^{n}$, then $\mathrm{d}(\rho)$ is the maximum of translation distances of the $\rho(s), s \in S$, such that $\rho(s)$ is loxodromic. Let $s_{0}$ be an element of $S$ maximising this translation distance. Then, if $\varepsilon>0$, for all $k$ large enough, $\rho_{k}\left(s_{0}\right)$ is loxodromic, and its translation distance is close to that of $\rho\left(s_{0}\right)$, so that $\mathrm{d}\left(\rho_{k}\right)>\mathrm{d}(\rho)-\varepsilon$.

Now suppose that $\rho$ is nonparabolic. Let $\varepsilon>0$. Since the topology of $\operatorname{Isom}^{+}\left(\mathbb{H}^{n}\right)$ coincides with the compact-open topology and since the set $\overline{\min _{3 \varepsilon}(\rho)}$ is compact, for all $k$ large enough, for all $x \in \overline{\min _{3 \varepsilon}(\rho)}$ and all $s \in S$, we thus have $\mid d\left(x, \rho_{k}(s) x\right)-$ $d(x, \rho(s) x) \mid<\varepsilon$. It follows that the convex function $x \mapsto \max _{s \in S} d\left(x, \rho_{k}(s) x\right)$ has a local minimum in the open set $\min _{3 \varepsilon}(\rho)$. It follows that this minimum is global, whence $\left|\mathrm{d}(\rho)-\mathrm{d}\left(\rho_{k}\right)\right| \leq 4 \varepsilon$ for $k$ large enough.

We turn to a second step towards the proof of Theorem 2.5. 
Proposition 2.12 The space $m_{\Gamma}^{o}(n)$, equipped with the induced topology, is Hausdorff.

Proof Once again, the space $m_{\Gamma}^{\prime}(n)$ being locally second countable, we can use sequences in this space. Let $\left[\rho_{1}\right],\left[\rho_{2}\right] \in m_{\Gamma}^{o}(n)$. Suppose that $\left[\rho_{1}\right]$ and $\left[\rho_{2}\right]$ cannot be separated by open sets. This means that there exists a sequence $\left(\left[\rho_{k}\right]\right)_{k} \in\left(m_{\Gamma}^{\prime}(n)\right)^{\mathbb{N}}$ converging to both $\left[\rho_{1}\right]$ and $\left[\rho_{2}\right]$, in other words, there exist $g_{k}, h_{k} \in \operatorname{SO}_{\mathbb{R}}^{+}(n, 1)$ such that $g_{k} \rho_{k} g_{k}^{-1} \rightarrow \rho_{1}$ and $h_{k} \rho_{k} h_{k}^{-1} \rightarrow \rho_{2}$. Up to conjugating $\rho_{k}$ by $h_{k}$, we may suppose that $h_{k}=1$.

First suppose $\rho_{2}$ is nonparabolic. Note $\mathrm{d}\left(\rho_{1}\right)=\mathrm{d}\left(\rho_{2}\right)$, by Proposition 2.11. Let $x \in$ $\min \left(\rho_{1}\right)$; fix $\varepsilon>0$. Then for all $\gamma \in \Gamma, d\left(g_{k} \rho_{k}(\gamma) g_{k}^{-1} x, x\right)=d\left(\rho_{k}(\gamma) g_{k}^{-1} x, g_{k}^{-1} x\right)$, hence for all $k$ large enough, $g_{k}^{-1} x \in \min _{\varepsilon}\left(\rho_{2}\right)$. Since $\min _{\varepsilon}\left(\rho_{2}\right)$ is bounded, $g_{k}$ stays in a compact set (by Fact 2.2) and hence, up to taking a subsequence, $\left(g_{k}\right)_{k}$ converges to some element $g_{\infty} \in \operatorname{Isom}^{+}\left(\mathbb{H}^{n}\right)$, and $\rho_{1}$ and $\rho_{2}$ are conjugate. Of course this argument still works after exchanging the roles of $\rho_{1}$ and $\rho_{2}$.

Now suppose that $\rho_{1}$ and $\rho_{2}$ each have at least two distinct fixed points in $\partial \mathbb{H}^{n}$. Fix $x \in \min \left(\rho_{1}\right)$. Then, as before, for all $\varepsilon>0$ and all $k$ large enough we have $g_{k}^{-1} x \in$ $\min _{\varepsilon}\left(\rho_{2}\right)$. If $d\left(x, g_{k}^{-1} x\right)$ is bounded then we can finish as in the preceding case. Let us then suppose that up to considering a subsequence, the sequence $\left(g_{k}^{-1} x\right)_{k}$ converges to a point $r_{1} \in \partial \mathbb{H}^{n}$. Then $r_{1}$ is a fixed point of $\rho_{2}$. Choose another fixed point $r_{2} \in \partial \mathbb{H}^{n}$ of $\rho_{2}$. Denote by $r_{k}$ the second end of the axis $\left(r_{2}, g_{k}^{-1} x\right)$. Then the sequence $\left(r_{k}\right)_{k}$ in $\partial \mathbb{H}^{n}$ converges to $r_{1}$. Hence there exists a sequence $\left(u_{k}\right)_{k \in \mathbb{N}}$ of elements of Isom $^{+}\left(\mathbb{H}^{n}\right)$, converging to $\operatorname{Id}_{\mathbb{H}^{n}}$ and such that $u_{k}$ fixes $r_{2}$, and sends $r_{k}$ to $r_{1}$. Let $y$ be the projection of $x$ on the axis $\left(r_{1}, r_{2}\right)$, and let $\varphi_{k}$ be the hyperbolic element of axis $\left(r_{1}, r_{2}\right)$ sending $u_{k} g_{k}^{-1} x$ to $y$. We have $\varphi_{k} u_{k} g_{k}^{-1} x=y$, the points $x$ and $y$ being fixed: it follows from Fact 2.2 that, up to extract it, the sequence $\left(\varphi_{k} u_{k} g_{k}^{-1}\right)_{k \in \mathbb{N}}$ converges to some $\varphi \in \operatorname{Isom}^{+}\left(\mathbb{H}^{n}\right)$. Now we have $\varphi_{k} u_{k} \rho_{k} u_{k}^{-1} \varphi_{k}^{-1} \rightarrow \varphi \rho_{1} \varphi^{-1}$, and $\rho_{k} \rightarrow \rho_{2}$, thus also $u_{k} \rho_{k} u_{k}^{-1} \rightarrow \rho_{2}$. Put $\rho_{k}^{\prime}=u_{k} \rho_{k} u_{k}^{-1}$, and up to conjugating everything simultaneously, suppose that the axes $\left(r_{1}, r_{2}\right)$ and $(0, \infty)$ coincide. Since $\rho_{2}$ and $\varphi_{k}$ preserve that axis, for every $\gamma \in \Gamma$ we can write the elements $\rho_{2}(\gamma), \rho_{k}^{\prime}(\gamma)$ and $\varphi_{k}$ in the form

$$
\rho_{2}(\gamma)=\left(\begin{array}{ccc}
\lambda(\gamma) & 0 & (0) \\
0 & 1 / \lambda(\gamma) & (0) \\
(0) & (0) & A(\gamma)
\end{array}\right), \quad \rho_{k}^{\prime}(\gamma)=\left(\begin{array}{ccc}
a_{k}(\gamma) & b_{k}(\gamma) & X_{k}(\gamma) \\
c_{k}(\gamma) & d_{k}(\gamma) & Y_{k}(\gamma) \\
Z_{k}(\gamma) & W_{k}(\gamma) & A_{k}(\gamma)
\end{array}\right)
$$

and

$$
\varphi_{k}=\left(\begin{array}{ccc}
t_{k} & & (0) \\
& 1 / t_{k} & \\
(0) & & I_{n-1}
\end{array}\right)
$$


where $\lim _{k \rightarrow+\infty} t_{k}=+\infty$, up to conjugating in order to exchange the points 0 and $\infty$ in $\partial \mathbb{H}^{n}$. Then

so that $\quad \varphi \rho_{1}(\gamma) \varphi^{-1}=\left(\begin{array}{ccc}\lambda(\gamma) & 0 & (0) \\ r(\gamma) & 1 / \lambda(\gamma) & Y(\gamma) \\ Z(\gamma) & (0) & A(\gamma)\end{array}\right)$

Since $\left[\rho_{1}\right] \in m_{\Gamma}^{o}(n)$, the representation $\varphi \rho_{1} \varphi^{-1}$ fixes another point of $\partial \mathbb{H}^{n}$ than 0 ; denote it by $r_{3}$. There exists an isometry $\phi \in \operatorname{Isom}^{+}\left(\mathbb{H}^{n}\right)$ fixing 0 and sending $r_{3}$ to $\infty$, and now $\phi \varphi \rho_{1} \varphi^{-1} \phi^{-1}$ and $\rho_{2}$ are conjugate.

Now we prove another step towards Theorem 2.5:

Proposition 2.13 On the set $m_{\Gamma}^{o}(n)$, the induced topology defined by $m_{\Gamma}^{o}(n) \hookrightarrow$ $m_{\Gamma}^{\prime}(n)$ and the final topology defined by $\pi: m_{\Gamma}^{\prime}(n) \rightarrow m_{\Gamma}^{o}(n)$ coincide (in particular, $\pi$ is continuous).

Proof Let $U$ be an open subset of $m_{\Gamma}^{o}(n)$ for the final topology. Then $\pi^{-1}(U)$ is open in $m_{\Gamma}^{\prime}(n)$, hence the set $m_{\Gamma}^{o}(n) \cap \pi^{-1}(U)$ is open for the induced topology. But $m_{\Gamma}^{o}(n) \cap \pi^{-1}(U)=U$, since $\pi$ is the identity on $m_{\Gamma}^{o}(n)$. Consequently, in order to prove the proposition it suffices to prove that $\pi$ is continuous. Once again, $m_{\Gamma}^{\prime}(n)$ being locally second countable, we can use a sequential criterium.

Suppose that $\rho_{k}, \rho \in R_{\Gamma}(n)$ and $\rho_{k} \rightarrow \rho$. We want to prove that $\pi([\rho(k)])$ converges to $\pi([\rho])$. The elements $\rho_{k}$ have either zero, or at least one fixed point in $\partial \mathbb{H}^{n}$. Up to consider two distinct subsequences, we may suppose that this situation does not depend on $k$. If $\rho_{k}$ is nonparabolic, for all $k$, then $\pi\left(\left[\rho_{k}\right]\right)=\left[\rho_{k}\right] \rightarrow[\rho]$. Since every neighborhood of $\pi([\rho])$ contains $[\rho]$, the sequence $\pi\left(\left[\rho_{k}\right]\right)$ also converges to $\pi([\rho])$. Suppose finally that $\rho_{k}$ has at least one fixed point in $\partial \mathbb{H}^{n}$, for all $k \in \mathbb{N}$. By Lemma 2.7, the representation $\rho$ has at least one fixed point in $\partial \mathbb{H}^{n}$, and as before, $\left[\rho_{k}\right]$ also converges to $\pi([\rho])$. Once again, $\rho_{k}$ has either at least two, or exactly one fixed point in $\partial \mathbb{H}^{n}$, and up to considering two subsequences, we may suppose that this does not depend on $k$. In the first case, we have $\pi\left(\left[\rho_{k}\right]\right)=\left[\rho_{k}\right]$ so $\pi\left(\left[\rho_{k}\right]\right) \rightarrow \pi([\rho])$. In the second case, Lemma 2.8 says that $\pi\left(\left[\rho_{k}\right]\right)$ converges to $\pi([\rho])$.

Now that we proved that these two topologies coincide, we will equip the set $m_{\Gamma}^{o}(n)$ with this topology in the sequel, without having to precise which topology we consider. Now we finish the proof of Theorem 2.5: 
Corollary 2.14 The map d: $m_{\Gamma}^{o}(n) \rightarrow \mathbb{R}_{+}$is continuous, and for all $A>0$, the preimage $\mathbf{d}^{-1}([0, A])$ is compact. In particular, the space $m_{\Gamma}^{o}(n)$ is locally compact.

Proof The continuous map d: $R_{\Gamma}(n) \rightarrow \mathbb{R}_{+}$is constant on the fibres of the map $R_{\Gamma}(n) \rightarrow m_{\Gamma}^{o}(n)$, hence $\mathbf{d}$ is continuous, by considering the final topology on $m_{\Gamma}^{o}(n)$. Now, let $A>0$; let us prove that $\mathbf{d}^{-1}([0, A])$ is compact. Fix $x_{0} \in \mathbb{H}^{n}$, and denote by $R_{\Gamma}^{A} \subset R_{\Gamma}(n)$ the set of representations $\rho$ satisfying

$$
\max _{s \in S} d\left(x_{0}, \rho(s) x_{0}\right) \leq A .
$$

By Fact 2.2, this set $R_{\Gamma}^{A}$ is compact. The projection $p: R_{\Gamma}(n) \rightarrow m_{\Gamma}^{o}(n)$ is continuous, and takes values in a Hausdorff space, hence $p\left(R_{\Gamma}^{A}\right)$ is compact; it therefore suffices to check that $p\left(R_{\Gamma}^{A}\right)=\mathbf{d}^{-1}([0, A])$.

Let $[\rho] \in \mathbf{d}^{-1}([0, A])$. Then, by Lemma 2.9, there exists a point $x \in \mathbb{H}^{n}$ such that $\max _{s \in S}(x, \rho(s) x)=\mathrm{d}(\rho)$, and up to conjugating $\rho$ we may take $x=x_{0}$. We then have $\rho \in R_{\Gamma}^{A}$, so that $[\rho] \in p\left(R_{\Gamma}^{A}\right)$. Now let $\rho \in R_{\Gamma}^{A}$. By definition of $\mathrm{d}(\rho)$, we have $\mathrm{d}(\rho) \leq A$, hence $\mathbf{d}([\rho])=\mathrm{d}(\rho) \leq A$, and $p(\rho) \in \mathbf{d}^{-1}([0, A])$. Thus $p\left(R_{\Gamma}^{A}\right)=\mathbf{d}^{-1}([0, A])$.

Since the space $\mathbb{R}_{+}$is locally compact, so is $m_{\Gamma}^{o}(n)$.

The group Isom $\left(\mathbb{H}^{n}\right)$ of isometries which may not be orientation-preserving acts on $m_{\Gamma}^{o}(n)$ by conjugation. We denote by $m_{\Gamma}^{u}(n)$ the quotient of $m_{\Gamma}^{o}(n)$ by this action (" $u$ " standing for "unoriented").

In the case $n=2$, we shall prove here that this quotient is identified to the space $X_{\Gamma}(2)$ formed by characters of representations. First, let us set up some notation. From now on, if

$$
A=\left(\begin{array}{ll}
a & b \\
c & d
\end{array}\right) \in \operatorname{SL}(2, \mathbb{R})
$$

we will still denote by

$$
\left(\begin{array}{ll}
a & b \\
c & d
\end{array}\right)
$$

the corresponding element $[A] \in \operatorname{PSL}(2, \mathbb{R})$, without the symbol \pm . Following [25], we denote $\operatorname{tr}(A)=a+d$ and $\operatorname{Tr}([A])=|a+d|$. If $\rho \in R_{\Gamma}=\operatorname{Hom}(\Gamma, \operatorname{PSL}(2, \mathbb{R}))$, denote by $\chi(\rho): \Gamma \rightarrow \mathbb{R}_{+}$its character, defined by $\chi(\rho)(\gamma)=\operatorname{Tr}(\rho(\gamma))$. Note that the groups $\operatorname{PSL}(2, \mathbb{R})$ and $\mathrm{SO}_{\mathbb{R}}^{+}(2,1)$ are isomorphic, being the groups of orientation preserving isometries of two models of $\mathbb{H}^{2}$. An easy way of writing down such an isomorphism $\varphi: \operatorname{PSL}(2, \mathbb{R}) \rightarrow \mathrm{SO}_{\mathbb{R}}^{+}(2,1)$ is to consider the adjoint representation $\operatorname{SL}(2, \mathbb{R}) \rightarrow \operatorname{GL}(3, \mathbb{R})$, which has kernel $\{ \pm \mathrm{Id}\}$ and preserves the Killing form of signature $(2,1)$. A straightforward computation then gives, for all $[A] \in \operatorname{PSL}(2, \mathbb{R})$, 
$\operatorname{tr}(\varphi([A]))=\operatorname{Tr}([A])^{2}-1$. In particular, up to composition with a simple function, $\chi(\rho)$ is indeed the character of a linear representation of $\Gamma$.

Proposition 2.15 Let $\left[\rho_{1}\right],\left[\rho_{2}\right] \in m_{\Gamma}^{o}(2)$. Then $\chi\left(\rho_{1}\right)=\chi\left(\rho_{2}\right)$ if and only if there exists an isometry $u$ of $\mathbb{H}^{2}$ such that $\rho_{1}=u \rho_{2} u^{-1}$.

Proof Of course, the character is a conjugation invariant in $\operatorname{Hom}(\Gamma, \operatorname{PSL}(2, \mathbb{R}))$; hence there is only one direction to prove. The proof given here is inspired by the proof of Proposition 1.5.2 of Culler and Shalen [9].

It is easy to check that elementary subgroups of $\operatorname{PSL}(2, \mathbb{R})$ either have a global fixed point in $\mathbb{H}^{2} \cup \partial \mathbb{H}^{2}$, or are "dihedral", fixing globally a pair $\{x, y\} \subset \partial \mathbb{H}^{2}$, with a hyperbolic element of axis $(x, y)$, and an order 2 elliptic element exchanging $x$ and $y$. In a subgroup of $\operatorname{PSL}(2, \mathbb{R})$ with a fixed point in $\mathbb{H}^{2} \cup \partial \mathbb{H}^{2}$, the trace of any commutator is 2 . In a dihedral subgroup of $\operatorname{PSL}(2, \mathbb{R})$, there exists a hyperbolic commutator, but the trace of any commutator of commutators is 2 . In a nonelementary subgroup of $\operatorname{PSL}(2, \mathbb{R})$, we can use ping-pong to find a Schottky subgroup, so commutators of commutators can have different traces. In particular, the character of any representation determines if it has a fixed point, or if it is dihedral, or nonelementary.

Suppose first that $\rho_{1}$ and $\rho_{2}$ are not elementary. Then there exists $\gamma_{0} \in \Gamma$ such that $\rho_{1}\left(\gamma_{0}\right)$ is hyperbolic; $\rho_{2}\left(\gamma_{0}\right)$ is hyperbolic too, and up to conjugating $\rho_{1}$ and $\rho_{2}$ by an element of $\operatorname{PSL}(2, \mathbb{R})$, we have

$$
\rho_{1}\left(\gamma_{0}\right)=\rho_{2}\left(\gamma_{0}\right)=\left(\begin{array}{ll}
\lambda & 0 \\
0 & \frac{1}{\lambda}
\end{array}\right)
$$

with $\lambda>1$. Since $\rho_{1}$ is not elementary, there exists $\gamma_{1} \in \Gamma$ such that $\rho_{1}\left(\gamma_{1}\right)$ is hyperbolic and has no fixed points in common with $\rho_{1}\left(\gamma_{0}\right)$. Denote

$$
\rho_{1}\left(\gamma_{1}\right)=\left(\begin{array}{ll}
a & b \\
c & d
\end{array}\right) \quad \text { and } \quad \rho_{2}\left(\gamma_{1}\right)=\left(\begin{array}{ll}
a^{\prime} & b^{\prime} \\
c^{\prime} & d^{\prime}
\end{array}\right)
$$

Then for all $n \in \mathbb{Z},\left|a \lambda^{n}+d / \lambda^{n}\right|=\left|a^{\prime} \lambda^{n}+d^{\prime} / \lambda^{n}\right|$, hence, up to changing the signs of $a^{\prime}, b^{\prime}, c^{\prime}$ and $d^{\prime}$ we have $a=a^{\prime}$ and $d=d^{\prime}$. Up to conjugating by diagonal matrices, we may also suppose that $|b|=\left|b^{\prime}\right|=1$, since $\gamma_{0}$ and $\gamma_{1}$ do not share any fixed points. Up to conjugating by the reflection of axis $(0, \infty)$, we can further suppose that $b=b^{\prime}=1$, and then $c=c^{\prime}$.

Now consider any $\gamma \in \Gamma$ and denote

$$
\rho_{1}(\gamma)=\left(\begin{array}{ll}
x & y \\
z & t
\end{array}\right) \quad \text { and } \quad \rho_{2}(\gamma)=\left(\begin{array}{cc}
x^{\prime} & y^{\prime} \\
z^{\prime} & t^{\prime}
\end{array}\right) .
$$


Here again, up to simultaneously changing the signs of $x^{\prime}, y^{\prime}, z^{\prime}$ and $t^{\prime}$, we have $x=x^{\prime}$ and $t=t^{\prime}$. Now, for $m, n \in \mathbb{Z}$ the equality $\operatorname{Tr}\left(\rho_{1}\left(\gamma_{0}^{n} \gamma_{1} \gamma_{0}^{m} \gamma\right)\right)=\operatorname{Tr}\left(\rho_{2}\left(\gamma_{0}^{n} \gamma_{1} \gamma_{0}^{m} \gamma\right)\right)$ yields

$$
\begin{aligned}
\left|a x \lambda^{n+m}+z \lambda^{n-m}+c y \lambda^{m-n}+d t \lambda^{-n-m}\right| & \\
& =\left|a x \lambda^{n+m}+z^{\prime} \lambda^{n-m}+c y^{\prime} \lambda^{m-n}+d t \lambda^{-n-m}\right| .
\end{aligned}
$$

By taking $n+m=0$ and $n-m$ large, this implies $|z|=\left|z^{\prime}\right|,|y|=\left|y^{\prime}\right|$ and $z z^{\prime}$ and $y y^{\prime}$ must have the same sign. By taking $m=0$ and $n$ large, it implies $|a x+z|=\left|a x+z^{\prime}\right|$ and $|c y+d t|=\left|c y^{\prime}+d t\right|$. Since $\rho_{1}\left(\gamma_{1}\right)$ is hyperbolic, we have $a \neq 0$ or $d \neq 0$; hence $z=z^{\prime}$ and $y=y^{\prime}$. This finishes the proof, in the "generic" case.

If $\rho_{1}$ and $\rho_{2}$ are dihedral, then as before, we can find $\gamma_{0}, \gamma_{1} \in \Gamma$ such that

and

$$
\begin{aligned}
& \rho_{1}\left(\gamma_{0}\right)=\rho_{2}\left(\gamma_{0}\right)=\left(\begin{array}{cc}
\lambda & 0 \\
0 & 1 / \lambda
\end{array}\right) \quad \text { with } \lambda>1, \\
& \rho_{1}\left(\gamma_{1}\right)=\rho_{2}\left(\gamma_{1}\right)=\left(\begin{array}{cc}
0 & 1 \\
-1 & 0
\end{array}\right) .
\end{aligned}
$$

For all $\gamma \in \Gamma, \rho_{i}(\gamma)$ is then entirely determined by the absolute values of the traces of $\rho_{i}\left(\gamma_{0}^{n} \gamma\right)$ and $\rho_{i}\left(\gamma_{0}^{n} \gamma_{1} \gamma\right)$.

If $\rho_{1}$ and $\rho_{2}$ have at least one global fixed point in $\partial \mathbb{H}^{2}$, then they have two, hence all the elements $\rho_{i}(\gamma)$ are hyperbolic and share the same axis; $\rho_{i}$ then identifies to an action of $\Gamma$ on an axis $\mathbb{R}$ by translations, which is determined by its character. Finally, if $\rho_{1}$ possesses a global fixed point in $\mathbb{H}^{2}$, then so does $\rho_{2}$. Hence, up to conjugating $\rho_{1}$ and $\rho_{2}$, there exist morphisms $\varphi_{i}: \Gamma \rightarrow \mathbb{R}$ such that for all $\gamma \in \Gamma$,

$$
\rho_{i}(\gamma)=\left(\begin{array}{cc}
\cos \varphi_{i}(\gamma) & -\sin \varphi_{i}(\gamma) \\
\sin \varphi_{i}(\gamma) & \cos \varphi_{i}(\gamma)
\end{array}\right)
$$

with $\left|\cos \varphi_{1}\right|=\left|\cos \varphi_{2}\right|$. We can check again that $\rho_{1}$ and $\rho_{2}$ are conjugated by an isometry.

The two quotients $m_{\Gamma}^{u}(2)$ and $X_{\Gamma}(2)$ of the space $R_{\Gamma}(2)$ are therefore identical. Hence, $m_{\Gamma}^{o}(2)$ is just an oriented version of the space of characters.

\subsection{A reminder of the Bestvina-Paulin compactification of $m_{g}^{u}(n)$}

2.2.1 The equivariant Gromov topology We are now going to recall F Paulin's construction of the compactification of representation spaces, in order to adapt it to the whole space $m_{\Gamma}^{u}(n)$. We refer to [37] for an efficient exposition of this construction. 
Here we will be interested in metric spaces $(X, d)$, equipped with actions of $\Gamma$ by isometries, ie, morphisms $\rho: \Gamma \rightarrow \operatorname{Isom}(X, d)$; we say that $(\rho, X, d)$ is equivalent to $\left(\rho^{\prime}, X^{\prime}, d^{\prime}\right)$ if there exists a $\Gamma$-equivariant isometry between $X$ and $X^{\prime}$. Let $\mathcal{E}$ be a set of classes of actions of $\Gamma$ on metric spaces up to equivariant isometries. If $(\rho, X, d), \quad\left(\rho^{\prime}, X^{\prime}, d^{\prime}\right) \in \mathcal{E}$ (in order to avoid too heavy notation, we denote again by $(\rho, X, d)$ and $\left(\rho^{\prime}, X^{\prime}, d^{\prime}\right)$ their classes under equivariant isometry), if $\varepsilon>0$, if $K=\left(x_{1}, \ldots, x_{p}\right)$ and $K^{\prime}=\left(x_{1}^{\prime}, \ldots, x_{p}^{\prime}\right)$ are finite sequences (of the same length) in $X$ and $X^{\prime}$, and if $P$ is a finite subset of $\Gamma$, we say that $K^{\prime}$ is a $P$-equivariant $\varepsilon$-approximation of $K$ if for all $g, h \in P$, and all $i, j \in\{1, \ldots, p\}$, we have

$$
\left|d\left(\rho(g) \cdot x_{i}, \rho(h) \cdot x_{j}\right)-d^{\prime}\left(\rho^{\prime}(g) \cdot x_{i}^{\prime}, \rho^{\prime}(h) \cdot x_{j}^{\prime}\right)\right|<\varepsilon .
$$

Given $(\rho, X, d) \in \mathcal{E}$ and $K, \varepsilon$ and $P$ as above, we define $U_{K, \varepsilon, P}(\rho, X, d)$ as the subset of $\mathcal{E}$ consisting of those $\left(\rho^{\prime}, X^{\prime}, d^{\prime}\right)$ such that $X^{\prime}$ contains a $P$-equivariant $\varepsilon-$ approximation of $K$. The sets $U_{K, \varepsilon, P}(\rho, X, d)$ form a basis of open sets of a topology, called the equivariant Gromov topology (see [35; 21]).

By definition, every representation $\rho \in R_{\Gamma}(n)$ defines an action of $\Gamma$ by isometries on the metric space $\left(\mathbb{H}^{n}, d_{\mathbb{H}^{n}}\right.$ ) (where $d_{\mathbb{H}^{n}}$ is the usual distance on $\mathbb{H}^{n}$ ), and every conjugation by an isometry of $\mathbb{H}^{n}$ defines an equivariant isometry. Hence, every element $[\rho] \in m_{\Gamma}^{u}(n)$ defines a unique equivariant isometry class of actions $\left(\rho, \mathbb{H}^{n}, d_{\mathbb{H}^{n}}\right)$; we can therefore consider the set $m_{\Gamma}^{u}(n)$ as a set of (equivariant isometry classes of) actions of $\Gamma$ on $\left(\mathbb{H}^{n}, d_{\mathbb{H}^{n}}\right)$ and we can equip this set with the equivariant Gromov topology.

Proposition 2.16 (F Paulin [35, Proposition 6.2]) On the set $m_{\Gamma}^{u}(n)$, the usual topology and the equivariant Gromov topology coincide.

Since we will have to adapt this proposition to the oriented case when $n=2$, we recall here the proof given in [35], in that case. Note, anyway, that the general case $n \geq 2$ is proved similarly.

Proof in the case $n=2$ The usual topology is of course finer than the equivariant Gromov topology, as the distances considered are continuous for the usual topology on $m_{\Gamma}^{u}$. Conversely, fix $\varepsilon>0$, and let $\left[\rho_{k}\right]$ be a sequence converging to $\left[\rho_{\infty}\right]$ for the equivariant Gromov topology. Let us prove that up to conjugating these representations, $\rho_{k} \rightarrow \rho_{\infty}$ (since $\mathbb{H}^{2}$ is separable, the space $m_{\Gamma}^{u}$, equipped with the equivariant Gromov topology, is locally second countable; hence we can indeed use sequences in that space). Consider three points $x_{1}, x_{2}, x_{3} \in \mathbb{H}^{2}$ which form a nondegenerate triangle. Then, for all $\varepsilon^{\prime}>0$, and for $k$ large enough, there exists a triple $\left(x_{1}^{k}, x_{2}^{k}, x_{3}^{k}\right) \in \mathbb{H}^{2}$ such that for every $i, j \in\{1,2,3\}$ and every $s_{1}, s_{2} \in S$,

$$
\left|d\left(\rho_{\infty}\left(s_{1}\right) x_{i}, \rho_{\infty}\left(s_{2}\right) x_{j}\right)-d\left(\rho_{k}\left(s_{1}\right) x_{i}^{k}, \rho_{k}\left(s_{2}\right) x_{j}^{k}\right)\right|<\varepsilon .
$$


Now let $S=\left\{s_{1}, \ldots, s_{n}\right\}$ and $y_{1}=\rho_{\infty}\left(s_{1}\right) x_{1}, y_{2}=\rho_{\infty}\left(s_{1}\right) x_{2}, \ldots, y_{3 n}=\rho_{\infty}\left(s_{n}\right) x_{3}$, and similarly define $y_{1}^{k}, \ldots, y_{3 n}^{k} \in \mathbb{H}^{2}$. The following fact will enable us to conclude:

Fact 2.17 For all $\varepsilon>0$, there exists $\varepsilon^{\prime}>0$ such that for all $x_{1}, \ldots, x_{n}, x_{1}^{\prime}, \ldots, x_{n}^{\prime}$ in $\mathbb{H}^{2}$, if for all $i, j,\left|d\left(x_{i}, x_{j}\right)-d\left(x_{i}^{\prime}, x_{j}^{\prime}\right)\right|<\varepsilon^{\prime}$ then there exists an isometry $\varphi$ of $\mathbb{H}^{2}$ such that $d\left(\varphi\left(x_{i}\right), x_{i}^{\prime}\right)<\varepsilon$.

This is left as an exercise (see eg [43, Proposition 1.1.8]) and follows from the fact that the sine and cosine laws, in the hyperbolic plane, can be used to recover continuously a triangle from its three lengths. In particular, up to conjugating $\rho_{k}$ by an isometry of $\mathbb{H}^{2}$, we have $d\left(y_{i}, y_{i}^{k}\right)<\varepsilon$, and for every $s \in S$, it follows that $d\left(x_{i}, \rho_{\infty}(s)^{-1} \cdot \rho_{k}(s) x_{i}\right)<\varepsilon$, for the three nonaligned points $x_{1}, x_{2}, x_{3}$, so that $\rho_{k} \rightarrow \rho_{\infty}$ in the usual topology.

For all $\rho \in m_{\Gamma}^{u}(n)$, define

$$
\ell(\rho)=\max (1, \mathrm{~d}(\rho))
$$

and equip the set $\mathbb{H}^{n}$ with the distance $d_{\mathbb{H}^{n}} / \ell(\rho)$. From now on, every element $[\rho] \in m_{\Gamma}^{u}(n)$ will be associated to $\left(\rho, \mathbb{H}^{n}, d_{\mathbb{H}^{n}} / \ell(\rho)\right)$ instead of $\left(\rho, \mathbb{H}^{n}, d_{\mathbb{H}^{n}}\right)$ (this is another realization of $m_{\Gamma}^{u}(n)$ as a set of (classes of) actions of $\Gamma$ on $\mathbb{H}^{n}$, and the equivariant Gromov topology is still the same, by Proposition 2.16 and Corollary 2.14).

As such, the equivariant Gromov topology does not separate any action on a space from the restricted actions on invariant subspaces. In particular, if we consider the whole space $m_{\Gamma}^{u}(n)$ as well as actions of $\Gamma$ on $\mathbb{R}$-trees, including actions on lines, this will yield a non-Hausdorff space, since some of the elements of $m_{\Gamma}^{u}(n)$ have an invariant line. In order to get rid of this little degeneracy, we are going to modify slightly the definition of the equivariant Gromov topology, so that elementary representations in $m_{\Gamma}^{u}$ will be separated from the corresponding actions on lines, when considered as actions on $\mathbb{R}$-trees. If $\mathcal{E}$ is a set of (classes of) actions of $\Gamma$ by isometries on spaces which are hyperbolic in the sense of Gromov, put $U_{K, \varepsilon, P}^{\prime}(\rho, X, d)$ to be the subset of $\mathcal{E}$ consisting of those $\left(\rho^{\prime}, X^{\prime}, d^{\prime}\right)$ such that there exist $x_{1}^{\prime}, \ldots, x_{p}^{\prime} \in X^{\prime}$, such that for all $g, h \in P$, and all $i, j \in\{1, \ldots, p\}$, we have

$$
\left|d\left(\rho(g) \cdot x_{i}, \rho(h) \cdot x_{j}\right)-d^{\prime}\left(\rho^{\prime}(g) \cdot x_{i}^{\prime}, \rho^{\prime}(h) \cdot x_{j}^{\prime}\right)\right|<\varepsilon \quad \text { and } \quad\left|\delta(X)-\delta\left(X^{\prime}\right)\right|<\varepsilon \text {. }
$$

By $\delta(X)$, we mean the lowest constant such that $X$ is Gromov $\delta(X)$-hyperbolic.

As we shall see very soon (Proposition 2.18), this extra condition changes the equivariant Gromov topology only at the neighborhood of elementary representations. It is comparable to the fact of adding 2 to characters, as it is done by J Morgan and P Shalen in [32], in order not to bother with the neighborhood of the trivial representation. 
2.2.2 The space $\overline{m_{\Gamma}^{u}(n)}$ Let us first recall that if $X$ is a topological space, a compactification of $X$ is a couple $(\bar{X}, i)$ such that $\bar{X}$ is a compact Hausdorff space, $i: X \hookrightarrow \bar{X}$ is a homeomorphism on its image, and such that $i(X)$ is open and dense in $\bar{X}$.

Note that we request $\bar{X}$ to be Hausdorff. In particular, a space $X$ needs to be locally compact in order to admit a compactification. If $X$ is a locally compact space and $(\bar{X}, i)$ is a compactification of $X$, then the compact set $\bar{X} \backslash X$ is called the boundary of $X$, and is denoted by $\partial \bar{X}$, or $\partial X$ if no confusion is possible between different compactifications of $X$. The points in the boundary are called the ideal points of the compactification.

Note that if $X$ is locally compact and if $(\bar{X}, i)$ is a compactification of $X$, then the open subsets of $\bar{X}$ containing $\partial \bar{X}$ are precisely the complements, in $\bar{X}$, of the compact subsets of $X$.

Finally, if $X$ is a locally compact space, if $Y$ is Hausdorff and $f: X \rightarrow Y$ is continuous and has a relatively compact image, then we can define a compactification of $X$ as follows (see [32, page 415]). Denote by $\widehat{X}=X \cup\{\infty\}$ the Alexandrov compactification of $X$ (the one-point compactification), we define $i: X \rightarrow \hat{X} \times Y$ by $i(x)=(x, f(x))$. Denote by $\bar{X}$ the closure of $i(X)$ in $\hat{X} \times Y$. Then $(\bar{X}, i)$ is a compactification of $X$; we say that it is the compactification defined by $f$.

We say that an action of $\Gamma$ on an $\mathbb{R}$-tree $T$ is minimal if $T$ has no proper invariant subtree. The equivalence classes of $\mathbb{R}$-trees equipped with minimal actions of $\Gamma$ by isometries, up to equivariant isometry, form a set, and we denote by $\mathcal{T}^{\prime}(\Gamma)$ the subset formed by trees not reduced to a point. In order to exhibit this set, one can prove that the $\mathbb{R}$-tree $T$ and the action of $\Gamma$ are entirely determined by the set $\{d(p, \gamma \cdot p) \mid \gamma \in \Gamma\}$ (see $[1 ; 32])$.

We have modified the definition of the equivariant Gromov topology so that we would be considering Hausdorff spaces, and for this reason we are also going to restrict the set of $\mathbb{R}$-trees we consider. If $(\rho, T)$ possesses an end which is globally fixed by $\rho$ (we then say that this action is reducible; see eg [36]), then $(\rho, T, d)$ is not separated, in the equivariant Gromov topology, from the action on a line which has the same translation lengths. Therefore, we shall restrict ourselves to the subset $\mathcal{T}(\Gamma) \subset \mathcal{T}^{\prime}(\Gamma)$ consisting of actions on lines and actions on $\mathbb{R}$-trees without fixed ends, such that $\min _{x_{0} \in T} \max _{\gamma \in S} d\left(x_{0}, \gamma \cdot x_{0}\right)=1$.

In the sequel, we equip the set $m_{\Gamma}^{u}(n) \cup \mathcal{T}(\Gamma)$ with the modified equivariant Gromov topology, and we let $\overline{m_{\Gamma}^{u}(n)}$ denote the closure of the set $m_{\Gamma}^{u}(n)$ in the space $m_{\Gamma}^{u}(n) \cup \mathcal{T}(\Gamma)$. 
The following proposition is simply a detailed version of an argument of F Paulin [35] saying that $\mathbb{R}$-trees and hyperbolic structures are not indistinguishable by the equivariant Gromov topology, so that the modified equivariant Gromov topology changes it only at the boundary of degenerate representations.

In this section, we say that a finite sequence $K$ is big if it contains four points $A, B_{1}, B_{2}, B_{3}$ such that $d\left(A, B_{i}\right)=1, d\left(B_{1}, B_{3}\right)=2$ and $d\left(B_{1}, B_{2}\right)=d\left(B_{2}, B_{3}\right)$. In an $\mathbb{R}$-tree, this means that their convex hull is a tripod of centre $A$, and this implies that $d\left(B_{1}, B_{2}\right)=2$. In $\mathbb{H}^{n}$, this means that the segments $\left[B_{1}, B_{3}\right]$ and $\left[B_{2}, A\right]$ meet orthogonally at $A$.

Proposition 2.18 Let $(\rho, X, d) \in m_{\Gamma}^{u}(n) \cup \mathcal{T}(\Gamma)$ and let $K \subset X$ be a big finite sequence. Let $\varepsilon>0$. Then for all $\varepsilon^{\prime}>0$ small enough, for all $\left(\rho^{\prime}, X^{\prime}, d^{\prime}\right) \in$ $U_{K, \varepsilon^{\prime},\{1\}}(\rho, X, d)$, we have $\left|\delta\left(X^{\prime}\right)-\delta(X)\right|<\varepsilon$.

Here, by $\varepsilon^{\prime}$ small enough, we mean: $\varepsilon^{\prime}<v(\delta(X), \varepsilon)$, where $v: \mathbb{R}_{+} \times \mathbb{R}_{+}^{*} \rightarrow \mathbb{R}_{+}^{*}$ is some (universal) function.

Proof Let $\varepsilon^{\prime}>0$, and let $K^{\prime}=\left(A^{\prime}, B_{1}^{\prime}, B_{2}^{\prime}, B_{3}^{\prime}\right) \subset X^{\prime}$ be an $\varepsilon^{\prime}$-approximation of $K$. Put $B_{1}^{\prime \prime}=B_{1}^{\prime}$. We have $\left|d\left(B_{1}^{\prime \prime}, B_{3}^{\prime}\right)-2\right|<\varepsilon^{\prime}$ so we can choose a point $B_{3}^{\prime \prime} \in X^{\prime}$ such that $d\left(B_{3}^{\prime}, B_{3}^{\prime \prime}\right)<\varepsilon^{\prime}$ and $d\left(B_{1}^{\prime \prime}, B_{3}^{\prime \prime}\right)=2$. Denote by $r(t)$ the geodesic segment, in $X^{\prime}$, such that $r(0)=B_{1}^{\prime \prime}$ and $r(2)=B_{3}^{\prime \prime}$, and put $A^{\prime \prime}=r(1)$. Then the CAT(0) property on $X^{\prime}$ implies that $d\left(A^{\prime}, A^{\prime \prime}\right)<\sqrt{2 \varepsilon^{\prime}+\varepsilon^{\prime 2}}$. Finally, we have $\left|d\left(A^{\prime}, B_{2}^{\prime}\right)-1\right|<\varepsilon^{\prime}$ so there exists $B_{2}^{\prime \prime} \in X$ such that $d\left(B_{2}^{\prime}, B_{2}^{\prime \prime}\right)<\varepsilon^{\prime}+\sqrt{\frac{2 \varepsilon^{\prime}+\varepsilon^{\prime 2}}{2 \varepsilon^{\prime}+\varepsilon^{\prime 2}}}$ and $d\left(A^{\prime \prime}, B_{2}^{\prime \prime}\right)=1$. We also have the inequalities $\left|d\left(B_{1}^{\prime \prime}, B_{2}^{\prime \prime}\right)-1\right|<2 \varepsilon^{\prime}+\sqrt{2 \varepsilon^{\prime}+\varepsilon^{\prime 2}}$, and $\left|d\left(B_{3}^{\prime \prime}, B_{2}^{\prime \prime}\right)-d\left(B_{1}^{\prime \prime}, B_{2}^{\prime \prime}\right)\right|<5 \varepsilon^{\prime}+2 \sqrt{2 \varepsilon^{\prime}+\varepsilon^{\prime 2}}$.

First suppose that $\delta(X) \neq 0$, and denote $x=\delta_{\mathbb{H}^{2}} / \delta(X)$. The cosine law $\mathrm{I}$, in $\left(\mathbb{H}^{2}, d_{\mathbb{H}^{2}}\right)$, implies that $\cosh \left(x d\left(B_{1}, B_{2}\right)\right)=\cosh ^{2}(x)$. For all $\varepsilon^{\prime}$ small enough, we have $d\left(A^{\prime \prime}, B_{i}^{\prime \prime}\right)=1, d\left(B_{1}^{\prime \prime}, B_{3}^{\prime \prime}\right)=2, d\left(B_{1}^{\prime \prime}, B_{2}^{\prime \prime}\right)<2$ and $d\left(B_{3}^{\prime \prime}, B_{2}^{\prime \prime}\right)<2$, so that $X^{\prime}$ cannot be an $\mathbb{R}$-tree. Put also $x^{\prime}=\delta_{\mathbb{H}^{2}} / \delta\left(X^{\prime}\right)$. Then the cosine law I in $X^{\prime}$ implies that $\cosh \left(x^{\prime} d\left(B_{1}^{\prime \prime}, B_{2}^{\prime \prime}\right)\right)+\cosh \left(x^{\prime} d\left(B_{2}^{\prime \prime}, B_{3}^{\prime \prime}\right)\right)=2 \cosh ^{2}\left(x^{\prime}\right)$. It follows from the study of the function $F:[0,2] \times \mathbb{R}_{+} \rightarrow \mathbb{R}$ defined by $F(b, x)=\cosh ^{2}(x)-\cosh (x b)$, that by taking $\varepsilon^{\prime}$ small enough we can force $x$ and $x^{\prime}$ to be arbitrarily close.

Now suppose that $\delta(X)=0$. If $\delta\left(X^{\prime}\right)=0$ then there is nothing to do. Otherwise, we have again $\cosh \left(x^{\prime} d\left(B_{1}^{\prime \prime}, B_{2}^{\prime \prime}\right)\right)+\cosh \left(x^{\prime} d\left(B_{2}^{\prime \prime}, B_{3}^{\prime \prime}\right)\right)=2 \cosh ^{2}\left(x^{\prime}\right)$, where, for $\varepsilon^{\prime}$ small enough, the distances $d\left(B_{1}^{\prime \prime}, B_{2}^{\prime \prime}\right)$ and $d\left(B_{2}^{\prime \prime}, B_{3}^{\prime \prime}\right)$ can be taken arbitrarily close to 2 , which implies that $x^{\prime}$ can be forced to be arbitrarily large. 
In particular, the modified equivariant Gromov topology and the equivariant Gromov topology coincide in $m_{\Gamma}^{u}(n)$.

Now, every argument of M Bestvina [4] and F Paulin [35; 37] works, and we have the following.

Theorem 2.19 (M Bestvina, F Paulin) The space $\overline{m_{\Gamma}^{u}(n)}$, equipped with the function $m_{\Gamma}^{u}(n) \hookrightarrow \overline{m_{\Gamma}^{u}(n)}$, is a natural compactification of $m_{\Gamma}^{u}(n)$.

By "natural", we mean that the action of $\operatorname{Out}(\Gamma)$ on $m_{\Gamma}^{u}(n)$ extends continuously to an action of $\operatorname{Out}(\Gamma)$ on $\overline{m_{\Gamma}^{u}(n)}$.

We refer to Paulin [37; 36] and Kapovich and Leeb [24] for a complete proof of this result.

2.2.3 Other compactifications We now give a (very) short reminder on the compactification of $X_{\Gamma, \mathrm{SL}(2, \mathbb{R})}$ by J Morgan and P Shalen. The countable collection $\left(f_{\gamma}\right)_{\gamma \in \Gamma}$,

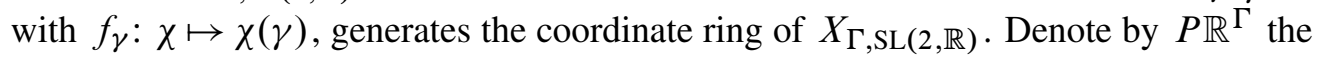
quotient of $[0,+\infty)^{\Gamma} \backslash\{0\}$ by positive multiplication, and let $\theta: X_{\Gamma, \mathrm{SL}(2, \mathbb{R})} \rightarrow P \mathbb{R}^{\Gamma}$ defined by $\theta(x)=\left[\log \left(\left|f_{\gamma}(x)\right|+2\right)\right]_{\gamma \in \Gamma}$. J Morgan and P Shalen proved [32, Proposition I.3.1] that the image of $X_{\Gamma, \mathrm{SL}(2, \mathbb{R})}$ under $\theta$ is relatively compact, so that $\theta$ defines a compactification of $X_{\Gamma, \mathrm{SL}(2, \mathbb{R})}$.

We now restrict to the group $\Gamma=\pi_{1} \Sigma_{g}$ with $g \geq 2$, and we denote by $m_{g}^{u}$ the space $m_{\Gamma}^{u}(2)$. Then the absolute value of the Euler class is defined on $m_{g}^{u}$, and we denote by $m_{g \text {,even }}^{u}$ the subspace of $m_{g}^{u}$ consisting of representations of even Euler class (recall, indeed, that a representation $\rho: \pi_{1} \Sigma_{g} \rightarrow \operatorname{PSL}(2, \mathbb{R})$ lifts to $\operatorname{SL}(2, \mathbb{R})$ if and only if its Euler class is even; we will see that in Section 2.3.4). Then the map $\theta$ factors through $\theta^{\prime}: m_{g \text {,even }}^{u} \rightarrow P \mathbb{R}^{\Gamma}$, which defines a compactification $\overline{m_{g, \text { even }}^{u}}$ MS of $m_{g \text {,even }}^{u}$. Similarly, the functions $m_{g \text {,even }}^{u} \hookrightarrow m_{g}^{u} \hookrightarrow \overline{m_{g}^{u}}$ define a compactification $\overline{m_{g \text {,even }}^{u}}$ of that space. The ideal points of the compactifications $\overline{m_{g \text {,even }}^{u}}$ and $\overline{m_{g \text {,even }}^{u}}$ MS are actions of $\pi_{1} \Sigma_{g}$ on $\mathbb{R}$-trees. These actions on $\mathbb{R}$-trees are irreducible, ie, without global fixed points, or are actions on a line. The topology on $\partial \overline{m_{g}^{u} \text {,even }}$ MS is the axis topology; it is the coarsest topology such that the functions $\ell_{T}(\gamma)=\inf _{x \in T} d(x, \gamma x)$ are continuous. By the main theorem of [36], the spaces $\partial \overline{m_{g \text {,even }}^{u}}$ and $\partial \overline{m_{g, \text { even }}^{u}}$ MS are homeomorphic, and hence, as F Paulin explains it in [35], the spaces $\overline{m_{g \text {,even }}^{u}}$ and $\overline{m_{g}^{u} \text {,even }}$ MS are homeomorphic (we refer to $[32 ; 35 ; 36]$ for details). In particular, Corollary 1.4 concerns simply the space $\overline{m_{g, \text { even }}}$ and it is under that form that we shall prove it in Section 3. 


\subsection{Euler class}

As we mentioned it in the Introduction, the Euler class is a characteristic class which distinguishes the connected components of $\operatorname{Hom}\left(\pi_{1} \Sigma_{g}, \operatorname{PSL}(2, \mathbb{R})\right)$, for $g \geq 2$. It can be defined in the more general context of actions, preserving the order, of any discrete group $\Gamma$ on a set equipped with a total cyclic order, as an element of the cohomology group $H^{2}(\Gamma, \mathbb{Z})$. The definition of the Euler class, in this context, is rather standard. An excellent introduction can be found in Ghys [16] in the context of actions on the circle; and an efficient general overview is given by D Calegari [6, Section 2.3].

In order to prove the continuity of the Euler class (Theorem 1.7), we will need to prove some technical lemmas about it, in Section 2.3.5. For this, it is important to use a computation-oriented definition of this Euler class, which mimics (in a geometric way) the case of actions on a circle. For this reason, we give a self-contained treatment of the Euler class, and for simplicity of the exposition we will soon restrict to the case when $\Gamma$ is the fundamental group of a compact, connected hyperbolic surface, keeping in mind the algorithm given by $\mathrm{J}$ Milnor in [30].

\subsubsection{Cyclically ordered sets}

Definition 2.20 Let $X$ be a set. A (total) cyclic order on $X$ is a function $o: X^{3} \rightarrow$ $\{-1,0,1\}$ such that

(i) $o(x, y, z)=0$ if and only if $\operatorname{Card}\{x, y, z\} \leq 2$;

(ii) for all $x, y$ and $z, o(x, y, z)=o(y, z, x)=-o(x, z, y)$;

(iii) for all $x, y, z$ and $t$, if $o(x, y, z)=1$ and $o(x, z, t)=1$ then $o(x, y, t)=1$.

Remark 2.21 If $o(x, y, z)=1$ and $o(x, z, t)=1$ then we also have $o(x, z, t)=1$ and $o(y, z, t)=1$. Indeed, $o(z, x, y)=o(z, t, x)=1$ so, by condition (iii) of the definition, $o(z, t, y)=1$, that is, $o(y, z, t)=1$. Similarly, $o(x, y, t)=1$ so $o(t, x, y)=1$, which, together with $o(t, y, z)=1$, yields $o(t, x, z)=1$, ie $o(x, z, t)=1$. In other words, the transitivity relation (iii) implies all the other "natural" transitivity relations. In particular, for instance, on a set of 4 elements, there are as many total cyclic orders as injections of that set in the oriented circle, up to orientation-preserving homeomorphism, that is, 6 .

Remark 2.22 A triple $(x, y, z) \in X^{3}$ is called degenerate if $\operatorname{Card}\{x, y, z\} \leq 2$. Thus, a cyclic order $o: X^{3} \rightarrow\{-1,0,1\}$ is determined by its restriction of the set of nondegenerate triples; this restriction is takes values in $\{-1,1\}$ and satisfies conditions (ii) and (iii) of Definition 2.20. This gives an alternative definition of a total cyclic order, which will be used in Section 3. 
In all this text, we use only total cyclic orders (every triple defines an order). Consequently, we will sometimes forget the word "total" when we refer to a cyclic order.

Now fix a set $X$ equipped with a cyclic order $o$ and a base point $x_{0} \in X$. Once this base point $x_{0}$ is fixed, total cyclic orders on $X$ are naturally identified with total orders on $X \backslash\left\{x_{0}\right\}$ :

Definition 2.23 We set $y<_{x_{0}} z$ if $o\left(x_{0}, y, z\right)=1$, and $y \leq_{x_{0}} z$ if $y<_{x_{0}} z$ or $y=z$.

It follows directly from the properties of $o$ that the relation $\leq_{x_{0}}$ is a total order on $X \backslash\left\{x_{0}\right\}$. Reciprocally, if $\leq$ is a total order on $X \backslash\left\{x_{0}\right\}$, then there exists a unique cyclic total order on $X$ which satisfies $o(x, y, z)=1$ for all $x, y, z \neq x_{0}$ such that $x<y<z$, and satisfying $o\left(x_{0}, y, z\right)=1$ as soon as $y<z$. For all $x_{0} \in X$, these two constructions realize a bijection, and its inverse, between the set of total cyclic orders on $X$ and the set of total orders on $X \backslash\left\{x_{0}\right\}$.

When defining the Euler class of an action on the circle, it is essential to consider lifts of homeomorphisms of the circle to homeomorphisms of $\mathbb{R}$. With this in mind, we define the following.

Definition 2.24 On the set $\mathbb{Z} \times X$ we put

- $(m, x)<_{x_{0}}(n, y)$ when $m<n$, for any $x, y \in X$;

- $(k, y)<_{x_{0}}(k, z)$ when $y<_{x_{0}} z$;

- $\left(k, x_{0}\right)<_{x_{0}}(k, y)$ for all $y \in X \backslash\left\{x_{0}\right\}$;

and we put $(m, y) \leq_{x_{0}}(n, z)$ if $(m, y)<_{x_{0}}(n, z)$ or $(m, y)=(n, z)$.

In particular, when restricted to the set $\mathbb{Z} \times\left(X \backslash\left\{x_{0}\right\}\right)$ it is the lexicographic order.

We then check easily that the relation $\leq_{x_{0}}$ is a total order on the set $\mathbb{Z} \times X$.

Example 2.25 If $X=\mathbb{S}^{1}$ and $x_{0} \in \mathbb{S}^{1}$, then $X \backslash\left\{x_{0}\right\}$ is an interval,

$$
\begin{aligned}
\vdots 1\} \times\left(X \backslash\left\{x_{0}\right\}\right) & \vdots\left(2, x_{0}\right) \\
\{0\} \times\left.\left(X \backslash\left\{x_{0}\right\}\right)\right|^{\left(1, x_{0}\right)} & \left(0, x_{0}\right) \\
\vdots-1\} \times\left(X \backslash\left\{x_{0}\right\}\right) & \left(-1, x_{0}\right)
\end{aligned}
$$

and $\mathbb{Z} \times X$ is naturally identified to $\mathbb{R}$; this identification depends canonically on $x_{0}$. 
Another example is the deck of playing cards. It is equipped with a cyclic order, which is preserved as we make a "cut". The choice of the "cut" consists in choosing a card $x_{0}$, and determines a total order in the deck. Here our definition of the order on $\mathbb{Z} \times X$ consists in choosing a cut, and then putting $\mathbb{Z}$ copies of the deck the ones above the others.

2.3.2 Applications and lifts Orientation-preserving homeomorphisms of $\mathbb{S}^{1}$ can be lifted to homeomorphisms of $\mathbb{R}$, in a unique way up to integer translations. Here we will see that the same happens for order-preserving bijections of a cyclically ordered set.

Let $f: X \rightarrow X$, and $\tilde{f}: \mathbb{Z} \times X \rightarrow \mathbb{Z} \times X$ be two functions. If the diagram

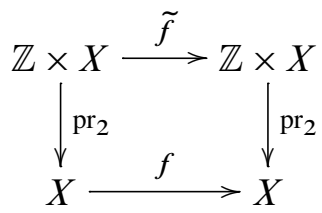

commutes, we say that $\tilde{f}$ is an arbitrary lift of $f$, and that $\tilde{f}$ projects on $f$.

We define an application $h: \mathbb{Z} \times X \rightarrow \mathbb{Z} \times X$ by $h(n, x)=(n+1, x)$.

Proposition 2.26 Let $f: \mathbb{Z} \times X \rightarrow \mathbb{Z} \times X$ be a bijection preserving the order $\leq_{x_{0}}$ and which projects on $\operatorname{Id}_{X}$. Then there exists an integer $n$ such that $f=h^{n}$.

Proof Let $f$ be such a function, and put $n=\operatorname{pr}_{1}\left(f\left(0, x_{0}\right)\right)$. Then the function $\operatorname{pr}_{1} \circ f\left(\cdot, x_{0}\right): \mathbb{Z} \rightarrow \mathbb{Z}$ is a bijection preserving the order, hence for all $k \in \mathbb{Z}$, $f\left(k, x_{0}\right)=\left(n+k, x_{0}\right)$. Now, for all $y \in X \backslash\left\{x_{0}\right\}$, we have $f\left(k, x_{0}\right) \leq_{x_{0}} f(k, y) \leq_{x_{0}}$ $f\left(k+1, x_{0}\right)$, that is, $\left(n+k, x_{0}\right) \leq_{x_{0}} f(k, y) \leq_{x_{0}}\left(n+k+1, x_{0}\right)$, hence $f(k, y)=$ $(n+k, y)$, since $f$ projects on $\operatorname{Id}_{X}$.

Proposition 2.27 For all $x_{0}, x_{1} \in X$, there exists a unique bijection $F_{x_{0} x_{1}}$ of $\mathbb{Z} \times X$ which projects on $\operatorname{Id}_{X}$, such that for all $a, b \in \mathbb{Z} \times X, a<_{x_{0}} b \Leftrightarrow F_{x_{0} x_{1}}(a)<_{x_{1}}$ $F_{x_{0} x_{1}}(b)$, and such that $F_{x_{0} x_{1}}\left(0, x_{0}\right)=\left(0, x_{0}\right)$.

Proof If $x_{0}=x_{1}$, then by the preceding proposition, the unique possible function is $F_{x_{0} x_{1}}=\operatorname{Id}_{\mathbb{Z} \times X}$. Now suppose that $x_{0} \neq x_{1}$. The application $F_{x_{0} x_{1}}$ must project on $\mathrm{Id}_{X}$, so that we can only change the indices, in the way suggested by the following picture. 


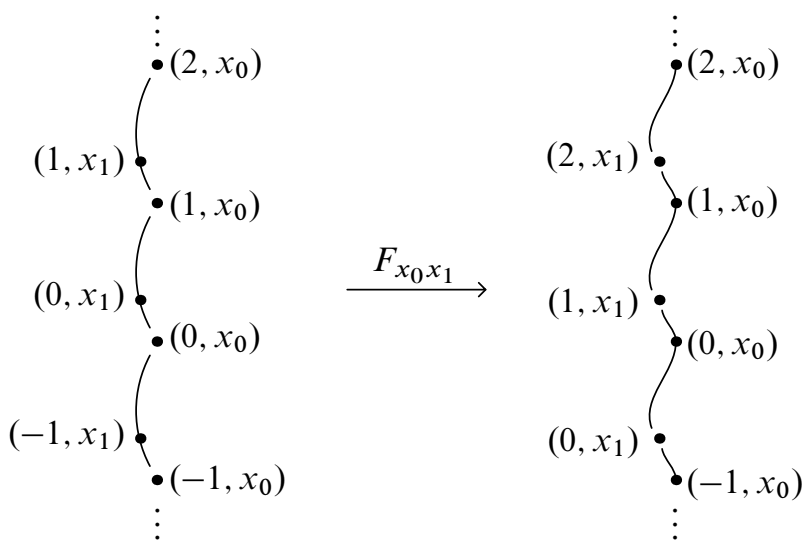

We put $F_{x_{0} x_{1}}\left(k, x_{0}\right)=\left(k, x_{0}\right)$ and $F_{x_{0} x_{1}}\left(k, x_{1}\right)=\left(k+1, x_{1}\right)$. For all $y \in X \backslash\left\{x_{0}, x_{1}\right\}$, if $o\left(x_{0}, x_{1}, y\right)=-1$ we put $F_{x_{0} x_{1}}(k, y)=(k, y)$, otherwise, $F_{x_{0} x_{1}}(k, y)=(k+1, y)$. Then $F_{x_{0} x_{1}}$ satisfies the Proposition; its unicity follows from Proposition 2.26.

Note that for all distinct points $x_{0}, x_{1} \in X$, we have $F_{x_{0} x_{1}} \circ F_{x_{1} x_{0}}=h$, contrary to what our notation may suggest.

Proposition 2.28 Let $X$ be a set equipped with a (total) cyclic order $o$, let $x_{0} \in X$, and let $f: X \rightarrow X$ be a bijection preserving $o$. Then $f$ admits at least one lift $\tilde{f}$ preserving the order $\leq x_{0}$. Moreover, if $\tilde{f}$ and $\tilde{f}^{\prime}$ are two such lifts then there exists $n \in \mathbb{Z}$ such that $\tilde{f}^{\prime}=h^{n} \circ \tilde{f}$.

Proof Let $f: X \rightarrow X$ preserving $o$. We check easily that the map $F: \mathbb{Z} \times X \rightarrow \mathbb{Z} \times X$ defined by $F(n, x)=(n, f(x))$ satisfies: $\forall a, b \in \mathbb{Z} \times X, a \leq_{x_{0}} b \Leftrightarrow F(a) \leq_{f\left(x_{0}\right)} F(b)$. Hence, the map $\tilde{f}=F_{f\left(x_{0}\right) x_{0}} \circ F$ is indeed a lift of $f$, preserving $\leq x_{0}$.

Now, if $\tilde{f}^{\prime}$ is another lift of $f$ preserving $\leq_{x_{0}}$, then $\tilde{f}^{\prime} \circ \tilde{f}^{-1}$ is a lift of $\operatorname{Id}_{X}$ preserving $\leq_{x_{0}}$, hence, by Proposition 2.26, $\tilde{f}^{\prime} \tilde{f}^{-1}=h^{n}$, for some $n \in \mathbb{Z}$.

Now denote by $\operatorname{Ord}(X, o)$ the group of bijections of $X$ which preserve $o$, and by $\widetilde{\operatorname{Ord}}\left(X, o, x_{0}\right)$ the group formed by lifts of elements of $\operatorname{Ord}(X, o)$ to $\mathbb{Z} \times X$ which preserve the order $\leq_{x_{0}}$. By Proposition 2.27, the conjugation by $F_{x_{0} x_{1}}$ realizes a canonical isomorphism between the groups $\widetilde{\operatorname{Ord}}\left(X, o, x_{0}\right)$ and $\widetilde{\operatorname{Ord}}\left(X, o, x_{1}\right)$, for all $x_{0}, x_{1} \in X$. This group, considered up to isomorphism, is denoted by $\widetilde{\operatorname{Ord}}(X, o)$, and if there is no confusion possible about $o$, these two groups are denoted by $\operatorname{Ord}(X)$ and $\widetilde{\operatorname{Ord}}(X)$. 
2.3.3 Euler class Let $(X, o)$ be a cyclically ordered set, let $\Sigma$ be a connected surface, and let $\rho: \pi_{1} \Sigma \rightarrow \operatorname{Ord}(X)$ be a representation: we shall define an element $e(\rho) \in$ $H^{2}(\Sigma, \mathbb{Z})$, which we call the Euler class of the representation.

The choice of an element $x_{0} \in X$ gives rise to the group $\widetilde{\operatorname{Ord}}\left(X, o, x_{0}\right)$, and by Proposition 2.28, the sequence

$$
0 \rightarrow \mathbb{Z} \rightarrow \widetilde{\operatorname{Ord}}\left(X, o, x_{0}\right) \stackrel{p}{\rightarrow} \operatorname{Ord}(X, o) \rightarrow 1
$$

is exact, and defines a central extension of $\operatorname{Ord}(X, o)$ by $\mathbb{Z}$. The canonical map $p$ being onto, we can choose a set theoretical section $s$.

Since we are considering a representation of $\pi_{1} \Sigma$ in a nonabelian group, we need to choose a base point $* \in \Sigma$. Let $C=C_{0} \cup C_{1} \cup C_{2}$, where $C_{i}$ is the set $\left\{\sigma_{\alpha}^{i}\right\}_{\alpha}$ of $i$-cells, be a cellulation of $\Sigma$, where every cell is equipped with an orientation, and suppose that $C_{0}=\{*\}$.

Each loop $\gamma$, based at $*$, in the 1 -skeleton, is equivalent to a word $\left(\sigma_{1}^{1}\right)^{\epsilon_{1}} \cdots\left(\sigma_{k}^{1}\right)^{\epsilon_{k}}$, with $\epsilon_{j}= \pm 1$, in the elements of $C_{1}$. It represents an element of $\pi_{1}(\Sigma, *)$, which we denote by $[\gamma]$. The boundary of any $2-$ cell $\sigma_{\alpha}^{2}$ is a loop $\partial \sigma_{\alpha}^{2}$, based at $*$, as above, well-defined only up to a cyclic permutation. Since $\partial \sigma_{\alpha}^{2}$ is contractible in $\Sigma$, the element of $\widetilde{\operatorname{Ord}}\left(X, o, x_{0}\right)$ :

$$
s\left(\rho\left(\left[\sigma_{1}^{1}\right]\right)\right)^{\epsilon_{1}} \cdots s\left(\rho\left(\left[\sigma_{k}^{1}\right]\right)\right)^{\epsilon_{k}}
$$

is a power of $h$, so it does not depend on the cyclic permutation. By identifying $\mathbb{Z}$ with the group generated by $h$ in $\widetilde{\operatorname{Ord}}\left(X, o, x_{0}\right)$, this defines an integer $n\left(\sigma_{\alpha}^{2}\right)$. Finally, if $c=\sum \lambda_{i} \sigma_{i}^{2}$ is a 2-cycle, we put $e(\rho) \cdot c=\sum \lambda_{i} n\left(\sigma_{i}^{2}\right)$.

Theorem 2.29 For every 2-cycle $c$, the integer $e(\rho) \cdot c$ depends only on $(X, o)$ and on $\rho$; this defines an element $e(\rho) \in H^{2}(\Sigma, \mathbb{Z})$, called the Euler class of $\rho$.

If the surface $\Sigma$ is closed and oriented, then the evaluation of $e(\rho)$ on the fundamental class is an integer, which we still call (abusively) the Euler class of the representation, $e(\rho) \in \mathbb{Z}$.

Proof Step 1 we first prove that given the base points $* \in \Sigma, x_{0} \in X$ and the cellulation, $e(\rho)$ does not depend on the choice of the section $s$. Thus, let $s_{1}$ and $s_{2}$ be two sections of $p$. For every 1-cell $\sigma^{1}$, there exists (by Proposition 2.26) an integer $n\left(\sigma^{1}\right)$ such that $s_{2}\left(\rho\left(\left[\sigma^{1}\right]\right)\right)=s_{1}\left(\rho\left(\left[\sigma^{1}\right]\right)\right) \cdot h^{n\left(\sigma^{1}\right)}$. Since $h$ is central in $\widetilde{\operatorname{Ord}}\left(X, o, x_{0}\right)$, for any 2-cell $\sigma_{\alpha}^{2}$ we have $n_{2}\left(\sigma_{\alpha}^{2}\right)=n_{1}\left(\sigma_{\alpha}^{2}\right)+\sum_{j=1}^{k} \epsilon_{j} n\left(\sigma_{j}^{1}\right)$, with the same notation 
as above, with $n_{1}$ and $n_{2}$ being the integral maps $n$ defined as above by using the sections $s_{1}$ and $s_{2}$, respectively. Therefore, for every 2 -cycle $c$, we have

$$
e(\rho)_{1} \cdot c-e(\rho)_{2} \cdot c=\sum_{\sigma^{2} \in c} \sum_{\sigma^{1} \in \partial \sigma^{2}} n\left(\sigma^{1}\right),
$$

where $e(\rho)_{i}$ is the Euler class defined by the section $s_{i}$. This sum is zero, since $c$ has no boundary.

Step 2 we prove that $e(\rho)$ depends neither on the point $* \in \Sigma$, nor on the cellulation, nor on the base point $x_{0} \in X$. Independence with respect to $*$ follows from the fact that $h$ is central in $\widetilde{\operatorname{Ord}}(X)$, so that no global conjugation of a representation can change its Euler class. Moreover, $e(\rho)$ is defined in terms of the cellulation of $\Sigma$, and hence it is invariant under any isotopy of the cellulation of the surface. Now, let $C=\{*\} \cup C_{1} \cup C_{2}$ be a cellulation of $\Sigma$ with one vertex, and suppose that one of the 2 -cells, say $\sigma^{2}$, is not a triangle. We can therefore define a new cellulation $C^{\prime}$ by adding a 1 -cell $\sigma^{1}$, and replacing the cell $\sigma^{2}$ by the two 2-cells $\sigma_{1}^{2}$ and $\sigma_{2}^{2}$ obtained from cutting $\sigma^{2}$ along $\sigma^{1}$, and with the orientation from $\sigma^{2}$. Then every 2-cycle of $C^{\prime}$ has the same number of $\sigma_{1}^{2}$ and $\sigma_{2}^{2}$ (otherwise it would have a nonzero number of $\sigma^{1}$ in its boundary). Hence, in order to prove that the Euler classes defined by $C$ and $C^{\prime}$ are the same, it suffices to prove that, for some section $s$, we have $n\left(\sigma_{1}^{2}\right)+n\left(\sigma_{2}^{2}\right)=n\left(\sigma^{2}\right)$; but this is immediate from the construction of $n\left(\sigma^{2}\right)$. It is a classical fact that this operation on cellulations (of cutting a nontriangle 2 -cell), together with its inverse and with isotopies, enable to go from any cellulation (with a single 0 -cell) to any other on a same surface (we can even change from any triangulation to any other by using flips); therefore the Euler class does not depend on $C$. Finally, let $x_{0}, x_{0}^{\prime}$ be two points in $X$. The conjugation by $F_{x_{0} x_{0}^{\prime}}$ defines an isomorphism between $\overparen{\operatorname{Ord}}\left(X, o, x_{0}\right)$ and $\widetilde{\operatorname{Ord}}\left(X, o, x_{0}^{\prime}\right)$ descending on the identity on $\operatorname{Ord}(X)$, hence it sends any section $s$ corresponding to $x_{0}$ to a section $s^{\prime}$ corresponding to $x_{0}^{\prime}$, and these sections define the same Euler class because $h$ commutes with $F_{x_{0} x_{0}^{\prime}}$.

Remark 2.30 Let $\Sigma$ be an oriented surface, $\rho$ a representation and $\pi: \Sigma^{\prime} \rightarrow \Sigma$ a covering of degree $d$. Then $e(\rho \circ \pi)=d \cdot e(\rho)$.

Indeed, take a cellulation $C$ of $\Sigma$ and lift it to a cellulation $C^{\prime}$ of $\Sigma^{\prime}$. The cellulation $C^{\prime}$ no longer has a unique 0 -cell, however, any 1 -cell $\sigma^{1}$ of $C^{\prime}$ continues to define an element $\rho\left(\left[\pi\left(\sigma^{1}\right)\right]\right)$, and we can define a number $e(\rho \circ \pi)$ in that way here. Now, we can prove that this number is invariant under removal of some 1 -cells, and under the merging of several 1 -cells attached by a 0 -cell, in a similar fashion as we did in Step 2 of the proof of Theorem 2.29; we leave the details as an exercise to the reader. 
The choice of defining the Euler class (for actions on cyclically ordered sets) in terms of the cohomology of the surface is motivated by Milnor's algorithm and it gives a straightforward proof of it (see the next Section). However, the same construction can be given in terms of the cohomology of the group $\pi_{1} \Sigma$ : all the sections of the projection $\widetilde{\operatorname{Ord}}\left(X, o, x_{0}\right) \rightarrow \operatorname{Ord}(X)$ define the same element of $H^{2}(\operatorname{Ord}(X), \mathbb{Z})$, which, pulled back to $H^{2}\left(\pi_{1} \Sigma, \mathbb{Z}\right)$, defines, under the identification $H^{2}\left(\pi_{1} \Sigma, \mathbb{Z}\right) \simeq H^{2}(\Sigma, \mathbb{Z})$, the Euler class we have just constructed. We refer to Ghys [16, Sections 6.1 and 6.2] for more details. In particular, we have the following:

Remark 2.31 Let $\Gamma$ be a group such that $H^{2}(\Gamma, \mathbb{Z})=0$, and let $\rho_{1}: \pi_{1} \Sigma_{g} \rightarrow \Gamma$, $\rho_{2}: \Gamma \rightarrow \operatorname{Ord}(X, o)$. Then $e\left(\rho_{1} \circ \rho_{2}\right)=0$.

2.3.4 Milnor's algorithm Consider a closed, oriented surface of genus $g, \Sigma_{g}$, and equip it with a "standard" cellulation, featuring a single 2 -cell, a single vertex and $4 g$ edges labelled by $a_{i}, b_{i}, a_{i}^{-1}$ and $b_{i}^{-1}$, for $1 \leq i \leq g$. It yields a standard presentation $\pi_{1} \Sigma_{g}=\left\langle a_{1}, \ldots, b_{g} \mid \Pi_{i}\left[a_{i}, b_{i}\right]=1\right\rangle$. Then a representant of the fundamental class $c \in H_{2}\left(\pi_{1} \Sigma_{g}, \mathbb{Z}\right)$ is the 2 -cycle consisting of the unique 2-cell, equipped with its orientation.

Given a representation $\rho \in \operatorname{Hom}\left(\pi_{1} \Sigma_{g}, \operatorname{Ord}(X, o)\right)$, take some $x_{0} \in X$ and choose an arbitrary lift $\widetilde{\rho(x)}$ for all $x \in\left\{a_{1}, b_{1}, \ldots, a_{g}, b_{g}\right\}$. As we said before, we still denote by $e(\rho) \in \mathbb{Z}$ the evaluation of $e(\rho) \in H^{2}\left(\Sigma_{g}, \mathbb{Z}\right)$ on the fundamental class $c$. Thus, by construction of the Euler class we have

$$
\left[\widetilde{\rho\left(a_{1}\right)}, \widetilde{\rho\left(b_{1}\right)}\right] \cdots\left[\widetilde{\rho\left(a_{g}\right)}, \widetilde{\rho\left(b_{g}\right)}\right]=h^{e(\rho)} \text {. }
$$

Since $h$ is central in $\widetilde{\operatorname{Ord}}\left(X, o, x_{0}\right)$ and commutes with $F_{x_{0} x_{1}}$ for all $x_{1} \in X \backslash\left\{x_{0}\right\}$, the result of this product of commutators does not depend on $x_{0}$ neither on the choices of the lifts $\widetilde{\rho\left(a_{i}\right)}, \widetilde{\rho\left(b_{i}\right)}$, which could be made according to any section $s: \operatorname{Ord}(X) \rightarrow$ $\widetilde{\operatorname{Ord}}\left(X, o, x_{0}\right)$.

Let us come back to Example 2.25. The group $\operatorname{Ord}\left(\mathbb{S}^{1}\right)$ is just $\mathrm{Homeo}^{+}\left(\mathbb{S}^{1}\right)$, and its lift $\widetilde{\operatorname{Ord}}(X)$ is identified with the group $\operatorname{Homeo}_{1}^{+}(\mathbb{R})$ of increasing bijections of $\mathbb{R}$ which commute with integer translations. The group $\operatorname{PSL}(2, \mathbb{R})$ of orientationpreserving isometries of $\mathbb{H}^{2}$ acts faithfully on the circle $\partial \mathbb{H}^{2}$; this defines an inclusion $\operatorname{PSL}(2, \mathbb{R}) \subset \operatorname{Ord}\left(\mathbb{S}^{1}\right)$, and the subgroup of $\operatorname{Homeo}_{1}^{+}(\mathbb{R})$ of lifts of $\operatorname{PSL}(2, \mathbb{R})$ turns out to be its universal cover, $\widehat{\operatorname{PSL}(2, \mathbb{R})}$. In this context, the central extension used in Section 2.3.3 is also the extension of $\operatorname{PSL}(2, \mathbb{R})$ by its fundamental group, isomorphic to $\mathbb{Z}$. Therefore, in the formula (2-1), $h$ is identified with a generator of $\pi_{1}(\operatorname{PSL}(2, \mathbb{R}))$. In the intermediate cover $\operatorname{SL}(2, \mathbb{R})$ of $\operatorname{PSL}(2, \mathbb{R})$, the image of $h$ is $-\mathrm{Id}$. It follows that a representation $\rho \in \operatorname{Hom}\left(\pi_{1} \Sigma_{g}, \operatorname{PSL}(2, \mathbb{R})\right)$ lifts to $\operatorname{SL}(2, \mathbb{R})$ if and only if $e(\rho)$ is even. 
2.3.5 Finite sets suffice Denote by $\mathbb{F}_{2 g}$ the free group on the set $\left\{a_{1}, b_{1}, \ldots, a_{g}, b_{g}\right\}$, and let $w=\left[a_{1}, b_{1}\right] \cdots\left[a_{g}, b_{g}\right]$. The images under the canonical surjection $\mathbb{F}_{2 g} \rightarrow$ $\pi_{1} \Sigma_{g}$ of the subwords of $w$ form a set $P$, and in all the sequel of this text we denote by $P_{\text {ref }}$ the set $P \cup P^{-1}$. A major interest of Milnor's algorithm is that we need only finitely many pieces of information, concerning the action of the finite set $P_{\text {ref }}$ on the ordered set $X$, in order to be able to compute the Euler class of a representation. This is the key idea which will prove, in Section 3, that the Euler class extends continuously to the boundary of $m_{g}^{o}$.

More precisely, the idea is the following:

Proposition 2.32 Let $(X, o)$ and $\left(X^{\prime}, o^{\prime}\right)$ be two cyclically ordered sets, equipped with base points $x_{0}, x_{0}^{\prime}$. Let $\rho: \pi_{1} \Sigma_{g} \rightarrow \operatorname{Ord}(X, o)$ and $\rho^{\prime}: \pi_{1} \Sigma_{g} \rightarrow \operatorname{Ord}\left(X^{\prime}, o^{\prime}\right)$ be two representations. Suppose that for all $g_{1}, g_{2}, g_{3} \in P_{\text {ref }}$,

$$
o\left(g_{1} x_{0}, g_{2} x_{0}, g_{3} x_{0}\right)=o^{\prime}\left(g_{1} x_{0}, g_{2} x_{0}, g_{3} x_{0}\right) .
$$

Then $e\left(\rho_{1}\right)=e\left(\rho_{2}\right)$.

In fact we shall need a slightly more subtle statement, since we want the Euler class to be stable under small degenerations. We have stated Proposition 2.32 in order to fix the ideas, but we will not use it, and we leave its proof as an (easy) exercise. Instead we will prove the following:

Proposition 2.33 Let $(X, o)$ and $\left(X^{\prime}, o^{\prime}\right)$ be two cyclically ordered sets, equipped with base points $x_{0}, x_{0}^{\prime}$. Let $\rho: \pi_{1} \Sigma_{g} \rightarrow \operatorname{Ord}(X, o)$ and $\rho^{\prime}: \pi_{1} \Sigma_{g} \rightarrow \operatorname{Ord}\left(X^{\prime}, o^{\prime}\right)$ be two representations. Let $y_{0} \in X$ and $y_{0}^{\prime} \in X^{\prime}$. Suppose also that $x_{0} \notin P_{\text {ref }} \cdot y_{0}$, that $\operatorname{Card}\left(P_{\text {ref }} \cdot y_{0}\right) \geq 2$, and that for all $g_{1}, g_{2}, g_{3} \in P_{\text {ref }}$,

and

$$
o\left(g_{1} x_{0}, g_{2} x_{0}, g_{3} y_{0}\right)=1 \Rightarrow o^{\prime}\left(g_{1} x_{0}^{\prime}, g_{2} x_{0}^{\prime}, g_{3} y_{0}^{\prime}\right)=1
$$

Then $e\left(\rho_{1}\right)=e\left(\rho_{2}\right)$.

Everything relies on the two following elementary lemmas:

Lemma 2.34 Let $(X, o)$ be a cyclically ordered set, and $f \in \operatorname{Ord}(X)$. Suppose we have a base point $x_{0} \in X$ and an element $y \in X \backslash\left\{x_{0}\right\}$ such that $f(y) \neq x_{0}$. Denote by $\tilde{f}$ the lift of $f$ to $\mathbb{Z} \times X$ satisfying $\tilde{f}\left(0, x_{0}\right)=\left(0, f\left(x_{0}\right)\right)$, and denote by $n$ the integer such that $\tilde{f}(0, y)=(n, f(y))$. Then $n$ depends only on $o\left(x_{0}, f\left(x_{0}\right), f(y)\right)$. More precisely, $n=\max \left(0,-o\left(x_{0}, f\left(x_{0}\right), f(y)\right)\right)$. 
Lemma 2.35 Let $(X, o)$ be a cyclically ordered set, $f \in \operatorname{Ord}(X)$ and $x_{0} \in X$. Let $x_{1}, x_{2} \in X$ be such that $o\left(x_{0}, x_{1}, x_{2}\right)=o\left(f\left(x_{0}\right), x_{1}, x_{2}\right)=1$. Then there exists a lift $\widetilde{f}$ of $f$ to $\mathbb{Z} \times X$ such that $\left(-1, x_{2}\right)<x_{0} \tilde{f}\left(0, x_{0}\right)<x_{0}\left(0, x_{1}\right)$. Moreover, if $y \in X$ is such that $o\left(x_{1}, x_{2}, y\right) \leq 0$ and $o\left(x_{1}, x_{2}, f(y)\right) \leq 0$, then this lift $\tilde{f}$ satisfies $\widetilde{f}(0, y)=(0, f(y))$.

Proof of Lemma 2.34 We have $\underset{\sim}{f}(y) \neq f\left(x_{0}\right)$ hence $y \neq x_{0}$ and hence $\left(0, x_{0}\right)<x_{0}$ $(0, y)<_{x_{0}}\left(1, x_{0}\right)$. The function $\tilde{f}$ is increasing, so that $\left(0, f\left(x_{0}\right)\right)<_{x_{0}}(n, f(y))<_{x_{0}}$ $\left(1, f\left(x_{0}\right)\right)$.

If $o\left(x_{0}, f\left(x_{0}\right), f(y)\right)=0$ then $x_{0}=f\left(x_{0}\right)$ and hence $n=0$.

If $o\left(x_{0}, f\left(x_{0}\right), f(y)\right)=1$ then $\left(0, f\left(x_{0}\right)\right)<_{x_{0}}(0, f(y))<_{x_{0}}\left(1, f\left(x_{0}\right)\right)$ and then $n=0$.

If $o\left(x_{0}, f\left(x_{0}\right), f(y)\right)=-1$ then $\left(-1, f\left(x_{0}\right)\right)<_{x_{0}}(0, f(y))<_{x_{0}}\left(0, f\left(x_{0}\right)\right)$ and in that case $n=1$.

Proof of Lemma 2.35 There are three cases to consider here.

- If $f\left(x_{0}\right)=x_{0}$, then $\left(-1, x_{2}\right)<x_{0}\left(0, f\left(x_{0}\right)\right)<x_{0}\left(0, x_{1}\right)$, so we take $\tilde{f}$ such that $\tilde{f}\left(0, x_{0}\right)=\left(0, f\left(x_{0}\right)\right)$. We then have $\left(0, x_{0}\right)<_{x_{0}}(0, y)<_{x_{0}}\left(1, x_{0}\right)$, hence, by applying $\tilde{f}$ (which is strictly increasing): $\left(0, x_{0}\right)<_{x_{0}} \tilde{f}(0, y)<_{x_{0}}\left(1, x_{0}\right)$, so that $\widetilde{f}(0, y)=(0, f(y))$.

- If $o\left(x_{0}, x_{1}, f\left(x_{0}\right)\right)=-1$ then $\left(-1, x_{2}\right)<_{x_{0}}\left(0, x_{0}\right)<_{x_{0}}\left(0, f\left(x_{0}\right)\right)<_{x_{0}}\left(0, x_{1}\right)$ so we take again $\tilde{f}$ such that $\tilde{f}\left(0, x_{0}\right)=\left(0, f\left(x_{0}\right)\right)$. By Lemma 2.34, it suffices to prove that $o\left(x_{0}, f\left(x_{0}\right), f(y)\right) \geq 0$ in order to have $n=0$ and thus $\tilde{f}(0, y)=(0, f(y))$. But if $o\left(x_{0}, f(y), f\left(x_{0}\right)\right)=1$, since $o\left(x_{0}, f\left(x_{0}\right), x_{1}\right)=1$ we get $o\left(x_{0}, f(y), x_{1}\right)=1$ so $o\left(x_{1}, x_{0}, f(y)\right)=1$ which, together with $o\left(x_{1}, x_{2}, x_{0}\right)=1$, gives $o\left(x_{1}, x_{2}, f(y)\right)=1$, a contradiction.

- If $o\left(x_{0}, x_{1}, f\left(x_{0}\right)\right)=1$ then we have $o\left(x_{0}, x_{2}, f\left(x_{0}\right)\right)=1$ (indeed, this follows from the equalities $\left.o\left(f\left(x_{0}\right), x_{0}, x_{1}\right)=o\left(f\left(x_{0}\right), x_{1}, x_{2}\right)=1\right)$, hence

$$
\left(-1, x_{2}\right)<_{x_{0}}\left(-1, f\left(x_{0}\right)\right)<_{x_{0}}\left(0, x_{0}\right)<_{x_{0}}\left(0, x_{1}\right)
$$

and we take $\tilde{f}$ such that $\tilde{f}\left(0, x_{0}\right)=\left(-1, f\left(x_{0}\right)\right)$. In particular, $\tilde{f}=\tilde{f}^{\prime} \circ h^{-1}$, where $\tilde{f}^{\prime}\left(0, x_{0}\right)=\left(0, f\left(x_{0}\right)\right)$. And we have $o\left(x_{0}, f\left(x_{0}\right), f(y)\right)=-1$ (indeed, if $f(y)=x_{1}$ or $x_{2}$ we already have this equality, and otherwise $o\left(x_{2}, x_{1}, f(y)\right)=1$, which, together with the equality $o\left(x_{2}, x_{0}, x_{1}\right)=1$, gives $o\left(x_{2}, x_{0}, f(y)\right)=1$, ie $o\left(x_{0}, f(y), x_{2}\right)=1$, which, together with $o\left(x_{0}, x_{2}, f\left(x_{0}\right)\right)=1$, yields $\left.o\left(x_{0}, f(y), f\left(x_{0}\right)\right)=1\right)$ therefore Lemma 2.34 applied to $\widetilde{f}^{\prime}$ implies that $\tilde{f}^{\prime}(0, y)=(1, f(y))$, whence $\tilde{f}(0, y)=$ $(0, f(y))$ once again. 
Proof of Proposition 2.33 Denote $y_{i}=\left[\rho\left(a_{i+1}\right), \rho\left(b_{i+1}\right)\right] \cdots\left[\rho\left(a_{g}\right), \rho\left(b_{g}\right)\right] \cdot y_{0}$. Then in particular $y_{g}=y_{0}$. The integers $m_{i}$ such that

$$
\left.\widetilde{\rho\left(a_{i}\right)}, \widetilde{\rho\left(b_{i}\right)}\right]\left(0, y_{i}\right)=\left(m_{i}, y_{i-1}\right)
$$

do not depend on the choices of the lifts $\widetilde{\rho\left(a_{i}\right)}, \widetilde{\rho\left(b_{i}\right)}$, and $e(\rho)=\sum_{i=1}^{g} m_{i}$, by Milnor's algorithm. We also use similar notation in $X^{\prime}$.

The finite set $P_{\text {ref }} \cdot y_{0} \subset X \backslash\left\{x_{0}\right\}$, equipped with the order $<_{x_{0}}$, contains a smallest element $x_{1}$ and a biggest element $x_{2}$. Similarly we define $x_{1}^{\prime}$ and $x_{2}^{\prime}$ in $X^{\prime}$. Let then $\gamma_{1}$ be an element of $P_{\text {ref }}$ such that $\gamma_{1} y_{0}^{\prime}=x_{1}^{\prime}$. Then for all $\gamma \in P_{\text {ref }}, o^{\prime}\left(x_{0}^{\prime}, \gamma_{1} y_{0}^{\prime}, \gamma y_{0}^{\prime}\right) \leq 0$, hence $o\left(x_{0}, \gamma_{1} y_{0}, \gamma y_{0}\right) \leq 0$, so that $\gamma_{1} y_{0}$ is minimal among $P_{\text {ref }} \cdot y_{0}$ in $X \backslash\left\{x_{0}\right\}$ for the order $<_{x_{0}}$, that is, $\gamma_{1} y_{0}=x_{1}$. Similarly, $x_{2}$ and $x_{2}^{\prime}$ correspond to (at least) one same element $\gamma_{2} \in P_{\text {ref }}$. Moreover, $\operatorname{since} \operatorname{Card}\left(P_{\text {ref }} \cdot y_{0}\right) \geq 2$, we have $x_{1}<_{x_{0}} x_{2}$, hence $o\left(x_{0}, x_{1}, x_{2}\right)=1$.

For every element $\gamma \in\left\{a_{1}, b_{1}, \ldots, a_{g}, b_{g}\right\}$ we define $\widetilde{\rho(\gamma)}$ and $\widetilde{\rho^{\prime}(\gamma)}$ as follows. If $\rho(\gamma) \cdot x_{0} \neq x_{0}$, we choose $\widetilde{\rho(\gamma)}$ such that $\widetilde{\rho(\gamma)}\left(0, x_{0}\right)=\left(0, \rho(\gamma) \cdot x_{0}\right)$; and we choose $\widehat{\rho^{\prime}(\gamma)}$ such that $\widehat{\rho^{\prime}(\gamma)}\left(0, x_{0}^{\prime}\right)=\left(0, \rho^{\prime}(\gamma) \cdot x_{0}^{\prime}\right)$. Otherwise, if $\rho(\gamma) \cdot x_{0}=x_{0}$ then we have $o\left(x_{0}, x_{1}, x_{2}\right)=o\left(\rho(\gamma) \cdot x_{0}, x_{1}, x_{2}\right)=1$ hence, by Lemma 2.35, $\rho(\gamma)$ possesses a lift $\widetilde{\rho(\gamma)}$ such that $\left(-1, x_{2}\right)<_{x_{0}} \widetilde{\rho(\gamma)}\left(0, x_{0}\right)<_{x_{0}}\left(0, x_{1}\right)$, and in that case again we have $o^{\prime}\left(x_{0}^{\prime}, x_{1}^{\prime}, x_{2}^{\prime}\right)=o^{\prime}\left(\rho^{\prime}(\gamma) \cdot x_{0}^{\prime}, x_{1}^{\prime}, x_{2}^{\prime}\right)=1$ (indeed, $o\left(x_{0}, x_{1}, x_{2}\right)=$ $o\left(x_{0}, \gamma_{1} y_{0}, \gamma_{2} y_{0}\right)=1$ so that $o^{\prime}\left(x_{0}^{\prime}, x_{1}^{\prime}, x_{2}^{\prime}\right)=1$ and, similarly, $o\left(\gamma x_{0}, x_{1}, x_{2}\right)=1$ so $o^{\prime}\left(\rho^{\prime}(\gamma) x_{0}^{\prime}, x_{1}^{\prime}, x_{2}^{\prime}\right)=1$, since $\left.\gamma, \gamma_{1}, \gamma_{2} \in P_{\text {ref }}\right)$. Therefore, still by applying Lemma 2.35, we can define $\widetilde{\rho^{\prime}(\gamma)}$ in such a way that $\left(-1, x_{2}^{\prime}\right)<_{x_{0}^{\prime}} \widehat{\rho^{\prime}(\gamma)}\left(0, x_{0}^{\prime}\right)<_{x_{0}^{\prime}}\left(0, x_{1}^{\prime}\right)$.

Now denote by $n_{i_{1}}, n_{i_{2}}, n_{i_{3}}, n_{i_{4}}$ the integers such that

$$
\begin{aligned}
\left.\widetilde{\rho\left(b_{i}\right)}\right)^{-1}\left(0, y_{i}\right) & =\left(n_{i_{4}}, \rho\left(b_{i}\right)^{-1} \cdot y_{i}\right), \\
\left.\widetilde{\rho\left(a_{i}\right)}\right)^{-1} \cdot\left(0, \rho\left(b_{i}\right)^{-1} \cdot y_{i}\right) & =\left(n_{i_{3}}, \rho\left(a_{i}\right)^{-1} \rho\left(b_{i}\right)^{-1} \cdot y_{i}\right),
\end{aligned}
$$

and similarly we define integers $n_{i_{1}}^{\prime}, \ldots, n_{i_{4}}^{\prime}$.

Let us first check that $n_{i_{4}}=n_{i_{4}}^{\prime}$. Denote $\gamma=\left[a_{i+1}, b_{i+1}\right] \cdots\left[a_{g}, b_{g}\right]$ (that is the element of $\pi_{1} \Sigma_{g}$ defined by the subword following $b_{i-1}$ in $w$ ).

- If $\rho\left(b_{i}\right) \cdot x_{0} \neq x_{0}$, then $o\left(x_{0}, \rho\left(b_{i}^{-1}\right) \cdot x_{0}, \rho\left(b_{i}^{-1} \gamma\right) \cdot y_{0}\right) \neq 0$ (indeed, these three points are all distinct since $\gamma$ and $b_{i}^{-1} \gamma$ are in $P_{\text {ref }}$ ), and hence

$$
o^{\prime}\left(x_{0}^{\prime}, \rho^{\prime}\left(b_{i}^{-1}\right) \cdot x_{0}^{\prime}, \rho^{\prime}\left(b_{i}^{-1}\right) \cdot y_{i}^{\prime}\right)=o\left(x_{0}, \rho\left(b_{i}^{-1}\right) \cdot x_{0}, \rho\left(b_{i}^{-1}\right) \cdot y_{i}\right)
$$

so by Lemma 2.34 (applied to $f=\rho\left(b_{i}^{-1}\right), y=y_{i}$ and to $f=\rho^{\prime}\left(b_{i}^{-1}\right)$ and $y=y_{i}^{\prime}$ ) we have $n_{i_{4}}=n_{i_{4}}^{\prime}$. 
- If $\rho\left(b_{i}\right) \cdot x_{0}=x_{0}$, then by Lemma 2.35, this time we have $n_{i_{4}}=n_{i_{4}}^{\prime}=0$.

Similarly we get $n_{i_{3}}-n_{i_{4}}=n_{i_{3}}^{\prime}-n_{i_{4}}^{\prime}, \ldots, n_{i_{1}}-n_{i_{2}}=n_{i_{1}}^{\prime}-n_{i_{2}}^{\prime}$, so that $m_{i}=m_{i}^{\prime}$, and hence $e(\rho)=e\left(\rho^{\prime}\right)$.

\subsection{Almost faithful morphisms}

The connectedness of $\overline{m_{g}^{u}}$ (Theorem 3.35) strongly relies on a property of surface groups related to the fact that these groups are "limit groups" (see eg Sela [39], Guirardel [22] and Champetier and Guirardel [7]).

Let us fix some notation first. Fix a standard presentation of the fundamental group

$$
\pi_{1} \Sigma_{g}=\left\langle a_{1}, \ldots, b_{g} \mid\left[a_{1}, b_{1}\right] \cdots\left[a_{g}, b_{g}\right]=1\right\rangle
$$

of the surface $\Sigma_{g}$. The set $S=\left\{a_{1}, \ldots, b_{g}\right\}$ generates $\pi_{1} \Sigma_{g}$, and we denote by $B_{n} \subset \pi_{1} \Sigma_{g}$ the ball of centre 1 and radius $n$ for the Cayley metric associated to the generating set $S$. Also, we denote by $\mathbb{F}_{k}$ a free group of rank $k$.

A group $\Gamma$ is said to be residually free if for all $\gamma \in \Gamma \backslash\{1\}$, there exists a morphism $\varphi: \Gamma \rightarrow \mathbb{F}_{2}$ such that $\varphi(\gamma) \neq 1$. We say that $\Gamma$ is fully residually free if for every finite subset $\left\{\gamma_{1}, \ldots, \gamma_{n}\right\} \subset \Gamma \backslash\{1\}$, there exists a morphism $\varphi: \Gamma \rightarrow \mathbb{F}_{2}$ such that for all $i \in\{1, \ldots, n\}, \varphi\left(\gamma_{i}\right) \neq 1$. We will use the following celebrated result:

Theorem 2.36 (G Baumslag [2]) For all $g \geq 2$, the group $\pi_{1} \Sigma_{g}$ is fully residually free. In other words, for every $n \geq 0$, there exists a morphism $\varphi_{n}: \pi_{1} \Sigma_{g} \rightarrow \mathbb{F}_{2}$ such that $\operatorname{ker}\left(\varphi_{n}\right) \cap B_{n}=\{1\}$.

Heuristically, the morphisms $\varphi_{n}$ are "more and more injective". In the language of [39; 7], the group $\pi_{1} \Sigma_{g}$ is a "limit group" of the group $\mathbb{F}_{2}$. In fact, we will also need a statement a little more precise: we will need to make explicitly given morphisms "more and more injective", by composing them with automorphisms of $\pi_{1} \Sigma_{g}$. Let us describe these morphisms here.

For all $g \geq 3$, we denote by $e_{g}: \pi_{1} \Sigma_{g} \rightarrow \pi_{1} \Sigma_{g-1}$ the morphism consisting of collapsing the last handle. More precisely, given the two standard presentations

$$
\begin{aligned}
\pi_{1} \Sigma_{g} & =\left\langle a_{1}, \ldots, b_{g} \mid \prod_{i=1}^{g}\left[a_{i}, b_{i}\right]=1\right\rangle, \\
\pi_{1} \Sigma_{g-1} & =\left\langle a_{1}, \ldots, b_{g-1} \mid \prod_{i=1}^{g-1}\left[a_{i}, b_{i}\right]=1\right\rangle,
\end{aligned}
$$


the map $e_{g}$ is defined by $e_{g}(\gamma)=\gamma$ for $\gamma=a_{1}, b_{1}, \ldots, a_{g-1}, b_{g-1}$ and $e_{g}(\gamma)=1$ for $\gamma=a_{g}, b_{g}$.

Now let $g \geq 2$; we will use a cocompact Fuchsian group $G_{g} \subset \operatorname{PSL}(2, \mathbb{R})$ and a morphism $p_{g}: \pi_{1} \Sigma_{g} \rightarrow G_{g}$, both depending on the parity of $g$.

If $g$ is even, $g=2 g^{\prime}$, consider a cocompact Fuchsian group $G_{g}$ of signature $\left(g^{\prime} ; 2\right)$ (we use the notation of [25] here). Recall that this is the fundamental group of the hyperbolic orbifold of genus $g^{\prime}$ with one conic singularity of angle $\pi(=2 \pi / 2)$. It has the presentation

$$
G_{g}=\left\langle\alpha_{1}, \ldots, \beta_{g^{\prime}} \mid\left(\left[\alpha_{1}, \beta_{1}\right] \cdots\left[\alpha_{g^{\prime}}, \beta_{g^{\prime}}\right]\right)^{2}=1\right\rangle .
$$

Then we define the map $p_{g}: \pi_{1} \Sigma_{g} \rightarrow G_{g}$ by letting $p_{g}\left(a_{i}\right)=p_{g}\left(a_{g^{\prime}+i}\right)=\alpha_{i}$ and $p_{g}\left(b_{i}\right)=p_{g}\left(b_{g^{\prime}+i}\right)=\beta_{i}$ for all $i$ between 1 and $g^{\prime}$.

In the case when $g$ is odd, $g=2 g^{\prime}+1$, we fix a cocompact Fuchsian group $G_{g}$ of signature $\left(g^{\prime} ; 2,2,2\right)$. It has the following presentation:

$$
G_{g}=\left\langle q_{1}, q_{2}, \alpha_{1}, \ldots, \beta_{g^{\prime}} \mid q_{1}^{2}=q_{2}^{2}=1,\left(q_{1} q_{2}\left[\alpha_{1}, \beta_{1}\right] \cdots\left[\alpha_{g^{\prime}}, \beta_{g^{\prime}}\right]\right)^{2}=1\right\rangle .
$$

We denote by $p_{g}: \pi_{1} \Sigma_{g} \rightarrow G_{g}$ the morphism defined by $p_{g}\left(a_{1}\right)=q_{1}^{-1}, p_{g}\left(b_{1}\right)=q_{2}^{-1}$, and $p_{g}\left(a_{i}\right)=\left(q_{1} q_{2}\right)^{-1} \alpha_{i-1}\left(q_{1} q_{2}\right), p_{g}\left(b_{i}\right)=\left(q_{1} q_{2}\right)^{-1} \beta_{i-1}\left(q_{1} q_{2}\right)$, for all $i$ between 2 and $g^{\prime}+1$, and $p_{g}\left(a_{i}\right)=\alpha_{i-g^{\prime}-1}$ and $p_{g}\left(b_{i-g^{\prime}-1}\right)=\beta_{i}$ when $g^{\prime}+2 \leq i \leq g$.

In the two cases of parity of $g$, the discrete representation $p_{g}$ (as an element of $R_{g}$ ) was proved [13, Proposition 4.5] to have Euler class $2 g-3$; this is why we consider it.

Lemma 2.37 Let $g \geq 4$. Then for all $n \geq 0$, there exists an element $\gamma_{n} \in \operatorname{Aut}\left(\pi_{1} \Sigma_{g}\right)$ such that the kernel of the morphism $p_{g} \circ \gamma_{n}: \pi_{1} \Sigma_{g} \rightarrow G_{g}$ does not contain any nontrivial element of length less than $n$.

Similarly:

Lemma 2.38 Let $g \geq 3$. Then for all $n \geq 0$, there exists an element $\gamma_{n} \in \operatorname{Aut}\left(\pi_{1} \Sigma_{g}\right)$ such that the kernel of the map $e_{g} \circ \gamma_{n}: \pi_{1} \Sigma_{g} \rightarrow \pi_{1} \Sigma_{g-1}$ does not contain any nontrivial elements of length less than $n$.

V Guirardel pointed out to me the following proof, which is due to Z Sela.

Proposition 2.39 Let $\Sigma$ be a compact, connected, orientable surface, possibly with boundary, of Euler characteristic less or equal to -1 . Let $\varphi: \pi_{1} \Sigma \rightarrow \mathbb{F}$ be a morphism of nonabelian image in a free group $\mathbb{F}$, whose restrictions to the fundamental groups of 
the boundary components are injective. Then for all finite subset $P \subset \pi_{1} \Sigma \backslash\{1\}$, there exists a diffeomorphism $\gamma_{P}$ of $\Sigma$, preserving pointwise the boundary components, and such that $\operatorname{ker}\left(\varphi \circ \gamma_{P *}\right) \cap P=\varnothing$.

Corollary 2.40 Let $g \geq 2$, and let $\varphi: \pi_{1} \Sigma_{g} \rightarrow \mathbb{F}$ be a morphism of nonabelian image. Then for all $n$, there exists $\gamma_{n} \in \operatorname{Aut}\left(\pi_{1} \Sigma_{g}\right)$ such that $\operatorname{ker}\left(\varphi \circ \gamma_{n}\right) \cap B_{n}=\{1\}$.

Lemma 2.37 and Lemma 2.38 follow:

Proof of Lemma 2.37 The map $\varphi_{g}: G_{g} \rightarrow \mathbb{F}_{2}$ defined by $\varphi_{g}\left(\alpha_{1}\right)=x, \varphi_{g}\left(\alpha_{2}\right)=y$ and $\varphi_{g}(u)=1$ for all the other generators $u$ of $G_{g}$, with $\mathbb{F}_{2}=\langle x, y\rangle$, is a morphism of nonabelian image, and $\varphi_{g} \circ p_{g}: \pi_{1} \Sigma_{g} \rightarrow \mathbb{F}_{2}$ is therefore a morphism satisfying the hypotheses of Corollary 2.40. Thus, we can conjugate it by automorphisms of $\pi_{1} \Sigma_{g}$ in order to make it "arbitrarily injective".

Proof of Lemma 2.38 If $g \geq 3$, the map $\varphi_{g}: \pi_{1} \Sigma_{g-1} \rightarrow \mathbb{F}_{2}$ defined by $\varphi_{g}\left(a_{1}\right)=x$, $\varphi_{g}\left(a_{2}\right)=y$ and $\varphi_{g}(u)=1$ for all the other generators $\pi_{1} \Sigma_{g-1}$ is still a morphism of nonabelian image.

The proof of the proposition relies on the following two lemmas.

Lemma 2.41 (Z Sela [39, Lemma 5.13]) Let $\Sigma$ be a compact, connected, orientable surface, possibly with boundary, of Euler characteristic less or equal to -1 . Let $\varphi: \pi_{1} \Sigma \rightarrow \mathbb{F}$ be a morphism of nonabelian image in a free group $\mathbb{F}$, whose restrictions to the fundamental groups of the boundary components are injective. Then there exists a family of disjoint closed simple curves $c_{1}, \ldots, c_{p}$ in $\Sigma$, which cut $\Sigma$ into pairs of pants, and such that the restriction of $\varphi$ to the fundamental group of each pair of pant is injective.

Lemma 2.42 (G Baumslag [2, Proposition 1]) Let $\mathbb{F}$ be a free group and let $a_{1}, \ldots, a_{n}$, $c \in \mathbb{F}$ be such that $c$ does not commute with any of the $a_{i}$ 's. Then for all $k_{0}, \ldots, k_{n}$ large enough, the element $c^{k_{0}} a_{1} c^{k_{1}} a_{2} \cdots c^{k_{n-1}} a_{n} c^{k_{n}}$ is nontrivial in $\mathbb{F}$.

Proof of Proposition 2.39 Denote by $\chi(\Sigma)$ and $g(\Sigma)$ the Euler characteristic and the genus of $\Sigma$. We shall work by induction on $(-\chi(\Sigma), g(\Sigma))$, following the lexicographic order.

If $\chi(\Sigma)=-1$, then $\varphi$ is injective (see eg [7, Proposition 3.1]). Hence, suppose that the proposition is true for all $\Sigma^{\prime}$ such that $\left(-\chi\left(\Sigma^{\prime}\right), g\left(\Sigma^{\prime}\right)\right)<(-\chi(\Sigma), g(\Sigma))$ (for the lexicographic order) and consider curves $c_{1}, \ldots, c_{p}$ as in Z Sela's lemma. 
Suppose first that $c_{1}$ is a separating curve: denote $\Sigma=\Sigma_{1} \cup_{c_{1}} \Sigma_{2}$. Put the base point in $\Sigma_{1}$, near $c_{1}$. We have $\pi_{1} \Sigma=\pi_{1} \Sigma_{1} *_{\alpha} \pi_{1} \Sigma_{2}$, where $\alpha$ is represented by the curve $c_{1}$, deformed so that it passes through the base point. Fix a finite subset $P \subset \pi_{1} \Sigma$. For every $m \in P$, choose a writing $m=a_{1} \alpha^{k_{1}} b_{1} \alpha^{l_{1}} \cdots a_{n} \alpha^{k_{n}} b_{n} \alpha^{l_{n}}$, with $a_{i} \in \pi_{1} \Sigma_{1}$ and $b_{i} \in \pi_{1} \Sigma_{2}$, and such that $a_{i}, b_{i}$ do not commute with $\alpha$ (except maybe $a_{1}$ or $b_{n}$, in which case we do not write them in $m$ ). Denote by $P_{1}$ the subset of $\pi_{1} \Sigma_{1}$ defined by the elements $\alpha a_{i} \alpha^{-1} a_{i}^{-1}$ and denote by $P_{2}$ the subset of $\pi_{1} \Sigma_{2}$ defined by the elements $\alpha b_{i} \alpha^{-1} b_{i}^{-1}$. By induction hypothesis, there exists a diffeomorphism $\gamma_{1}$ of $\Sigma_{1}$ fixing the boundary of $\Sigma_{1}$ (as well as the boundary of the curve $c_{1}$ ), and a diffeomorphism $\gamma_{2}$ of $\Sigma_{2}$ fixing the boundary of $\Sigma_{2}$ such that for all $u \in P_{1}$ we have $\varphi \circ \gamma_{1 *}(u) \neq 1$ and such that for all $u \in P_{2}$ we have $\varphi \circ \gamma_{2 *}(u) \neq 1$. Consider then a diffeomorphism $\gamma_{k}: \Sigma \rightarrow \Sigma$ defined by $\gamma_{1}$ on $\Sigma_{1}, \gamma_{2}$ on $\Sigma_{2}$ and by $k$ Dehn twists along $c_{1}$. Then $\gamma_{k *}: \pi_{1} \Sigma \rightarrow \pi_{1} \Sigma$ is defined as follows: if $a_{1}, \ldots, a_{n} \in \pi_{1} \Sigma_{1}$ and $b_{1}, \ldots, b_{n} \in \pi_{1} \Sigma_{2}$, we have

$$
\begin{aligned}
& \gamma_{k *}\left(a_{1} \alpha^{k_{1}} b_{1} \alpha^{l_{1}} \cdots a_{n} \alpha^{k_{n}} b_{n} \alpha^{l_{n}}\right) \\
& \quad=\gamma_{1 *}\left(a_{1}\right) \alpha^{k_{1}+k} \gamma_{2 *}\left(b_{1}\right) \alpha^{l_{1}-k} \cdots \gamma_{1 *}\left(a_{n}\right) \alpha^{k_{n}+k} \gamma_{2 *}\left(b_{1}\right) \alpha^{l_{n}-k} .
\end{aligned}
$$

Let $m \in P$, and consider the expression $m=a_{1} \alpha^{k_{1}} b_{1} \alpha^{l_{1}} \cdots a_{n} \alpha^{k_{n}} b_{n} \alpha^{l_{n}}$ chosen before. We then have

$\varphi \circ \gamma_{k *}(m)=\varphi \circ \gamma_{1 *}\left(a_{1}\right) \varphi(\alpha)^{k_{1}+k} \varphi \circ \gamma_{2 *}\left(b_{1}\right) \varphi(\alpha)^{l_{1}-k}$

$$
\cdots \varphi \circ \gamma_{1 *}\left(a_{n}\right) \varphi(\alpha)^{k_{n}+k} \varphi \circ \gamma_{2 *}\left(b_{n}\right) \varphi(\alpha)^{l_{n}-k} .
$$

Since $\alpha a_{i} \alpha^{-1} a_{i}^{-1} \in P_{1}$, we have $\varphi \circ \gamma_{1 *}\left(\alpha a_{i} \alpha^{-1} a_{i}^{-1}\right) \neq 1$, but $\gamma_{1 *}(\alpha)=\alpha$ : hence $\varphi \circ \gamma_{1 *}\left(a_{i}\right)$ does not commute with $\varphi\left(\alpha_{1}\right)$. All the conditions of G Baumslag's lemma are satisfied, and hence for all $k$ large enough, $\varphi \circ \gamma_{k *}$ sends every nontrivial element of $P$ on a nontrivial element of $\mathbb{F}$.

Suppose finally that $c_{1}$ is a nonseparating curve. Then $\Sigma$, this time, is obtained by gluing two boundary components of a surface $\Sigma_{1}$, and we have $-\chi(\Sigma)=-\chi\left(\Sigma_{1}\right)$ but $g\left(\Sigma_{1}\right)<g(\Sigma)$. We have $\pi_{1} \Sigma=\left\langle\pi_{1} \Sigma_{1}, t \mid t^{-1} \alpha t=\beta\right\rangle$, where $\alpha, \beta \in \pi_{1} \Sigma_{1}$ are represented by the boundary components of $\Sigma_{1}$ concerned by the gluing, and where $t$ is represented, in $\Sigma$, by a simple curve intersecting the curve $c_{1}$ at a single point. The elements $m \in \pi_{1} \Sigma$ can be written (nonuniquely) as $m=t^{k_{0}} a_{1} t^{k_{1}} \cdots a_{n} t^{k_{n}}$ with $a_{i} \in$ $\pi_{1} \Sigma_{1}$; if $\gamma$ is a diffeomorphism of $\Sigma_{1}$ fixing its boundary (as well as a neighborhood of $c_{1}$ containing the base point) then $\gamma_{*}(m)=t^{k_{0}} \gamma_{*}\left(a_{1}\right) t^{k_{1}} \cdots \gamma_{*}\left(a_{n}\right) t^{k_{n}}$, and the image of $m$ under $k$ Dehn twists along $c_{1}$ is equal to $\left(\alpha^{k} t\right)^{k_{0}} a_{1}\left(\alpha^{k} t\right)^{k_{1}} \cdots a_{n}\left(\alpha^{k} t\right)^{k_{n}}$. The same argument as in the preceding case transposes here, thereby finishing the induction. 


\section{Compactifications, degenerations and orientation}

Before going into the study of the compactifications $\overline{m_{g}^{u}}$ and $\overline{m_{g}^{o}}$, we will need to prove a technical fact, namely that the connected components of the spaces $m_{g}^{o}$ and $m_{g}^{u}$ are one-ended. This implies that for every compactification considered, the boundaries of these connected components are connected spaces.

\subsection{The connected components of $m_{g}^{u}$ and $m_{g}^{o}$ are one-ended}

Let us begin with a reminder about topological ends. We refer to Poénaru [38, Chapitre II] for a systematic exposition.

Definition 3.1 Let $X$ be a connected, locally connected, locally compact space. The supremum of the number of unbounded components (ie, whose closure is noncompact) of $X \backslash K$, as $K$ describes the set of compact subsets of $X$, is called the number of ends of $X$.

And of course, if this number is 1 the space $X$ is called one-ended. This notion will be interesting for us for the following reason:

Proposition 3.2 Let $X$ be a one-ended space and let $(\bar{X}, i)$ be a compactification of $X$. Then $\partial \bar{X}$ is connected.

Proof Suppose that $\partial \bar{X}=A \cup B$, where $A$ and $B$ are disjoint, nonempty, open and closed subsets of $\partial \bar{X}$. The boundary $\partial \bar{X}$ being closed, $A$ and $B$ are closed subsets of $\bar{X}$. Since $\bar{X}$ is compact (and Hausdorff, in particular), it is normal. Hence, there exist two open subsets $U$ and $V$ of $\bar{X}$ such that $A \subset U, B \subset V$, and $U \cap V=\varnothing$. The open set $U \cup V$ contains $\partial \bar{X}$ hence it is the complement of a compact subset $K$ of $X$. Now, in $X$, the complement of the compact set $K$ is the open set $(U \backslash A) \cup(V \backslash B)$, and each of the disjoint open sets $U \backslash A$ and $V \backslash B$ are unbounded, hence $X$ has at least two ends.

We will use the following immediate criterium:

Proposition 3.3 Let $X$ be a noncompact, connected, locally connected, locally compact space such that for every compact subset $K$, there exists a compact $K^{\prime}$ such that $K \subset K^{\prime} \subset X$ and such that any two points of $X \backslash K^{\prime}$ are in the same connected component of $X \backslash K$. Then $X$ is one-ended. 
Here, once again, we are interested in the case $\Gamma=\pi_{1} \Sigma_{g}$ and $n=2$; we denote $m_{g}^{o}=m_{\pi_{1} \Sigma_{g}}^{o}(2)$, with the same notation as before. We fix the generating set

$$
S=\left\{a_{1}, a_{1}^{-1}, b_{1}, b_{1}^{-1}, \ldots, b_{g}, b_{g}^{-1}\right\} .
$$

Recall (Corollary 2.14) that $m_{g}^{o}$ and $m_{g}^{u}$ are locally compact and the map d: $m_{g}^{o} \rightarrow \mathbb{R}_{+}$ defined as

$$
\mathbf{d}(\rho)=\min _{x \in \mathbb{H}^{2}} \max _{s \in S} d(x, \rho(s) x)
$$

is continuous and proper. Moreover, $m_{g}^{o}$ and $m_{g}^{u}$ are locally connected (as a real algebraic variety, $R_{g}$ is locally connected, and the projection $R_{g} \rightarrow m_{g}^{o}$ is open by Proposition 2.13).

We are going to prove that the connected components of $m_{g}^{o}$, as well as those of $m_{g}^{u}$, are one-ended. Since the proof is exactly the same in both cases, until the end of this section (and only in this section) we will denote by $m_{g}$ these representation spaces, and by $m_{g, k}$ the corresponding connected components, being vague whether we consider the oriented or the unoriented representation space.

In [23], N Hitchin proved that for all $k \in\{1, \ldots, 2 g-2\}$, the connected component $m_{g, k}$ of $m_{g}$ is homeomorphic to a complex vector bundle of dimension $g-1+|k|$ on the $(2 g-2-|k|)-$ th symmetric product of the surface. It follows, in particular, that the connected component $m_{g, k}$ is one-ended, for every $k \neq 0$. We shall give a much more elementary proof (than the one of [23]) of this result, and generalise it to the case $k=0$.

Proposition 3.4 For all $g \geq 2$, and all $k$ such that $|k| \leq 2 g-2$, the space $m_{g, k}$ is one-ended.

First let us fix some notation. If $S^{\prime} \subset S$, we denote

$$
\mathbf{d}_{S^{\prime}}([\rho])=\inf _{x \in \mathbb{H}^{2}} \max _{s \in S^{\prime}} d(x, \rho(s) x) .
$$

If $r \geq 0$ and $S^{\prime} \subset S$, we denote

$$
K_{S^{\prime}}^{r}=\left\{[\rho] \in m_{g} \mid \mathbf{d}_{S^{\prime}}([\rho]) \leq r\right\}, \quad K^{r}=K_{S}^{r}, \quad U_{S^{\prime}}^{r}=m_{g} \backslash K_{S^{\prime}}^{r}
$$

If $s \in S, U_{\{s\}}^{r}$ is simply the set of conjugacy classes of representations $\rho$ such that $\rho(s)$ is a hyperbolic element whose translation length is strictly greater than $r$, which is equivalent to $\operatorname{Tr}(\rho(s))>2 \cosh r$. Of course, representations can go to infinity without even leaving $\bigcap_{s \in S} K_{\{s\}}^{0}$ : traces of single generators do not say much about representations going to infinity. However, it suffices to consider pairs of generators: 
Lemma 3.5 Let $[\rho] \in m_{g} \backslash K^{r+4 g \delta\left(\mathbb{H}^{2}\right)}$. Then there exist $s_{1}, s_{2} \in\left\{a_{1}, \ldots, b_{g}\right\}$ such that $\mathbf{d}_{\left\{s_{1}, s_{2}\right\}}([\rho])>r$.

Recall that by $\delta\left(\mathbb{H}^{2}\right)$ we mean the best constant of Gromov hyperbolicity of $\mathbb{H}^{2}$. There are several equivalent definitions of the Gromov hyperbolicity (see eg Papadopoulos [8, Chapitre 1] or Ghys and de la Harpe [17, Chapitre 2]), and in all this text we say that a geodesic metric space $X$ is $\delta$-hyperbolic if, for every geodesic triangle, there exists a point at distance at most $\delta$ from each side of the triangle.

Proof If $\rho \in R_{g}, S^{\prime} \subset S$ and $\alpha>0$, let $F_{S^{\prime}}^{\alpha}(\rho)=\left\{x \in \mathbb{H}^{2} \mid \forall s \in S^{\prime}, d(x, \rho(s) x) \leq \alpha\right\}$. Let $\alpha>0$, and suppose that $F_{\left\{s_{1}, s_{2}\right\}}^{\alpha}(\rho) \neq \varnothing$ for every pair $\left\{s_{1}, s_{2}\right\} \subset\left\{a_{1}, \ldots, b_{g}\right\}$. Then for every triple $\left\{s_{1}, s_{2}, s_{3}\right\}$, the convex sets $F_{\left\{s_{1}\right\}}^{\alpha}(\rho), F_{\left\{s_{2}\right\}}^{\alpha}(\rho), F_{\left\{s_{3}\right\}}^{\alpha}(\rho)$ intersect pairwise, hence, since $\mathbb{H}^{2}$ is $\delta\left(\mathbb{H}^{2}\right)$-hyperbolic, there exists a point $x$ at distance at most $\delta\left(\mathbb{H}^{2}\right)$ of each of these three sets. This implies that

$$
x \in F_{\left\{s_{1}, s_{2}, s_{3}\right\}}^{\alpha+2 \delta\left(\mathbb{H}^{2}\right)}(\rho) .
$$

More generally, if $u>0$ is such that for every $k$-tuple $S^{\prime}$ of $\left\{a_{1}, \ldots, b_{g}\right\}, F_{S^{\prime}}^{u} \neq \varnothing$, then, if $\left\{s_{1}, \ldots, s_{k+1}\right\} \subset\left\{a_{1}, \ldots, b_{g}\right\}$, the convex sets

$$
F_{\left\{s_{1}, \ldots, s_{k-1}\right\}}^{u}(\rho), \quad F_{\left\{s_{1}, \ldots, s_{k-2}, s_{k}\right\}}^{u}(\rho) \quad \text { and } \quad F_{\left\{s_{1}, \ldots, s_{k-2}, s_{k+1}\right\}}^{u}(\rho)
$$

intersect pairwise, and it follows that

$$
F_{\left\{s_{1}, \ldots, s_{k+1}\right\}}^{u+2 \delta\left(\mathbb{H}^{2}\right)}(\rho) \neq \varnothing .
$$

Therefore, by induction on $\operatorname{Card}\left\{a_{3}, \ldots, b_{g}\right\}$, we get that $\mathrm{d}(\rho) \leq \alpha+4 g \delta\left(\mathbb{H}^{2}\right)$, as soon as $F_{\left\{s_{1}, s_{2}\right\}}^{\alpha}(\rho) \neq \varnothing$ for every pair $\left\{s_{1}, s_{2}\right\} \subset\left\{a_{1}, \ldots, b_{g}\right\}$.

Now if $[\rho] \in m_{g} \backslash K^{r+4 g \delta\left(\mathbb{H}^{2}\right)}$, then there exists $\varepsilon>0$ such that $d(\rho)>r+4 g \delta\left(\mathbb{H}^{2}\right)+\varepsilon$, which implies that there exists a pair $\left\{s_{1}, s_{2}\right\} \subset\left\{a_{1}, \ldots, b_{g}\right\}$ such that $F_{\left\{s_{1}, s_{2}\right\}}^{r+\varepsilon}(\rho)=\varnothing$; this implies that $\mathrm{d}_{\left\{s_{1}, s_{2}\right\}}(\rho) \geq r+\varepsilon$ hence $\mathrm{d}_{\left\{s_{1}, s_{2}\right\}}(\rho)>r$.

For the proof of Proposition 3.4, it will be useful to write explicit deformations of representations, which enable to go to infinity in the space of representations. For all $A \in \operatorname{PSL}(2, \mathbb{R})$, we wish to choose a one-parameter subgroup $\left(A_{t}\right)_{t \in \mathbb{R}}$ passing through $A$. If $A=\mathrm{Id}$, we set $A_{t}=\mathrm{Id}$, for all $t \in \mathbb{R}$. If $A$ is parabolic or hyperbolic, we choose $A_{t}$ such that $A_{0}=\mathrm{Id}$ and $A_{1}=A$. If $A$ is elliptic and different from the identity, we further require that $A_{t} \neq \mathrm{Id}$ for all $t \in(0,1]$, and that $A_{t}$ is a rotation of positive angle for small $t$. Note that for all $n \in \mathbb{Z}$, we have $A_{n}=A^{n}$.

Lemma 3.6 Let $A, B \in \operatorname{PSL}(2, \mathbb{R})$ such that $[A, B] \neq \mathrm{Id}$. Then for all $r>0$, there exist $x \in \mathbb{R}$ and $n \in \mathbb{Z}$ such that at least one of the following holds: $\operatorname{Tr}\left(A \cdot\left(B A_{x}\right)^{n}\right)>$ $2 \cosh (r)$, or $\operatorname{Tr}\left(B \cdot\left(A B_{x}\right)^{n}\right)>2 \cosh (r)$. 
Proof Suppose first that $A$ is hyperbolic. Up to conjugating simultaneously $A$ and $B$, we have

and

$$
\begin{aligned}
A & =\left(\begin{array}{cc}
\lambda & 0 \\
0 & 1 / \lambda
\end{array}\right) \quad \text { with } \lambda>1 \\
B & =\left(\begin{array}{ll}
a & b \\
c & d
\end{array}\right) .
\end{aligned}
$$

If $a \neq 0$ or $d \neq 0$, then $\operatorname{Tr}\left(B \cdot\left(A B_{x}\right)^{n}\right)$ is as large as we need, provided $|n|$ is large enough (and $x=0$ ). The condition $a=d=0$ means that $B$ is an elliptic element of order 2 whose fixed point lies on the axis of $A$. In that case, if $x$ is small, then $A \cdot B_{x}$ is still a hyperbolic element, whose axis does not contain the fixed point of $B$ any more (this can be seen easily by decomposing $B_{x}$ and $A$ as products of two reflections). Obviously this also deals with the case when $B$ is hyperbolic.

If none of $A$ and $B$ is hyperbolic but, say, $A$ is parabolic, then up to conjugation,

$$
A=\left(\begin{array}{ll}
1 & 1 \\
0 & 1
\end{array}\right) \quad \text { and } \quad B=\left(\begin{array}{ll}
a & b \\
c & d
\end{array}\right) .
$$

Now, with $n=1$ and $x$ large enough, $\operatorname{Tr}\left(A \cdot\left(B A_{x}\right)^{n}\right)$ is as large as we need, provided $x$ is large enough, except possibly if $c=0$ : but this would imply $[A, B]=\operatorname{Id}$.

We are left with the case when $A, B$ are elliptic, with distinct fixed points. If $\ell$ is the geodesic line joining these two points, $A_{x}$ (resp. $B$ ) is the composition of the reflection with respect to a line $\ell_{A_{x}}$ (resp. $\ell_{B}$ ) and the reflection with respect to $\ell$. For a suitable $x$, the lines $\ell_{A_{x}}$ and $\ell_{B}$ do not intersect in $\overline{\mathbb{H}^{2}}$, hence $B A_{x}$ is a hyperbolic element whose axis does not contain the fixed point of $A$. Thus, for $|n|$ sufficiently large, $\operatorname{Tr}\left(A \cdot\left(B A_{x}\right)^{n}\right)$ is as large as needed.

Note that for all $x, y \in \mathbb{R},[A, B]=\left[A B_{x}, B \cdot\left(A B_{x}\right)_{y}\right]=\left[A \cdot\left(B A_{x}\right)_{y}, B A_{x}\right]$ : we have deformed $A$ and $B$ without changing their commutator. Hence, if $\rho \in R_{g}$, we may define $\varphi_{i, x, y}(\rho) \in R_{g}$ by setting $\varphi_{i, x, y}(\rho)\left(a_{j}\right)=\rho\left(a_{j}\right)$ and $\varphi_{i, x, y}(\rho)\left(b_{j}\right)=\rho\left(b_{j}\right)$ for $j \neq i$, and $\varphi_{i, x, y}(\rho)\left(a_{i}\right)=\rho\left(a_{i}\right) \rho\left(b_{i}\right)_{x}, \varphi_{i, x, y}(\rho)\left(b_{i}\right)=\rho\left(b_{i}\right) \cdot\left(\rho\left(a_{i}\right) \rho\left(b_{i}\right)_{x}\right)_{y}$, and, similarly, define a deformation $\psi_{i, x, y}$ by using the other deformation of the commutator $\left[\rho\left(a_{i}\right), \rho\left(b_{i}\right)\right]$. Note that we always have $\varphi_{i, 0,0}(\rho)=\psi_{i, 0,0}(\rho)=\rho$.

Now we can prove that the connected components of $m_{g}$ are one-ended.

Proof of Proposition 3.4 We will use the criterium given by Proposition 3.3, and prove that for every $k \in \mathbb{Z}$ such that $|k| \leq 2 g-2$, and all $r>6 \delta\left(\mathbb{H}^{2}\right)$, any two representations $\rho, \rho^{\prime} \in m_{g, k} \backslash K^{r+4 g \delta\left(\mathbb{H}^{2}\right)}$ can be joined by a path in $m_{g, k} \backslash K^{r}$. 
The requirement that $r \geq 6 \delta\left(\mathbb{H}^{2}\right)$ is technical and will be used only at the end of this proof, in the case when $g=2$.

Step 1 Let $\rho_{0}, \rho_{1} \in m_{g, k} \cap U_{\left\{a_{1}\right\}}^{r} \cap U_{\left\{a_{2}\right\}}^{r}$. Then there is a path $\rho_{t} \in \bigcup_{s \in\left\{a_{1}, b_{1}, a_{2}, b_{2}\right\}} U_{\{s\}}^{r}$ joining $\rho_{0}$ and $\rho_{1}$.

By Lemma 10.1 of Goldman [20], for every $k \in \mathbb{Z}$ such that $|k| \leq 2 g-2$, the set of representations $\rho$ such that $\left[\rho\left(a_{i}\right), \rho\left(b_{i}\right)\right] \neq \mathrm{Id}$ is path-connected and dense in $m_{g, k}$. We can thus perturb $\rho_{0}$ and $\rho_{1}$, and find a path $\rho_{t} \in m_{g, k}$ joining $\rho_{0}$ to $\rho_{1}$ and such that for all $t \in[0,1]$ and all $i \in\{1, \ldots, g\},\left[\rho_{t}\left(a_{i}\right), \rho_{t}\left(b_{i}\right)\right] \neq \mathrm{Id}$.

Let $t \in[0,1]$ and $i \in\{1,2\}$. By Lemma 3.6, there exist $y_{i}(t) \in \mathbb{Z}$ and $x_{i}(t) \in \mathbb{R}$ such that

$$
\operatorname{Tr}\left(\psi_{i, x_{i}(t), y_{i}(t)}\left(\rho_{t}\right)\left(a_{i}\right)\right)>2 \cosh r, \text { or } \operatorname{Tr}\left(\varphi_{i, x_{i}(t), y_{i}(t)}\left(\rho_{t}\right)\left(b_{i}\right)\right)>2 \cosh r .
$$

These inequalities being strict, for all $\tau$ there exists an interval $(\tau-\delta, \tau+\delta)$ such that for all $t \in(\tau-\delta, \tau+\delta) \cap[0,1]$, we still have

$$
\operatorname{Tr}\left(\psi_{i, x_{i}(\tau), y_{i}(\tau)}\left(\rho_{t}\right)\left(a_{i}\right)\right)>2 \cosh r, \text { or } \operatorname{Tr}\left(\varphi_{i, x_{i}(\tau), y_{i}(\tau)}\left(\rho_{t}\right)\left(b_{i}\right)\right)>2 \cosh r .
$$

The compact set $[0,1]$ is covered by finitely many such intervals, hence there exists a subdivision $0=t_{0}<t_{1}<\cdots<t_{k}=1$, and elements $x_{i}^{j}, y_{i}^{j} \in R$ such that for every $i \in\{1,2\}$ and $j \in\{0, \ldots, k-1\}$ and for all $t \in\left[t_{j}, t_{j+1}\right]$ we have

$$
\operatorname{Tr}\left(\psi_{i, x_{i}\left(t_{j}\right), y_{i}\left(t_{j}\right)}\left(\rho_{t}\right)\left(a_{i}\right)\right)>2 \cosh r, \text { or } \operatorname{Tr}\left(\varphi_{i, x_{i}\left(t_{j}\right), y_{i}\left(t_{j}\right)}\left(\rho_{t}\right)\left(b_{i}\right)\right)>2 \cosh r .
$$

For simplicity, we will suppose that all the relevant deformations are the $\varphi$ 's.

Now we can construct a path joining $\rho_{0}$ to $\rho_{1}$ as follows. Start with $\rho_{0}$. Let $(x, y)$ go from $(0,0)$ to $\left(x_{1}(0), y_{1}(0)\right)$ to define a path $\psi_{1, x, y}\left(\rho_{0}\right)$; this path does not leave $U_{\left\{a_{2}\right\}}^{r}$. Then go to $\psi_{2, x_{2}(0), y_{2}(0)}\left(\psi_{1, x_{1}(0), y_{1}(0)}(\rho)\right)$, similarly, without leaving $U_{\left\{b_{1}\right\}}^{r}$. Then let $t$ vary from $0=t_{0}$ to $t_{1}$ along the path $\psi_{2, x_{2}(0), y_{2}(0)}\left(\psi_{1, x_{1}(0), y_{1}(0)}(\rho)\right)$ which does not leave $U_{\left\{b_{2}\right\}}^{r}$. Then let the indices $\left(x_{1}, y_{1}\right)$ vary from $\left(x_{1}(0), y_{1}(0)\right)$ to $\left(x_{1}\left(t_{1}\right), y_{1}\left(t_{1}\right)\right)$ without leaving $U_{\left\{b_{2}\right\}}^{r}$, then we deal with the indices $\left(x_{2}, y_{2}\right)$, and so on. This finishes Step 1.

Step 2 Let $\rho \in m_{g, k} \backslash K^{r+4 g \delta\left(\mathbb{H}^{2}\right)}$. Then there exists a path $\rho_{t}$ taking values in $m_{g, k} \backslash K^{r}$, such that $\rho_{0}=\rho$ and $\rho_{1} \in U_{\left\{a_{1}\right\}}^{r} \cap U_{\left\{a_{2}\right\}}^{r}$.

By Lemma 3.5, there exist $s_{1}, s_{2} \in\left\{a_{1}, \ldots, b_{g}\right\}$ such that $F_{\left\{s_{1}, s_{2}\right\}}^{r}(\rho) \neq \varnothing$. If $g \geq 3$, then there exists $i \in\{1, \ldots, g\}$ such that $\left\{a_{i}, b_{i}\right\} \cap\left\{s_{1}, s_{2}\right\}=\varnothing$. In that case, as in Step 1 , we can deform the handle $i$, without entering $K^{r}$ since we do not touch $\rho\left(s_{1}\right)$, $\rho\left(s_{2}\right)$. Then we can deform the handles 1 and 2 (or just one, if $i \in\{1,2\}$ ) without 
entering $K^{r}$ because of the handle $i$. This completes the proof of Proposition 3.4, in the case $g \geq 3$.

In the case when $g=2$ and $\left\{s_{1}, s_{2}\right\}=\left\{a_{1}, b_{1}\right\}$ or $\left\{s_{1}, s_{2}\right\}=\left\{a_{2}, b_{2}\right\}$, we do the same as in the preceding case. Now suppose for instance that $s_{1}=a_{1}$ and $s_{2}=a_{2}$ (the other cases are dealt with similarly: the roles of $a_{i}$ and $b_{i}$ are always symmetric). If $\rho\left(a_{1}\right)$ or $\rho\left(a_{2}\right)$ is hyperbolic (say, for instance, $\rho\left(a_{1}\right)$ ), then, exactly as in the proof of Lemma 3.6, we can deform the handle 1 so that $\operatorname{Tr}\left(\rho_{t}\left(b_{1}\right)\right)>2 \cosh r$ (almost) without touching $\rho\left(a_{1}\right)$, and leaving $\rho\left(a_{2}\right)$ unchanged. Otherwise, if $\rho\left(a_{1}\right)$ or $\rho\left(a_{2}\right)$ is parabolic (say, $\left.\rho\left(a_{1}\right)\right)$, and if $\left[\rho\left(a_{1}\right), \rho\left(b_{1}\right)\right] \neq$ Id then for $x$ large enough, $\operatorname{Tr}\left(\rho\left(b_{1}\right) \rho\left(a_{1}\right)_{x}\right)$ can be made as large as needed (bigger than $2 \cosh (r)$ ), providing a deformation in the first handle as desired.

The only case left is when $\rho\left(a_{1}\right)$ and $\rho\left(a_{2}\right)$ are elliptic. Note that $\rho\left(a_{1} a_{2}\right)$ has to be hyperbolic in this case: indeed, let $\ell$ be the line joining the fixed points of $\rho\left(a_{1}\right)$ and $\rho\left(a_{2}\right)$, and suppose that $\rho\left(a_{1}\right)$ is the composition of the reflection along a line $\ell_{1}$ and the reflection along $\ell$, and that $\rho\left(a_{2}\right)$ is the composition of the reflection along $\ell$ and the reflection along a line $\ell_{2}$. If $\ell_{1}$ and $\ell_{2}$ were allowed to meet in $\overline{\mathbb{H}^{2}}$, there would be a point at distance at most $\delta\left(\mathbb{H}^{2}\right)$ of each of these three lines, and this point would be moved by less than $6 \delta\left(\mathbb{H}^{2}\right)$ by $\rho\left(a_{1}\right)$ and $\rho\left(a_{2}\right)$. This time, instead of using continuous Dehn twists inside the handles, we are going to do one along the curve freely homotopic to $a_{1} a_{2}$. Let us define $\phi_{x}(\rho)$ as follows:

$$
\begin{array}{ll}
\phi_{x}(\rho)\left(a_{1}\right)=\rho\left(a_{1}\right), & \phi_{x}(\rho)\left(b_{1}\right)=\left(\rho\left(a_{2}\right) \rho\left(a_{1}\right)\right)_{x} \rho\left(b_{1}\right), \\
\phi_{x}(\rho)\left(a_{2}\right)=\rho\left(a_{2}\right), & \phi_{x}(\rho)\left(b_{2}\right)=\left(\rho\left(a_{1}\right) \rho\left(a_{2}\right)\right)_{x} \rho\left(b_{2}\right) .
\end{array}
$$

We need to check that $\phi_{x}$ defines indeed a morphism from $\pi_{1} \Sigma_{2}$ in $\operatorname{PSL}(2, \mathbb{R})$. This amounts exactly to check that for all $x \in \mathbb{R}, \rho\left(a_{1}\right) \cdot\left(\rho\left(a_{2}\right) \rho\left(a_{1}\right)\right)_{x} \rho\left(a_{1}\right)^{-1}=$ $\left(\rho\left(a_{1}\right) \rho\left(a_{2}\right)\right)_{x}$. This is clearly true when $x$ is an integer, hence $\rho\left(a_{1}\right)$ maps the oriented axis of the hyperbolic isometry $\left(\rho\left(a_{1}\right) \rho\left(a_{2}\right)\right)_{x}$ (this is valid for all $x$ ) to the one of $\left(\rho\left(a_{2}\right) \rho\left(a_{1}\right)\right)_{x}$; and these two hyperbolic isometries have the same translation length: hence the desired relation indeed holds for all $x$. Now, the deformation $x \mapsto \phi_{x}(\rho)$ does not change $\rho\left(a_{1}\right)$ or $\rho\left(a_{2}\right)$, hence $\phi_{x}(\rho)$ stays in $m_{g, k} \backslash K^{r}$. And for $x$ large enough, $\operatorname{Tr}\left(\rho\left(a_{2} a_{1}\right)_{x} \rho\left(b_{1}\right)\right)$ can be made as large as we want, except if $\rho\left(b_{1}\right)$ is elliptic of order two with its fixed point on the axis of $\rho\left(a_{2} a_{1}\right)$, but this last accident can be avoided by first replacing $\rho\left(b_{1}\right)$ by $\rho\left(b_{1}\right) \rho\left(a_{1}\right)$ y for a suitable $y \in \mathbb{R}$. We conclude as in the preceding cases.

Similarly, we can prove that every loop can be pushed out of every compact set. In other words, the fundamental group of the space $e^{-1}(k)$ is entirely carried by this only end. 


\subsection{Oriented compactification}

In what follows we will mostly consider the case $n=2$, and consider the compactification of the space $m_{\Gamma}^{u}$ of actions on the hyperbolic plane $\mathbb{H}^{2}$. We will prove, at least in the case when $\Gamma$ is a surface group, that this compactification has quite a wild behaviour; and it will seem more natural to study a compactification of the oriented version $m_{\Gamma}^{o}$.

As we said before, the ideal points of the compactification $\overline{m_{g}^{u}}$ of M Bestvina and F Paulin are (equivariant isometry classes of) actions of $\pi_{1} \Sigma_{g}$ by isometries on $\mathbb{R}$ trees. We shall prove that it is possible to equip these $\mathbb{R}$-trees with an orientation; this will enable us to define an Euler class on these trees, and to define a compactification of $m_{g}^{o}$, in which the Euler class extends continuously to the boundary.

3.2.1 Oriented $\mathbb{R}$-trees Let $X$ be a hyperbolic space in the sense of Gromov. A germ of oriented segments, in $X$, is an equivalence class of nondegenerate oriented segments, for the following equivalence relation: we say that two oriented segments are equivalent if they coincide on some nontrivial initial segment.

Let $T$ be an $\mathbb{R}$-tree nonreduced to a point. At every point $x \in T$, denote by $\mathcal{G}(x)$ the set of germs of oriented segments starting at $x$. An orientation of $T$ is the data, for all $x \in T$, of a total cyclic order $\operatorname{or}(x)$ in $\mathcal{G}(x)$. The set of orientations on $T$ will be denoted by $\operatorname{Or}(T)$.

Definition 3.7 - An $\mathbb{R}$-tree equipped with an orientation is called an oriented $\mathbb{R}$-tree.

- Let $\left(T\right.$, or) and $\left(T^{\prime}\right.$, or $\left.^{\prime}\right)$ be two oriented $\mathbb{R}$-trees and let $h \in \operatorname{Isom}\left(T, T^{\prime}\right)$ be an isometry. Of course, $h$ defines, at every point $x \in T$, a bijection $\mathcal{G} h_{x}: \mathcal{G}(x) \rightarrow$ $\mathcal{G}(h(x))$. We say that $h$ preserves the orientation if for every $x \in T$, the following diagram commutes:

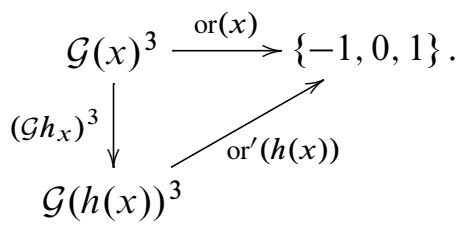

The set of isometries of $T$ which preserve the orientation or form a subgroup of $\operatorname{Isom}(T)$, which will be denoted by $\operatorname{Isom}^{\text {or }}(T)$.

- We say that an action $\rho: \Gamma \rightarrow \operatorname{Isom}(X)$ of a group $\Gamma$ by isometries preserves the orientation or if it takes values into $\operatorname{Isom}^{\text {or }}(T)$. 
Since we are interested in defining the Euler class of actions on $\mathbb{R}$-trees preserving the orientation, we need to consider more particularly the boundary of the tree. Note that, if $r$ is a ray in a tree, its initial segments define a germ of oriented segments, and a germ of rays in $T$ will be an equivalence class of rays, for the relation of defining the same germ of oriented segment. We say that a total cyclic order $\mathbf{o}$ on $\partial_{\infty} T$ is coherent if for every $x \in T$ and every nondegenerate triple $\left(\left[r_{1}\right],\left[r_{2}\right],\left[r_{3}\right]\right)$ of germs rays starting at $x$, the element $\mathbf{o}\left(r_{1}, r_{2}, r_{3}\right)$ does not depend on the chosen representatives $r_{1}, r_{2}, r_{3}$ of $\left[r_{1}\right],\left[r_{2}\right],\left[r_{3}\right]$. For instance, in the following configuration

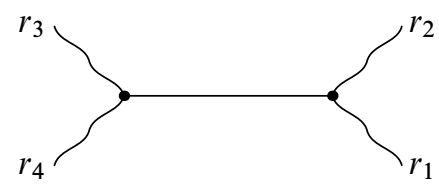

a total cyclic order $\mathbf{o}$ on the boundary $\left\{r_{1}, r_{2}, r_{3}, r_{4}\right\}$ is coherent if and only if it satisfies $\mathbf{o}\left(r_{1}, r_{2}, r_{3}\right)=\mathbf{o}\left(r_{1}, r_{2}, r_{4}\right)$ and $\mathbf{o}\left(r_{1}, r_{3}, r_{4}\right)=\mathbf{o}\left(r_{2}, r_{3}, r_{4}\right)$.

Under natural conditions, there is an identification between orientations on an $\mathbb{R}-$ tree and coherent total cyclic orders on its boundary at infinity. Let us begin with some notation. Borrowing the terminology of B Leeb [26], we say that an $\mathbb{R}$-tree has extendible segments if every segment is contained in a complete geodesic (ie, a geodesic isometric to $\mathbb{R}$ ). Equivalently, every oriented segment is the initial segment of some ray.

If $x \in T$, let $\operatorname{Trip}(x)$ denote the set of nondegenerate triples of germs of oriented segments starting at $x$. The set of all elements of $\operatorname{Trip}(x)$, as $x$ describes $T$, will be denoted by $\operatorname{Trip}(T)$. If $a_{0} \in T$, and if the oriented segments $\left[a_{0}, a_{1}\right],\left[a_{0}, a_{2}\right],\left[a_{0}, a_{3}\right]$ define three pairwise distinct germs of rays starting at $a_{0}$, the corresponding element in $\operatorname{Trip}\left(a_{0}\right)$ will be denoted by $\operatorname{Trip}\left(a_{0}, a_{1}, a_{2}, a_{3}\right)$. Such elements will be called germs of tripods of $T$. With this notation, an orientation of $T$ is simply a function from $\operatorname{Trip}(T)$ to $\{-1,1\}$ satisfying the following conditions (see Remark 2.22):

$$
\begin{gathered}
\operatorname{or}\left(\operatorname{Trip}\left(a_{0}, a_{1}, a_{2}, a_{3}\right)\right)=\operatorname{or}\left(\operatorname{Trip}\left(a_{0}, a_{2}, a_{3}, a_{1}\right)\right)=-\operatorname{or}\left(\operatorname{Trip}\left(a_{0}, a_{1}, a_{3}, a_{2}\right)\right) \\
\left\{\begin{aligned}
& \operatorname{or}\left(\operatorname{Trip}\left(a_{0}, a_{1}, a_{2}, a_{3}\right)\right) \operatorname{or}\left(\operatorname{Trip}\left(a_{0}, a_{1}, a_{3}, a_{4}\right)\right)=1 \\
& \Rightarrow \operatorname{or}\left(\operatorname{Trip}\left(a_{0}, a_{1}, a_{2}, a_{4}\right)\right)=1 .
\end{aligned}\right.
\end{gathered}
$$

Let us denote by $\operatorname{Preor}(T)$ the set of all functions $\operatorname{Trip}(T) \rightarrow\{-1,1\}$ and equip it with the product topology. By Tikhonov's theorem, it is a compact space. The conditions (3-1) and (3-2) being closed, $\operatorname{Or}(T)$, equipped with the induced topology, is compact.

In an $\mathbb{R}$-tree with extendible segments, there is a natural identification between the set of orientations, $\operatorname{Or}(T)$, and the set of coherent cyclic orders on $\partial_{\infty} T$. Let us denote 
by $\operatorname{Preo}\left(\partial_{\infty} T\right)$ the set of all functions $\left(\partial_{\infty} T\right)^{3} \rightarrow\{-1,0,1\}$. Then the set of coherent cyclic orders on $T$ is the subset $\mathcal{O}\left(\partial_{\infty} T\right)$ of functions satisfying the conditions of Definition 2.20 and the coherence condition.

For every nondegenerate triple $(x, y, z) \in\left(\partial_{\infty} T\right)^{3}$, the intersection $(x, y) \cap(y, z) \cap$ $(x, z)$ is a point, which will be denoted by $P_{x y z}$. Given an orientation or $\operatorname{Or}(T)$, and a triple $(x, y, z) \in\left(\partial_{\infty} T\right)^{3}$, we can set Push(or) $(x, y, z)=0$ if $\operatorname{Card}\{x, y, z\} \leq 2$ and otherwise, $\operatorname{Push}($ or $\left.)(x, y, z)=\operatorname{or}\left(\left[P_{x y z}, x\right),\left[P_{x y z}, y\right), P_{x y z}, z\right)\right)$, with a slight abuse of notation (we have written rays instead of the germs of initial segments they define). This defines a map Push: $\operatorname{Or}(T) \rightarrow \operatorname{Preo}\left(\partial_{\infty} T\right)$.

Proposition 3.8 Let $T$ be an $\mathbb{R}$-tree with extendible geodesics. Then Push induces a bijection $\operatorname{Or}(T) \rightarrow \mathcal{O}\left(\partial_{\infty} T\right)$.

And of course, if we equip Preo( $T)$ with the product topology, this bijection becomes a homeomorphism.

Proof First let us check that Push has image in $\mathcal{O}\left(\partial_{\infty} T\right)$. Let or $\in \operatorname{Or}(T)$, and $\mathbf{o}=\operatorname{Push}$ (or). It follows directly from the construction of Push that $\mathbf{o}$ satisfies the conditions (i) and (ii) of Definition 2.20, as well as the coherence condition. Now let $r_{1}, r_{2}, r_{3}, r_{4} \in \partial_{\infty} T$ be such that $\mathbf{o}\left(r_{1}, r_{2}, r_{3}\right)=\mathbf{o}\left(r_{1}, r_{3}, r_{4}\right)=1$, we need to prove that $\mathbf{o}\left(r_{1}, r_{2}, r_{4}\right)=1$. If $P_{r_{1} r_{3} r_{4}} \in\left(r_{1}, P_{r_{1} r_{2} r_{3}}\right)$, then

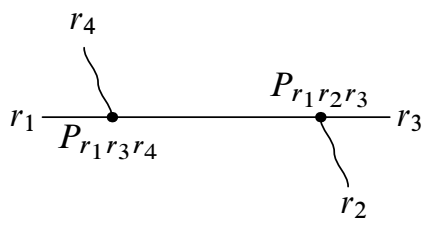

$P_{r_{1} r_{2} r_{4}}=P_{r_{1} r_{3} r_{4}}$ and the germs of $\left[P_{r_{1} r_{2} r_{4}}, r_{2}\right)$ and $\left[P_{r_{1} r_{2} r_{4}}, r_{3}\right)$ are identical, so that $\mathbf{o}\left(r_{1}, r_{2}, r_{4}\right)=1$. If $P_{r_{1} r_{3} r_{4}} \in\left(P_{r_{1} r_{2} r_{3}}, r_{3}\right)$ the argument is similar. If $P_{r_{1} r_{3} r_{4}}=$ $P_{r_{1} r_{2} r_{3}}$, then the germs of the rays $\left[P_{r_{1} r_{2} r_{3}}, r_{2}\right)$ and $\left[P_{r_{1} r_{2} r_{3}}, r_{4}\right)$ are distinct since

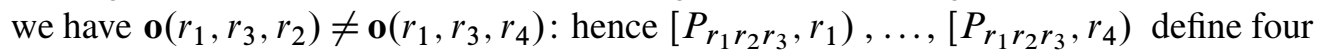
pairwise distinct germs of rays issued from $P_{r_{1}} r_{2} r_{3}$, and we have $\mathbf{o}\left(r_{1}, r_{2}, r_{4}\right)=1$ because or satisfies condition (3-2).

Now, using the assumption that $T$ has extendible geodesics, given an element $\mathbf{o} \in$ $\mathcal{O}\left(\partial_{\infty} T\right)$, if Trip $\left(a_{0}, a_{1}, a_{2}, a_{3}\right)$ is a germ of tripods of $T$, we can extend the oriented segments $\left[a_{0}, a_{1}\right],\left[a_{0}, a_{2}\right]$ and $\left[a_{0}, a_{3}\right]$ to rays $r_{1}, r_{2}$ and $r_{3}$ starting at $a_{0}$, and define $\operatorname{Push}^{-1}\left(\mathbf{o}\left(\operatorname{Trip}\left(a_{0}, a_{1}, a_{2}, a_{3}\right)\right)\right)=\mathbf{o}\left(r_{1}, r_{2}, r_{3}\right)$. This function $\operatorname{Push}^{-1}(\mathbf{o})$ obviously satisfies the conditions (3-1) and (3-2), and by constructions, Push and $\mathrm{Push}^{-1}$ are inverse bijections. 
Now recall that an action of a group $\Gamma$ by isometries on an $\mathbb{R}$-tree $T$ is called minimal if $T$ possesses no subtree $T^{\prime} \subset T$, invariant under the action of $\Gamma$, distinct from $\varnothing$ and $T$.

In the sequel, $\Gamma$ is a finitely generated group and we consider minimal actions of $\Gamma$ on $\mathbb{R}$-trees. In that case, $T$ is the union of the translation axes of the hyperbolic elements in the image of $\Gamma$ (see eg $[32 ; 36]$ ). In particular, such trees have extendible segments (this is Lemma 4.3 of [36]).

Also, we will need to consider the set of classes of minimal actions of $\Gamma$ on oriented $\mathbb{R}$-trees, preserving the orientation, up to equivariant isometry preserving the order. General arguments of cardinality enable to do that, but the following proposition enables us to see this as an explicit set.

If ( $T$, or) is an oriented $\mathbb{R}$-tree and if $u, v, w \in T$ are not aligned, then they define a class of tripods denoted by $\operatorname{Trip}(u, v, w)$, and we denote by $o(u, v, w) \in\{-1,1\}$ the image of this tripod by or. If $u, v, w$ are aligned, then we write $o(u, v, w)=0$ (only in this section). We will come back to this notation in the following section.

Proposition 3.9 Let $\left(T\right.$, or) be an oriented $\mathbb{R}$-tree, let $x_{0} \in T$, and let $\rho: \Gamma \rightarrow$ $\operatorname{Isom}^{\text {or }}(T)$ be a minimal action of a finitely generated group $\Gamma$, preserving the orientation. Then the $\mathbb{R}$-tree $T$, the orientation or and the action $\rho$ are entirely determined by the functions $f: \Gamma^{2} \rightarrow \mathbb{R}$ and $g: \Gamma^{3} \rightarrow\{-1,0,1\}$ defined by

$$
f\left(\gamma_{1}, \gamma_{2}\right)=d_{T}\left(\gamma_{1} x_{0}, \gamma_{2} x_{0}\right) \text { and } g\left(\gamma_{1}, \gamma_{2}, \gamma_{3}\right)=o\left(\gamma_{1} x_{0}, \gamma_{2} x_{0}, \gamma_{3} x_{0}\right) .
$$

More precisely, if $\rho: \Gamma \rightarrow \operatorname{Isom}^{\text {or }}(T)$ and $\rho^{\prime}: \Gamma \rightarrow \operatorname{Isom}^{\text {or }^{\prime}}\left(T^{\prime}\right)$ define the same functions $f$ and $g$, for some choices of base points in $T$ and $T^{\prime}$, then there exists an equivariant isometry $\varphi: T \rightarrow T^{\prime}$ preserving the order and the base point.

Proof This is well-known (see eg [36]) for minimal actions of a finitely generated groups on $\mathbb{R}$-trees; here we simply need to add the orientation.

Let us begin with the following remark. Let $T$ and $T^{\prime}$ be two oriented $\mathbb{R}$-trees and $x_{1}, \ldots, x_{n} \in T, x_{1}^{\prime}, \ldots, x_{n}^{\prime} \in T^{\prime}$ such that for all $i, j, d\left(x_{i}, x_{j}\right)=d\left(x_{i}^{\prime}, x_{j}^{\prime}\right)$ and all $i, j, k, \operatorname{or}\left(x_{i}, x_{j}, x_{k}\right)=\operatorname{or}^{\prime}\left(x_{i}^{\prime}, x_{j}^{\prime}, x_{k}^{\prime}\right)$. Denote $K=\left\{x_{1}, \ldots, x_{n}\right\}$. Then the function $\varphi_{K}:\left\{x_{1}, \ldots, x_{n}\right\} \rightarrow\left\{x_{1}^{\prime}, \ldots, x_{n}^{\prime}\right\}$ defined by $\varphi_{K}\left(x_{i}\right)=x_{i}^{\prime}$ extends uniquely to an orientation-preserving isometry

$$
\varphi_{\operatorname{Hull}(K)}: \operatorname{Hull}\left(\left\{x_{1}, \ldots, x_{n}\right\}\right) \rightarrow \operatorname{Hull}\left(\left\{x_{1}^{\prime}, \ldots, x_{n}^{\prime}\right\}\right)
$$

(the subsets $\operatorname{Hull}\left(\left\{x_{1}, \ldots, x_{n}\right\}\right)$ and $\operatorname{Hull}\left(\left\{x_{1}^{\prime}, \ldots, x_{n}^{\prime}\right\}\right)$, as subtrees of $T$ and $T^{\prime}$, are oriented trees, by the restrictions of or and or $\left.^{\prime}\right)$. 
We prove this by induction. If $n=1$, there is not very much to do. Denote again $K=\left\{x_{1}, \ldots, x_{n}\right\}$ and suppose that $\varphi_{\mathrm{Hull}(K)}$ is an orientation-preserving isometry between $\operatorname{Hull}\left(\left\{x_{1}, \ldots, x_{n}\right\}\right)$ and $\operatorname{Hull}\left(\left\{x_{1}^{\prime}, \ldots, x_{n}^{\prime}\right\}\right)$. Denote by $y_{n+1}$ the projection of $x_{n+1}$ on $\operatorname{Hull}(K)$. The following relation, true in every $\mathbb{R}$-tree,

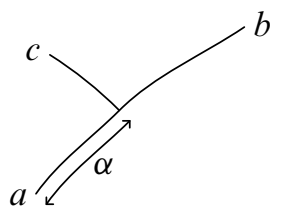

$$
\alpha=\frac{1}{2}(d(a, b)+d(a, c)-d(b, c)),
$$

allows us to find $y_{n+1}$ in the tree $\operatorname{Hull}(K)$ : the real numbers $d\left(x_{1}, x_{n+1}\right), \ldots$, $d\left(x_{n}, x_{n+1}\right)$ determine a unique point $y_{n+1} \in \operatorname{Hull}(K)$; similarly they determine a unique point $y_{n+1}^{\prime} \in \operatorname{Hull}\left(\left\{x_{1}^{\prime}, \ldots, x_{n}^{\prime}\right\}\right)$, and $\varphi_{\mathrm{Hull}(K)}\left(y_{n+1}\right)=y_{n+1}^{\prime}$. If $x_{n+1}=$ $y_{n+1}$ then $\operatorname{Hull}\left(\left\{x_{1}, \ldots, x_{n}\right\}\right)=\operatorname{Hull}\left(\left\{x_{1}, \ldots, x_{n+1}\right\}\right)$ and $\operatorname{Hull}\left(\left\{x_{1}^{\prime}, \ldots, x_{n}^{\prime}\right\}\right)=$ $\operatorname{Hull}\left(\left\{x_{1}^{\prime}, \ldots, x_{n+1}^{\prime}\right\}\right)$ and the induction is proved. Otherwise, Hull $\left(\left\{x_{1}, \ldots, x_{n+1}\right\}\right)$ is obtained by gluing at the point $y_{n+1}$ the tree $\operatorname{Hull}\left(\left\{x_{1}, \ldots, x_{n}\right\}\right)$ and the segment $\left[x_{n+1}, y_{n+1}\right]$, whose length is determined by the real numbers $d\left(x_{1}, x_{n+1}\right), \ldots$, $d\left(x_{n}, x_{n+1}\right)$; and similarly for $\operatorname{Hull}\left(\left\{x_{1}^{\prime}, \ldots, x_{n+1}^{\prime}\right\}\right)$ in $T^{\prime}$. In that way, the isometry $\varphi_{\mathrm{Hull}(K)}$ extends to a unique isometry $\varphi_{\mathrm{Hull}\left(\left\{x_{1}, \ldots, x_{n+1}\right\}\right)}$. Since $\varphi_{\mathrm{Hull}(K)}$ preserves the orientation, we need only check that $\varphi_{\operatorname{Hull}\left(\left\{x_{1}, \ldots, x_{n+1}\right\}\right)}$ preserves the orientation at the vertex $y_{n+1}$. But this follows from the fact that all the classes of the tripods of centre $y_{n+1}$ have a representant of the type $\operatorname{Hull}\left(\left\{x_{i}, x_{j}, x_{n+1}\right\}\right)$, where $\operatorname{Card}\{i, j, n+1\}=3$.

Now suppose that $(\rho, T)$ and $\left(\rho^{\prime}, T^{\prime}\right)$ define the same functions $f$ and $g$; denote by $x_{0}$ and $x_{0}^{\prime}$ the base points. Let $P$ be a finite subset of $\Gamma$. We then have a unique isometry $\varphi_{\mathrm{Hull}\left(P \cdot x_{0}\right)}$ between $\operatorname{Hull}\left(P \cdot x_{0}\right)$ and $\operatorname{Hull}\left(P \cdot x_{0}^{\prime}\right)$, preserving the orientation, such that $\varphi_{\text {Hull }\left(P \cdot x_{0}\right)}\left(\gamma \cdot x_{0}\right)=\gamma x_{0}^{\prime}$. In particular, for every finite subset $Q$ of $P$ the restriction of $\varphi_{\mathrm{Hull}\left(P \cdot x_{0}\right)}$ to $\operatorname{Hull}\left(Q \cdot x_{0}\right)$ equals $\varphi_{\mathrm{Hull}}\left(Q \cdot x_{0}\right)$, so that we can construct an isometry $\varphi: \bigcup_{P \subset \Gamma} \operatorname{Hull}\left(P \cdot x_{0}\right) \rightarrow \bigcup_{P \subset \Gamma} \operatorname{Hull}\left(P \cdot x_{0}^{\prime}\right)$, such that for all $\gamma \in \Gamma$ we have $\varphi\left(\gamma \cdot x_{0}\right)=\gamma \cdot x_{0}^{\prime}$; and we also deduce that for every $\gamma \in \Gamma$ and every finite subset $P$ of $\Gamma$ we have

$$
\forall y \in \operatorname{Hull}\left(P \cdot x_{0}\right), \quad \rho^{\prime}(\gamma) \cdot \varphi_{\operatorname{Hull}\left(P \cdot x_{0}\right)}=\varphi_{\operatorname{Hull}\left(\gamma P \cdot x_{0}\right)}(\rho(\gamma) \cdot y),
$$

which ensures that the isometry $\varphi$ is equivariant for the actions $\rho, \rho^{\prime}$ on the trees $\bigcup_{P \subset \Gamma} \operatorname{Hull}\left(P \cdot x_{0}\right)$ and $\bigcup_{P \subset \Gamma} \operatorname{Hull}\left(P \cdot x_{0}^{\prime}\right)$. Every tripod in $\bigcup_{P \subset \Gamma} \operatorname{Hull}\left(P \cdot x_{0}\right)$ is in $\operatorname{Hull}\left(P \cdot x_{0}\right)$ for $P$ big enough, hence $\varphi$ preserves the orientation. Finally, since the actions $\rho$ and $\rho^{\prime}$ are minimal, we have $\bigcup_{P \subset \Gamma} \operatorname{Hull}\left(P \cdot x_{0}\right)=T$ and $\bigcup_{P \subset \Gamma} \operatorname{Hull}\left(P \cdot x_{0}^{\prime}\right)=T^{\prime}$; whence $\varphi: T \rightarrow T^{\prime}$ is an orientation-preserving equivariant isometry such that $\varphi\left(x_{0}\right)=x_{0}^{\prime}$. 
Of course, $f$ and $g$ depend on $x_{0}$, anyway it follows from this proposition that the classes of minimal actions of $\Gamma$ on oriented $\mathbb{R}$-trees not reduced to a point, up to orientation-preserving equivariant isometry, form a set, which we denote by $\mathcal{T}^{\prime \prime}(\Gamma)$. We denote by $\mathcal{T}^{o}(\Gamma)$ its subset formed by those $(\rho, T) \in \mathcal{T}^{\prime \prime}(\Gamma)$ such that $\min _{x_{0} \in T} \max _{\gamma \in S} d_{T}\left(x_{0}, \gamma \cdot x_{0}\right)=1$ and such that whenever $\rho$ possesses at least one global fixed point in $\partial_{\infty} T$, the tree $T$ is isometric to $\mathbb{R}$.

Our aim now is to define a topology on the set $m_{\Gamma}^{o}(2) \cup \mathcal{T}^{o}(\Gamma)$.

3.2.2 Rigidity of the order We first need to give some technical lemmas indicating that the orders given by triples of points in oriented $\mathbb{R}$-trees, as well as in $\mathbb{H}^{2}$, are stable under small perturbations of the tree or of the plane. In all this section, $X$ will be an oriented $\mathbb{R}$-tree or the hyperbolic plane $\mathbb{H}^{2}$, equipped with its orientation (and hence, with a total cyclic order on its boundary), and with a metric $d_{\mathbb{H}^{2}} / d$ proportional to its usual metric. Its best constant of hyperbolicity is then $\delta(X)=\delta\left(\mathbb{H}^{2}\right) / d$.

Lemma 3.10 Let $x_{1}, x_{2}, x_{3} \in X$. Then there exists a unique $x_{0} \in X$ which minimises the function $x \mapsto d\left(x, x_{1}\right)+d\left(x, x_{2}\right)+d\left(x, x_{3}\right)$. Moreover, the function $X^{3} \rightarrow X$ defined by $\left(x_{1}, x_{2}, x_{3}\right) \mapsto x_{0}$ is continuous.

Proof If $X$ is an $\mathbb{R}$-tree, then we check easily that the unique point $m \in X$ such that $\left[x_{1}, x_{2}\right] \cap\left[x_{1}, x_{3}\right]=\left[x_{1}, m\right]$ is the point $x_{0}$ wanted. If $X$ is the hyperbolic plane $\mathbb{H}^{2}$ equipped with a metric proportional to $d_{\mathbb{H}^{2}}$, then the function $x \mapsto d\left(x, x_{1}\right)+$ $d\left(x, x_{2}\right)+d\left(x, x_{3}\right)$ is convex, proper, hence achieves a minimum. And the CAT( 0$)$ inequality implies that this function cannot be constant on any nondegenerate segment, hence this minimum is unique. Moreover, this convex function depends continuously on $x_{1}, x_{2}$ and $x_{3}$, hence its unique minimum also depends continuously on $x_{1}, x_{2}$ and $x_{3}$.

Remark 3.11 In the Euclidean plane $\mathbb{R}^{2}$, the point $x_{0}$ is called the Fermat point of the triangle $\Delta\left(x_{1}, x_{2}, x_{3}\right)$. If this triangle has angles smaller than $2 \pi / 3$, then this point coincides with the Torricelli point, which, in that case, sees every edge of the triangle under an angle equal to $2 \pi / 3$ (see eg [12]). When one of the angles of the triangle is at least $2 \pi / 3$, this Fermat point coincides with the corresponding vertex.

Let $A \geq 0$. We denote by $V(A) \subset X^{3}$ the set of $\left(x_{1}, x_{2}, x_{3}\right) \in X^{3}$ such that for every permutation $(i, j, k)$ of $(1,2,3)$, we have $d\left(x_{i}, x_{j}\right)+d\left(x_{j}, x_{k}\right)-d\left(x_{i}, x_{k}\right)>A$. Note that $V(A) \subset V\left(A^{\prime}\right)$ if $A^{\prime} \leq A$.

Lemma 3.12 For all $\left(x_{1}, x_{2}, x_{3}\right) \in V(6 \delta(X))$, we have $x_{0} \notin\left\{x_{1}, x_{2}, x_{3}\right\}$, where $x_{0}$ is as in Lemma 3.10. 
Proof Let $x_{1}, x_{2}, x_{3} \in X$ such that $\left(x_{1}, x_{2}, x_{3}\right) \in V(6 \delta(X))$. By definition of hyperbolicity, there exists a point $a \in X$ at distance at most $\delta(X)$ of each of the geodesic segments $\left[x_{i}, x_{j}\right]$. The triangle inequality then gives

$$
2 d\left(a, x_{1}\right)+2 d\left(a, x_{2}\right)+2 d\left(a, x_{3}\right) \leq 6 \delta(X)+d\left(x_{1}, x_{2}\right)+d\left(x_{2}, x_{3}\right)+d\left(x_{3}, x_{1}\right) .
$$

Since $\left(x_{1}, x_{2}, x_{3}\right) \in V(6 \delta(X))$, it follows that $d\left(a, x_{1}\right)+d\left(a, x_{2}\right)+d\left(a, x_{3}\right)<$ $d\left(x_{1}, x_{2}\right)+d\left(x_{1}, x_{3}\right)$, and similarly when permuting $x_{1}, x_{2}$ and $x_{3}$; and by definition of $x_{0}$ it follows that $x_{0} \notin\left\{x_{1}, x_{2}, x_{3}\right\}$.

From now on, suppose that $X$ is either the hyperbolic plane or an $\mathbb{R}$-tree with extendible segments. Define a set $U \subset X^{6}$ as follows: say that $\left(x_{1}, x_{2}, x_{3}, y_{1}, y_{2}, y_{3}\right) \notin U$ if there exist $i, j \in\{1,2,3\}, i \neq j$, and rays $r_{i}, r_{j}$ starting at $x_{i}, x_{j}$ and passing through $y_{i}, y_{j}$ respectively, such that $r_{i}, r_{j}$ represent the same point of $\partial_{\infty} X$, in the case when $X$ is a tree (heuristically, $\left(x_{1}, \ldots, y_{3}\right) \in U$ if the oriented segments $\left[x_{i}, y_{i}\right]$ point three distinct directions). This implies, in particular, that $x_{i} \neq y_{i}$, for all $i$. If $X=\mathbb{H}^{2}$, then there is a unique ray (up to parametrisation) issued from $x_{i}$ and passing through $y_{i}$; the condition $\left(x_{1}, \ldots, y_{3}\right) \in U$ expresses the fact that the ends of these three rays are three distinct points of $\partial \mathbb{H}^{2}$. In the case of an $\mathbb{R}$-tree, we can also give the following equivalent condition:

Lemma 3.13 Let $X$ be an $\mathbb{R}$-tree with extendible segments. Then $\left(x_{1}, \ldots, y_{3}\right) \in U$ if and only if $y_{1}, y_{2}$ and $y_{3}$ are pairwise distinct, and for every $i \in\{1,2,3\}$, the points $x_{i}, y_{i+1}$ and $y_{i+2}$ (we are using here a cyclic notation for the indices) are in the same connected component of $X \backslash\left\{y_{i}\right\}$.

Proof We first check that $\left(x_{1}, \ldots, y_{3}\right) \in U$ implies that $y_{1}, y_{2}$ and $y_{3}$ are not aligned. Suppose that $y_{1}, y_{2}$ and $y_{3}$ are three pairwise distinct points, and are aligned (the case when some of them coincide is treated similarly). For instance, take $y_{2} \in\left[y_{1}, y_{3}\right]$. If $\left[y_{1}, x_{1}\right]$ and $\left[y_{1}, y_{2}\right]$ define the same germ of oriented segments and if $\left[y_{3}, x_{3}\right]$ and $\left[y_{3}, y_{2}\right]$ also define the same germ of oriented segments, then, regardless of the position of $x_{2}$ there exists a ray starting at $x_{2}$, passing through $y_{2}$, and having the same end as some ray starting at $x_{i}$ and passing through $y_{i}$, for some $i \in\{1,3\}$, contradicting the definition of $U$. Suppose then, for instance, that the oriented segments $\left[y_{1}, x_{1}\right]$ and $\left[y_{1}, y_{2}\right]$ are in distinct germs. Then $\left[y_{3}, x_{3}\right]$ and $\left[y_{3}, y_{2}\right]$ have to be in distinct germs, otherwise there would exist two rays, one starting at $x_{3}$ and passing through $y_{3}$, the other starting at $x_{1}$, and passing through $y_{1}$ and then $y_{3}$, going to the same end. Now, it is again impossible to place $x_{2}$ in such a way that $\left(x_{1}, \ldots, y_{3}\right)$ is in $U$. Suppose now that $y_{1}, y_{2}, y_{3}$ are not aligned, and suppose, say, that the oriented segments $\left[y_{1}, x_{1}\right]$ and $\left[y_{1}, y_{2}\right]$ are in distinct germs (this is equivalent to the condition that $x_{1}$ and $y_{2}$ 
(or $y_{3}$ ) lie in two distinct components of $X \backslash\left\{y_{2}\right\}$ ). Then $x_{2}$ is impossible to position so that $\left(x_{1}, \ldots, y_{3}\right)$ can be in $U$ : indeed if $\left[y_{2}, x_{2}\right]$ and $\left[y_{2}, y_{1}\right]$ are in distinct germs then $\left[x_{1}, y_{3}\right]$ and $\left[x_{2}, y_{3}\right]$ pass through $y_{1}$ and $y_{2}$ respectively, and can be continued in the same way; otherwise $\left[x_{1}, y_{2}\right]$ and $\left[x_{2}, y_{2}\right]$ can be continued in the same way (and $\left[x_{1}, y_{2}\right]$ passes through $y_{1}$ ). The condition of the lemma is therefore necessary, and is obviously sufficient: for each $i$, the segment $\left[x_{i}, y_{i}\right]$ has to be continued (in order to form a ray) on a connected component of $X \backslash\left\{y_{i}\right\}$ different from the one where $y_{i+1}$ and $y_{i+2}$ lie.

In this section, $X$ is either the hyperbolic plane or an oriented $\mathbb{R}$-tree. In both cases, its boundary $\partial_{\infty} X$ is equipped with a total cyclic order $\mathbf{0}$.

Lemma 3.14 If $\left(x_{1}, \ldots, y_{3}\right) \in U$ and if $r_{i}, r_{i}^{\prime}$ are rays issued from $x_{i}$ and passing through $y_{i}$, then $\mathbf{o}\left(r_{1}, r_{2}, r_{3}\right)=\mathbf{o}\left(r_{1}^{\prime}, r_{2}^{\prime}, r_{3}^{\prime}\right)$.

We write $o\left(x_{1}, \ldots, y_{3}\right)=\mathbf{o}\left(r_{1}, r_{2}, r_{3}\right)$ in that case.

Proof We have $x_{i} \neq y_{i}$ so in the case when $X=\mathbb{H}^{2}$, there exists a unique ray $r_{i}$ issued from $x_{i}$ and passing through $y_{i}$. Now suppose that $X$ is an oriented $\mathbb{R}-$ tree. By Lemma 3.13, $y_{1}, y_{2}$ and $y_{3}$ are not aligned; let then $y_{0}$ be such that $\left[y_{1}, y_{2}\right] \cap\left[y_{1}, y_{3}\right]=\left[y_{1}, y_{0}\right]$. Then $y_{1}, y_{2}, y_{3}$ define three distinct germs of rays issued from $y_{0}$. Still by Lemma 3.13, the condition $\left(x_{1}, \ldots, y_{3}\right) \in U$ implies that for every $i$, $x_{i}$ lies in the connected component of $X \backslash\left\{y_{i}\right\}$ containing $y_{0}$. In particular, every ray $r$ issued from $x_{i}$ and passing through $y_{i}$ defines a unique ray issued from $y_{0}$ and passing through $y_{i}$ : it is the ray joining $y_{0}$ to $y_{i}$, and which then continues as the ray $r$. Therefore, the equality $\mathbf{o}\left(r_{1}, r_{2}, r_{3}\right)=\mathbf{o}\left(r_{1}^{\prime}, r_{2}^{\prime}, r_{3}^{\prime}\right)$ follows from the coherence condition on the order $\mathbf{0}$ of the oriented $\mathbb{R}$-tree we are considering.

Lemma 3.15 The function $o: U \rightarrow\{-1,1\}$ thereby defined is continuous.

Proof If $X=\mathbb{H}^{2}$, it is immediate that the function $X^{2} \backslash \Delta \rightarrow \partial X$ (where $\Delta$ is the diagonal) sending $(x, y)$ on the end of the ray issued from $x$ and passing through $y$ is continuous (where $\partial X=\mathbb{S}^{1}$ is equipped with the usual topology), and hence $o: U \rightarrow\{-1,1\}$ is simply the composition of two continuous functions. In the case when $X$ is an oriented $\mathbb{R}$-tree, the proof is similar to that of the preceding lemma.

Lemma 3.16 Let $x_{1}, \ldots, y_{3}$ such that for every $i \in\{1,2,3\}$, we have $\sum_{j} d\left(x_{i}, y_{j}\right)<$ $\sum_{j} d\left(y_{i}, y_{j}\right)$. Then $\left(x_{1}, \ldots, y_{3}\right) \in U$. 
Proof Let $x_{1}, \ldots, y_{3} \in X$ with $\left(x_{1}, \ldots, y_{3}\right) \notin U$. Then, up to changing the indices, there exist rays $r_{1}$ and $r_{2}$ extending the oriented segments $\left[x_{1}, y_{1}\right]$ and $\left[x_{2}, y_{2}\right]$, with $\left[r_{1}\right]=\left[r_{2}\right]$ in $\partial_{\infty} X$. Up to changing the indices again, we may suppose that $y_{2}$ is "closer" to this point at infinity than $y_{1}$, in the sense that

$$
\lim _{t \rightarrow+\infty}\left(d\left(y_{1}, r_{1}(t)\right)-d\left(y_{2}, r_{1}(t)\right)\right) \geq 0 .
$$

We will prove that $d\left(x_{1}, y_{2}\right) \geq d\left(y_{1}, y_{2}\right)$. With the triangle inequality, this will imply that $d\left(x_{1}, y_{1}\right)+d\left(x_{1}, y_{2}\right)+d\left(x_{1}, y_{3}\right) \geq d\left(y_{1}, y_{2}\right)+d\left(y_{1}, y_{3}\right)$, hence the lemma.

The limit (3-3) gives a Euclidean comparison triangle

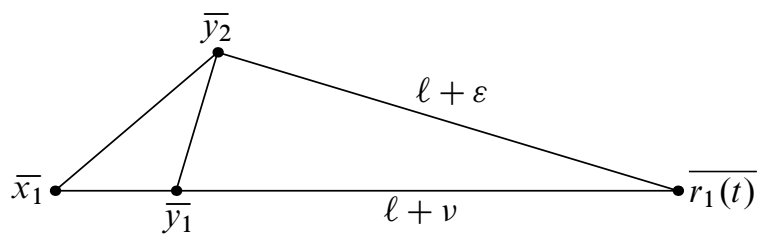

with $v \geq 0$ fixed, $\varepsilon$ as small as we want and $\ell$ as large as we want, for $t$ big enough. The CAT(0) inequality then actually implies $d\left(x_{1}, y_{2}\right)>d\left(y_{1}, y_{2}\right)$.

In particular, if $\left(x_{1}, x_{2}, x_{3}\right) \in V(6 \delta(X))$ and if $x_{0}$ realizes the minimum of the function $x \mapsto d\left(x, x_{1}\right)+d\left(x, x_{2}\right)+d\left(x, x_{3}\right)$, then we have $\left(x_{0}, x_{0}, x_{0}, x_{1}, x_{2}, x_{3}\right) \in U$. We set $o\left(x_{1}, x_{2}, x_{3}\right)=o\left(x_{0}, \ldots, x_{3}\right)$ in that case.

Remark 3.17 Let $y_{1}, y_{2}, y_{3} \in X$. The hypotheses of Lemma 3.16 being convex conditions on $x_{1}, x_{2}$ and $x_{3}$, they define convex subsets of $X$. In particular, by Lemma 3.15, if $\left(y_{1}, y_{2}, y_{3}\right) \in V(6 \delta(X))$ and if for every $i \in\{1,2,3\}, \sum_{j} d\left(x_{i}, y_{j}\right)<$ $\sum_{j} d\left(y_{i}, y_{j}\right)$, then we have $o\left(x_{1}, \ldots, y_{3}\right)=o\left(y_{1}, y_{2}, y_{3}\right)$.

Remark 3.18 Suppose that the spaces $X$ and $X^{\prime}$ are the hyperbolic plane (equipped with a distance proportional to the usual distance) or an $\mathbb{R}$-tree, and let $x_{1}, x_{2}, x_{3} \in X$, $x_{1}^{\prime}, x_{2}^{\prime}, x_{3}^{\prime} \in X^{\prime}$. Suppose that $\left(x_{1}, x_{2}, x_{3}\right) \in V(6 \delta(X)+a),\left|\delta(X)-\delta\left(X^{\prime}\right)\right|<\varepsilon_{1}$, and $\left|d\left(x_{i}, x_{j}\right)-d\left(x_{i}^{\prime}, x_{j}^{\prime}\right)\right|<\varepsilon_{2}$ for all $i, j \in\{1,2,3\}$. Then we have $\left(x_{1}^{\prime}, x_{2}^{\prime}, x_{3}^{\prime}\right) \in$ $V\left(6 \delta\left(X^{\prime}\right)+a-6 \varepsilon_{1}-3 \varepsilon_{2}\right)$.

Therefore, the sets $V(A)$ provide open conditions, robust under $\varepsilon$-approximations (for $\varepsilon$ sufficiently small, given $A$ ), guaranteeing that we can consider the orientations defined by triples of points. 
Notation In all this text, regardless whether $X$ is an oriented $\mathbb{R}$-tree or the hyperbolic plane, we use (coherent) cyclic orders on $\partial_{\infty} X$, which are always denoted by a bold letter (o). This is necessary for our treatment of the Euler class. In order to compare spaces and use an equivariant Gromov topology, we also need to use a local version of this same information: if $X$ is an oriented $\mathbb{R}$-tree, its orientation (which is a function on $\operatorname{Trip}(X))$ is always denoted by or. And in both cases, if three points are sufficiently far from being aligned (this is the $V(6 \delta)$ ) condition) they come in a certain order, which will always denoted by a simple letter $o$. In what follows, an element of $m_{\Gamma}^{o}(2) \cup \mathcal{T}^{o}(\Gamma)$, be it an action on $\mathbb{H}^{2}$ or on a tree, will be denoted by $(\rho, X, d, o)$, where $o$ is the function $V(6 \delta(X)) \rightarrow\{-1,1\}$ we have just defined.

\subsubsection{Oriented equivariant Gromov topology}

Definition 3.19 Let $(\rho, X, d, o)$ and $\left(\rho^{\prime}, X^{\prime}, d^{\prime}, o^{\prime}\right) \in m_{\Gamma}^{o}(2) \cup \mathcal{T}^{o}(\Gamma)$. Let $K=$ $\left(x_{1}, \ldots, x_{p}\right)$ and $K^{\prime}=\left(x_{1}^{\prime}, \ldots, x_{p}^{\prime}\right)$ be finite sequences in $X$ and $X^{\prime}$, respectively. Let $\varepsilon>0$ and let $P$ be a finite subset of $\Gamma$, with $1 \in P$. We say that $K^{\prime}$ is an oriented $P$-equivariant $\varepsilon$-approximation of $K$ if it is a $P$-equivariant $\varepsilon$-approximation of $K$ and if, moreover, for all $\left(x_{i}, x_{j}, x_{k}\right) \in V(6 \delta(X)+9 \varepsilon)$, we have $o\left(x_{i}, x_{j}, x_{k}\right)=$ $o^{\prime}\left(x_{i}^{\prime}, x_{j}^{\prime}, x_{k}^{\prime}\right)$.

If $\left(x_{i}, x_{j}, x_{k}\right) \in V(6 \delta(X)+9 \varepsilon)$, it follows from Remark 3.18 that both $o\left(x_{i}, x_{j}, x_{k}\right)$ and $o^{\prime}\left(x_{i}^{\prime}, x_{j}^{\prime}, x_{k}^{\prime}\right)$ are well defined, hence Definition 3.19 makes sense.

Now if $(\rho, X) \in m_{\Gamma}^{o}(2) \cup \mathcal{T}^{o}(\Gamma)$, if $\varepsilon>0$, if $K=\left(x_{1}, \ldots, x_{p}\right)$ is a finite sequence in $X$ and if $P$ is a finite subset of $\Gamma$ containing 1 , we denote by $U_{K, \varepsilon, P}^{\prime \prime}(\rho, X)$ the set of $\left(\rho^{\prime}, X^{\prime}\right) \in m_{\Gamma}^{o}(2) \cup \mathcal{T}^{o}(\Gamma)$ such that $X^{\prime}$ contains an oriented $P$-equivariant $\varepsilon$-approximation of $K$.

Proposition 3.20 The sets $U_{K, \varepsilon, P}^{\prime \prime}(\rho, X)$ form the basis of open sets of some topology; we call it the oriented equivariant Gromov topology.

Proof Of course, we always have $(\rho, X) \in U_{K, \varepsilon, P}^{\prime \prime}(\rho, X)$. Hence, we need only check that if $(\rho, X) \in U_{K_{1}, \varepsilon_{1}, P_{1}}^{\prime \prime}\left(\rho_{1}, X_{1}\right) \cap U_{K_{2}, \varepsilon_{2}, P_{2}}^{\prime \prime}\left(\rho_{2}, X_{2}\right)$ then, for some $K, \mu>0$ and $P$ such that $1 \in P$, we have $U_{K, \mu, P}^{\prime \prime}(\rho, X) \subset U_{K_{1}, \varepsilon_{1}, P_{1}}^{\prime \prime}\left(\rho_{1}, X_{1}\right) \cap U_{K_{2}, \varepsilon_{2}, P_{2}}^{\prime \prime}\left(\rho_{2}, X_{2}\right)$. It is just a technical verification. Denote $K_{1}=\left(a_{1}^{\prime}, \ldots, a_{n_{1}}^{\prime}\right), K_{2}=\left(a_{n_{1}+1}^{\prime}, \ldots, a_{n_{1}+n_{2}}^{\prime}\right)$. Then $\left|\delta(X)-\delta\left(X_{1}\right)\right|<\varepsilon_{1},\left|\delta(X)-\delta\left(X_{2}\right)\right|<\varepsilon_{2}$, and there exists a finite sequence $K=\left(a_{1}, \ldots, a_{n_{1}+n_{2}}\right) \subset X$ such that

$\forall i, j \leq n_{1}, \forall \gamma_{1}, \gamma_{2} \in P_{1},\left|d_{X}\left(\rho\left(\gamma_{1}\right) \cdot a_{i}, \rho\left(\gamma_{2}\right) \cdot a_{j}\right)-d_{X_{1}}\left(\rho_{1}\left(\gamma_{1}\right) \cdot a_{i}^{\prime}, \rho_{1}\left(\gamma_{2}\right) \cdot a_{j}^{\prime}\right)\right|<\varepsilon_{1}$, 
and such that $o_{X_{1}}\left(a_{i}^{\prime}, a_{j}^{\prime}, a_{k}^{\prime}\right)=o_{X}\left(a_{i}, a_{j}, a_{k}\right)$ for every $i, j, k \leq n_{1}$ that satisfies $\left(a_{i}^{\prime}, a_{j}^{\prime}, a_{k}^{\prime}\right) \in V\left(6 \delta\left(X_{1}\right)+9 \varepsilon_{1}\right)$, and similarly for elements $a_{i}, a_{j}$ and $a_{k}$ with indices $i, j, k \geq n_{1}+1$. Since all these finitely many inequalities are strict, there exists $\mu>0$ such that

$$
\begin{gathered}
\left|d_{X}\left(\rho\left(\gamma_{1}\right) \cdot a_{i}, \rho\left(\gamma_{2}\right) \cdot a_{j}\right)-d_{X_{1}}\left(\rho_{1}\left(\gamma_{1}\right) \cdot a_{i}^{\prime}, \rho_{1}\left(\gamma_{2}\right) \cdot a_{j}^{\prime}\right)\right|<\varepsilon_{1}-\mu, \\
\left|\delta(X)-\delta\left(X_{1}\right)\right|<\varepsilon_{1}-\mu,
\end{gathered}
$$

and such for all $\left(a_{i}^{\prime}, a_{j}^{\prime}, a_{k}^{\prime}\right) \in V\left(6 \delta\left(X_{1}\right)+9 \varepsilon_{1}\right)$ we actually have

$$
\left(a_{i}^{\prime}, a_{j}^{\prime}, a_{k}^{\prime}\right) \in V\left(6 \delta\left(X_{1}\right)+9 \varepsilon_{1}+9 \mu\right),
$$

for all $i, j, k \leq n_{1}$, and such that the similar inequalities hold, concerning $X_{2}$.

Now, take $\left(\rho^{\prime \prime}, X^{\prime \prime}\right) \in U_{K, \mu, P_{1} \cup P_{2}}^{\prime \prime}(\rho, X)$, and let $K^{\prime \prime}=\left(a_{1}^{\prime \prime}, \ldots, a_{n_{1}+n_{2}}^{\prime \prime}\right) \subset X^{\prime \prime}$ be an oriented $P_{1} \cup P_{2}$-equivariant $\mu$-approximation of $K$. Then in particular, for every $i, j \leq n_{1}$ and every $\gamma_{1}, \gamma_{2} \in P_{1}$,

$$
\left|d_{X^{\prime \prime}}\left(\rho^{\prime \prime}\left(\gamma_{1}\right) \cdot a_{i}^{\prime \prime}, \rho^{\prime \prime}\left(\gamma_{2}\right) \cdot a_{j}^{\prime \prime}\right)-d_{X}\left(\rho\left(\gamma_{1}\right) \cdot a_{i}, \rho\left(\gamma_{2}\right) \cdot a_{j}\right)\right|<\mu,
$$

$\left|\delta(X)-\delta\left(X^{\prime \prime}\right)\right|<\mu$, and for every $i, j, k$ such that $\left(a_{i}, a_{j}, a_{k}\right) \in V(6 \delta(X)+9 \mu)$, $o_{X}\left(a_{i}, a_{j}, a_{k}\right)=o_{X^{\prime \prime}}\left(a_{i}^{\prime \prime}, a_{j}^{\prime \prime}, a_{k}^{\prime \prime}\right)$. Now, the conditions (3-4) and (3-5) imply that for every $i, j \leq n_{1}$ and every $\gamma_{1}, \gamma_{2} \in P_{1}$,

$$
\left|d_{X^{\prime \prime}}\left(\rho^{\prime \prime}\left(\gamma_{1}\right) \cdot a_{i}^{\prime \prime}, \rho^{\prime \prime}\left(\gamma_{2}\right) \cdot a_{j}^{\prime \prime}\right)-d_{X_{1}}\left(\rho_{1}\left(\gamma_{1}\right) \cdot a_{i}^{\prime}, \rho_{1}\left(\gamma_{2}\right) \cdot a_{j}^{\prime}\right)\right|<\varepsilon_{1} .
$$

And for all $i, j, k \leq n_{1}$ such that $\left(a_{i}^{\prime}, a_{j}^{\prime}, a_{k}^{\prime}\right) \in V\left(6 \delta\left(X_{1}\right)+9 \varepsilon_{1}\right)$, we also have $\left(a_{i}^{\prime}, a_{j}^{\prime}, a_{k}^{\prime}\right) \in V\left(6 \delta\left(X_{1}\right)+9 \varepsilon_{1}+9 \mu\right)$, so $\left(a_{i}, a_{j}, a_{k}\right) \in V(6 \delta(X)+9 \mu)$, by Remark 3.18. Hence $o^{\prime \prime}\left(a_{i}^{\prime \prime}, a_{j}^{\prime \prime}, a_{k}^{\prime \prime}\right)=o^{\prime}\left(a_{i}^{\prime}, a_{j}^{\prime}, a_{k}^{\prime}\right)$, so finally $\left(\rho^{\prime \prime}, X^{\prime \prime}\right) \in U_{K_{1}, \varepsilon_{1}, P_{1}}^{\prime \prime}\left(\rho_{1}, X_{1}\right)$, and, similarly, $\left(\rho^{\prime \prime}, X^{\prime \prime}\right) \in U_{K_{2}, \varepsilon_{2}, P_{2}}^{\prime \prime}\left(\rho_{2}, X_{2}\right)$.

Proposition 3.21 The oriented equivariant Gromov topology coincides with the usual topology on $m_{\Gamma}^{o}(2)$.

Proof It is the same proof as the one of F Paulin's Proposition 6.2 in [35], with minor modifications. In that proof (recalled here, as Proposition 2.16), the only difference is that the isometry $\phi$ of Fact 2.17, is now an orientation-preserving isometry.

Of course, we denote by $\overline{m_{\Gamma}^{o}(2)}$ the closure of $m_{\Gamma}^{o}(2)$ in the space $m_{\Gamma}^{o}(2) \cup \mathcal{T}^{o}(\Gamma)$, equipped with the oriented equivariant Gromov topology.

We also write $\overline{m_{\Gamma}^{u}}=\overline{m_{\Gamma}^{u}(2)}$ and $\overline{m_{\Gamma}^{o}}=\overline{m_{\Gamma}^{o}(2)}$. 
3.2.4 The space $\overline{m_{\Gamma}^{o}}$ is compact Denote by $\pi: \overline{m_{\Gamma}^{o}} \rightarrow \overline{m_{\Gamma}^{u}}$ the natural function consisting in forgetting the orientation.

Proposition 3.22 The map $\pi$ is continuous, and its fibres are compact Hausdorff spaces.

Proof First, the continuity of $\pi$ follows directly from the definition of these two topologies.

Now, let $(\rho, T) \in \overline{m_{\Gamma}^{u}}$. If $T$ is $\mathbb{H}^{2}$, then the fibre $\pi^{-1}(\rho, T)$ has cardinal 2 in the Hausdorff space $m_{\Gamma}^{o}$ (by Theorem 2.5), hence it is compact Hausdorff. Suppose now that $T$ is an $\mathbb{R}$-tree. By definition, the set $\pi^{-1}(\rho, T)$ is a subset of $\operatorname{Or}(T)$. The induced topology on $\pi^{-1}(\rho, T)$ in $\operatorname{Or}(T)$ coincides with the oriented equivariant Gromov topology. Indeed, an open basis of the topology on $\operatorname{Or}(T)$ is given by the condition that some fixed germ of tripods $\operatorname{Trip}\left(a_{0}, a_{1}, a_{2}, a_{3}\right)$ is oriented positively, and by taking $K=\left\{a_{1}, a_{2}, a_{3}\right\}, \varepsilon$ small enough and $P=\{\mathrm{Id}\}$, this is open in the induced topology on $\overline{m_{\Gamma}^{o}}$. Reciprocally, in order to be in $U_{K, \varepsilon, P}^{\prime \prime}(\rho, T)$, a space $\left(\rho^{\prime}, X^{\prime}, d^{\prime}, o^{\prime}\right) \in \overline{m_{\Gamma}^{o}}$ needs to contain an oriented $P$-equivariant $\varepsilon$-approximation of $K$. For a given $\varepsilon-$ approximation $K^{\prime}$ of $K$, this amounts to check finitely many equalities, hence this is an open condition: thus, the intersection $\pi^{-1}(\rho, T) \cap U_{K, \varepsilon, P}^{\prime \prime}(\rho, T)$ is a union of open sets of $\operatorname{Or}(T)$.

We shall notice now that $\overline{m_{\Gamma}^{o}}$ is Hausdorff. Indeed, if $(\rho, X, d, o)$ and $\left(\rho^{\prime}, X^{\prime}, d^{\prime}, o^{\prime}\right)$ are distinct and are not separated by open sets, then $\delta(X)=\delta\left(X^{\prime}\right)$. The open set $m_{\Gamma}^{o}$ being Hausdorff, this means that $X$ and $X^{\prime}$ are oriented $\mathbb{R}$-trees. Since the map $\pi: \overline{m_{\Gamma}^{o}} \rightarrow \overline{m_{\Gamma}^{u}}$ is continuous, this implies that these two spaces differ only by the orientation: but by definition of the oriented equivariant Gromov topology, there are two open sets separating $(\rho, X, d, o)$ and $\left(\rho^{\prime}, X^{\prime}, d^{\prime}, o^{\prime}\right)$.

Finally, $\pi^{-1}(\rho, T)$ is the preimage of the point $(\rho, T)$ in the Hausdorff space $\overline{m_{g}^{u}}$ by the continuous map $\pi$, hence it is closed for the topology of $\overline{m_{g}^{o}}$, whose induced topology on $\pi^{-1}(\rho, T)$ coincides with that of $\operatorname{Or}(T)$, which is compact. Hence the fibre $\pi^{-1}(\rho, T)$ is compact.

Theorem 3.23 The space $\overline{m_{\Gamma}^{o}}$, equipped with the function $m_{\Gamma}^{o} \hookrightarrow \overline{m_{\Gamma}^{o}}$, is a natural compactification of $m_{\Gamma}^{o}$.

Here again, after [37], by "natural", we mean that the action of $\operatorname{Out}(\Gamma)$ on $m_{\Gamma}^{o}$ extends continuously to an action of $\operatorname{Out}(\Gamma)$ on $\overline{m_{\Gamma}^{o}}$. 
Proof Since $m_{\Gamma}^{o}$ is open and dense in $\overline{m_{\Gamma}^{o}}$ and since, by definition of the oriented equivariant Gromov topology, the action of $\operatorname{Out}(\Gamma)$ on $\overline{m_{\Gamma}^{o}}$ is continuous, it suffices to prove that the space $\overline{m_{\Gamma}^{o}}$ is compact Hausdorff. We have already seen that $\overline{m_{\Gamma}^{o}}$ is Hausdorff, and by the definition of compactness in terms of ultrafilters (see eg [5, page 59]), we need only prove that every ultrafilter in $\overline{m_{\Gamma}^{o}}$ converges.

Let $\omega$ be an ultrafilter in $\overline{m_{\Gamma}^{o}}$. Then the image of the ultrafilter $\pi(\omega)$ is an ultrafilter in the compact space $\overline{m_{\Gamma}^{u}}$ (see eg [5, Proposition 10, page 41]), hence it converges to some action $\left(\rho_{\infty}, X_{\infty}\right) \in \overline{m_{\Gamma}^{u}}$. If $X_{\infty}=\mathbb{H}^{2}$, then it follows from Proposition 3.21 that $X$ is equipped with an orientation, compatible with the convergence of the ultrafilter. We need only prove that if $X_{\infty}$ is an $\mathbb{R}$-tree (denote $(\rho, T)=\left(\rho_{\infty}, X_{\infty}\right)$ in that case) then there exists an orientation or $\in \operatorname{Or}(T)$, which is invariant under the action of $\Gamma$, and such that $(\rho, T)$, equipped with this order, is indeed the limit, in $\overline{m_{\Gamma}^{o}}$, of the ultrafilter $\omega$.

Consider an increasing sequence $T_{k} \subset T$ of finite, closed subtrees of $T$, such that $\bigcup_{k \geq 1} T_{k}=T$. Suppose for simplicity that $T_{1}$ is a singleton $\left\{x_{0}\right\}$. For all $k \geq 1$, denote by $F_{k}$ the finite sequence (let us simply pick these finitely many points in some arbitrary order) of all elements of $T_{k}$ whose distance to $x_{0}$ is a multiple of $1 / 2^{k}$, as well as all the end points and all the vertices of $T_{k}$, and denote by $\mu_{k}$ the smallest distance between two points in $F_{k}$. Since the ultrafilter $\pi(\omega)$ converges to $(\rho, T)$, for all $k \geq 1, \varepsilon>0$, and for every finite subset $P \subset \Gamma$ containing 1 , for all $M \in \omega$, there exists $\left(\rho_{M}, X_{M}, d_{M}, o_{M}\right) \in M$ such that $\left(\rho_{M}, X_{M}, d_{M}\right) \in$ $U_{F_{k}, \varepsilon, P}^{\prime}(\rho, T)$, ie there exists a $P$-equivariant $\varepsilon$-approximation between $F_{k}$ and some finite sequence $K_{M}$ in $X_{M}$. For all $M \in \omega$, denote by $O_{k, \varepsilon, P, M}$ the set of functions preor $\in \operatorname{Preor}(T)$ such that there exists such an approximation, such that for every $x_{1}, x_{2}, x_{3} \in F_{k}$ with $\left(x_{1}, x_{2}, x_{3}\right) \in V(9 \varepsilon)$, and for all corresponding $x_{1}^{\prime}, x_{2}^{\prime}, x_{3}^{\prime} \in K_{M}$, we have preor(Trip $\left.\left(x_{1}, x_{2}, x_{3}\right)\right)=o_{M}\left(x_{1}^{\prime}, x_{2}^{\prime}, x_{3}^{\prime}\right)$ (this is indeed well defined, thanks to Remark 3.17).

We cut the end of the proof into the two following lemmas:

Lemma 3.24 For every $k, \varepsilon, P \subset \Gamma$ containing 1 and every $M \in \omega$, the set $O_{k, \varepsilon, P, M}$ is closed, and nonempty. Moreover, if $k>k^{\prime}, \varepsilon<\varepsilon^{\prime}, P^{\prime} \subset P$ and $M \subset M^{\prime}$, we have $O_{k, \varepsilon, P, M} \subset O_{k^{\prime}, \varepsilon^{\prime}, P^{\prime}, M^{\prime}}$.

By compactness of $\operatorname{Preor}(T)$, it follows that $\bigcap_{k, \varepsilon, P, M} O_{k, \varepsilon, P, M} \neq \varnothing$.

Lemma 3.25 Let preor $\in \bigcap_{k, \varepsilon, P, M} O_{k, \varepsilon, P, M}$. Then preor satisfies the conditions (3-1) and (3-2): it is an orientation. Moreover, it is invariant under the action of $\Gamma$, and the element $(\rho, T)$, equipped with the orientation preor, is the limit, in $\overline{m_{\Gamma}^{o}}$, of the ultrafilter $\omega$. 
Proof of Lemma 3.24 It follows from the definition that $O_{k, \varepsilon, P, M} \subset O_{k^{\prime}, \varepsilon^{\prime}, P^{\prime}, M^{\prime}}$ if $k>k^{\prime}, \varepsilon<\varepsilon^{\prime}, M \subset M^{\prime}$ and $P^{\prime} \subset P$ (indeed, we then have $F_{k^{\prime}} \subset F_{k}$ ). The hypotheses concern only $\operatorname{Trip}\left(T_{k}\right)$, which is a finite subset of $\operatorname{Trip}(T)$, and hence $O_{k, \varepsilon, P, M}$ is closed. We need to prove that it is also nonempty.

Let $\varepsilon>0$ be sufficiently small (see below) and let us choose a (nonoriented) $\varepsilon-$ approximation between $F_{k}$ and a finite sequence $K_{M} \subset X_{M}$, for some $X_{M}$ as above (such an approximation exists, since $\pi(\omega)$ converges to $(\rho, T)$ in $\left.\overline{m_{g}^{u}}\right)$. For every tripod $\operatorname{Trip}\left(a_{0}, a_{1}, a_{2}, a_{3}\right)$ with $a_{i} \in F_{k}$, put $\operatorname{preor}\left(\operatorname{Trip}\left(a_{0}, a_{1}, a_{2}, a_{3}\right)\right)=o_{X_{M}}\left(a_{1}^{\prime}, a_{2}^{\prime}, a_{3}^{\prime}\right)$ : all we need is to check that this is well defined. Hence, we need to check that whenever $\operatorname{Trip}\left(a_{0}, a_{1}, a_{2}, a_{3}\right)$ and $\operatorname{Trip}\left(a_{0}, b_{1}, b_{2}, b_{3}\right)$ define the same germ of tripods in $T_{k}$, the corresponding elements $a_{i}^{\prime}, b_{i}^{\prime}$ in $X_{M}$ satisfy $o_{M}\left(a_{1}^{\prime}, a_{2}^{\prime}, a_{3}^{\prime}\right)=o_{M}\left(b_{1}^{\prime}, b_{2}^{\prime}, b_{3}^{\prime}\right)$.

We can go from the triple $\left(a_{1}, a_{2}, a_{3}\right)$ to the triple $\left(b_{1}, b_{2}, b_{3}\right)$ by a sequence of moves consisting of replacing $a_{1}, a_{2}$ or $a_{3}$ by one of its close neighbours in $T_{k}$, hence we may suppose that $\left(b_{1}, b_{2}\right)=\left(a_{1}, a_{2}\right)$, and that $a_{3} \in\left[a_{0}, b_{3}\right]$. Denote $\ell_{1}=d_{T}\left(a_{0}, a_{1}\right)$, $\ell_{2}=d_{T}\left(a_{0}, a_{2}\right), \ell_{3}=d_{T}\left(a_{0}, a_{3}\right)$ and $\ell_{4}=d_{T}\left(a_{3}, b_{3}\right)$. All these lengths are greater or equal to $\mu_{k}$. Let $a^{\prime}(t), t \in[0,1]$ be the geodesic segment joining $a^{\prime}(0)=a_{3}^{\prime}$ and $a^{\prime}(1)=b_{3}^{\prime}$ in $X_{M}$. Then, denoting by $d$ the distance in $X_{M}$, we have

$$
\ell_{1}+\ell_{2}-\varepsilon \leq d\left(a_{1}^{\prime}, a_{2}^{\prime}\right) \leq \ell_{1}+\ell_{2}+\varepsilon,
$$

and $d\left(a_{1}^{\prime}, a^{\prime}(t)\right) \leq d\left(a_{1}^{\prime}, a_{3}^{\prime}\right)+d\left(a_{3}^{\prime}, a^{\prime}(t)\right) \leq \ell_{1}+\ell_{3}+\varepsilon+\left(\ell_{4}+\varepsilon\right) t$ hence $d\left(a_{1}^{\prime}, a^{\prime}(t)\right) \leq$ $\ell_{1}+\ell_{3}+t \ell_{4}+2 \varepsilon$, and similarly $d\left(a_{1}^{\prime}, a^{\prime}(t)\right) \geq d\left(a_{1}^{\prime}, b_{3}^{\prime}\right)-d\left(b_{3}^{\prime}, a^{\prime}(t)\right) \geq \ell_{1}+\ell_{3}+$ $\ell_{4}-\left(\ell_{4}-\varepsilon\right)(1-t)$ so

$$
\ell_{1}+\ell_{3}+t \ell_{4}-2 \varepsilon \leq d\left(a_{1}, a^{\prime}(t)\right) \leq \ell_{1}+\ell_{3}+t \ell_{4}+2 \varepsilon .
$$

Similarly, $\quad \ell_{2}+\ell_{3}+t \ell_{4}-2 \varepsilon \leq d\left(a_{2}, a^{\prime}(t)\right) \leq \ell_{2}+\ell_{3}+t \ell_{4}+2 \varepsilon$.

Together with the inequality $\delta\left(X_{M}\right) \leq \varepsilon$, all these inequalities imply $\left(a_{1}^{\prime}, a_{2}^{\prime}, a^{\prime}(t)\right) \in$ $V\left(6 \delta\left(X_{M}\right)\right)$ for all $t \in[0,1]$, provided that $11 \varepsilon \leq 2 \mu_{k}$. It then follows from the continuity of the order (and more precisely, from Lemma 3.10 and Lemma 3.15) that $o_{X_{M}}\left(a_{1}^{\prime}, a_{2}^{\prime}, a_{3}^{\prime}\right)=o_{X_{M}}\left(b_{1}^{\prime}, b_{2}^{\prime}, b_{3}^{\prime}\right)$.

Proof of Lemma 3.25 It is immediate that preor satisfies condition (3-1). Condition (3-2), as well as the invariance of preor under the action of $\Gamma$, are proved by considering a big enough subtree $T_{k}$ of $T$ containing the desired branched points, and by deriving the properties of preor from the corresponding properties for $o_{M}$, which are supposed to be true since $\left(\rho_{M}, X_{M}, d_{M}, o_{M}\right) \in \overline{m_{\Gamma}^{o}}$. As an example we prove that preor satisfies condition (3-2); the proof of its invariance under $\Gamma$ is similar. Let $a_{0}, \ldots, a_{4} \in T$ be such that $\operatorname{preor}\left(\operatorname{Trip}\left(a_{0}, a_{1}, a_{2}, a_{3}\right)\right)=\operatorname{preor}\left(\operatorname{Trip}\left(a_{0}, a_{1}, a_{3}, a_{4}\right)=1\right.$. Since we are considering germs of tripods, we may suppose that all these points 
$a_{0}, \ldots, a_{4}$ are in $T_{k}$, for some $k$. Then, for all $\varepsilon$-approximation between $F_{k}$ and $K_{M} \subset X_{M}$, with $\varepsilon$ small enough, we have $\left(a_{0}^{\prime}, a_{0}^{\prime}, a_{0}^{\prime}, a_{i}^{\prime}, a_{j}^{\prime}, a_{k}^{\prime}\right) \in U$ in $X_{M}$, and then the equality preor(Trip $\left(a_{0}, a_{1}, a_{2}, a_{4}\right)=1$ indeed follows from the fact that $o_{M}$ is defined (see Lemma 3.14 and the following line) by a cyclic order on $\partial_{\infty} X_{M}$, satisfying the third condition of Definition 2.20.

Now, for all $M \in \omega$, we have $U_{F_{k}, \varepsilon, P}^{\prime \prime}(\rho, T, o) \cap M \neq \varnothing$, and by density of $\bigcup_{k} F_{k}$ in $T$, we have $U_{K, \varepsilon, P}^{\prime \prime}(\rho, T, o) \cap M \neq \varnothing$ for every finite sequence $K$ in $T$. This means, by definition, that the point $(\rho, T, o) \in \overline{m_{g}^{o}}$ is adherent to the filter $\omega$, and since $\omega$ is an ultrafilter this implies that $\omega$ converges to $(\rho, T, o)$.

Corollary 3.26 The map $\pi: \overline{m_{\Gamma}^{o}} \rightarrow \overline{m_{\Gamma}^{u}}$ is onto.

Proof Of course, $m_{\Gamma}^{o} \rightarrow m_{\Gamma}^{u}$ is onto. Now, let $T \in \partial \overline{m_{\Gamma}^{u}}$, and $\rho_{n} \in m_{\Gamma}^{o}$ be such that $\pi\left(\rho_{n}\right)$ converges to $T$. Then $\rho$ possesses a subsequence converging to some $\rho_{\infty}$, and by continuity of $\pi$ we have $\pi\left(\rho_{\infty}\right)=T$.

Remark 3.27 It is possible to write this proof without using ultrafilters (see [43]), and to prove the sequential compactness first (by considering a sequence instead of an ultrafilter on $\overline{m_{g}^{o}}$ ), and then to prove the compactness of $\overline{m_{g}^{o}}$ by using elementary general topology.

3.2.5 The space $\overline{m_{g}^{o}}$ has $4 g-3$ connected components We are now going to focus on the case when $\Gamma=\pi_{1} \Sigma_{g}$. We denote $\mathcal{T}_{g}=\mathcal{T}\left(\pi_{1} \Sigma_{g}\right)$, and $\mathcal{T}_{g}^{o}=\mathcal{T}^{o}\left(\pi_{1} \Sigma_{g}\right)$. If $(\rho, T) \in \mathcal{T}_{g}^{o}$, the set $\partial_{\infty} T$ is equipped with a total cyclic order, preserved by the action of $\pi_{1} \Sigma$, and hence it possesses an Euler class, as defined in Section 2.3. Notice that if $T$ is a line, then it follows from the definition of the Euler class that $e(\rho, T)=0$.

Theorem 3.28 The Euler class $e: m_{g}^{o} \cup \mathcal{T}_{g}^{o} \rightarrow \mathbb{Z}$ is a continuous function.

This proof will use the technical statements established in Section 2.3, which imply that we need only finitely many information about the order in order to compute the Euler class of a representation. We will be using here the notation introduced in that section.

Proof First, the set $m_{g}^{o}=\{(\rho, X) \mid \delta(X) \neq 0\}$ is open in $m_{g}^{o} \cup \mathcal{T}_{g}^{o}$, and it follows from the formula (2-1), in Section 2.3.4, that $e$ is continuous on $m_{g}^{o}$.

Now take an element $\left(\rho_{T}, T\right) \in \overline{m_{g}^{o}} \backslash m_{g}^{o}$. We shall prove that there exists a neighborhood of $T$, in the sense of the oriented equivariant Gromov topology, consisting only in representations of the same Euler class as $T$. First suppose that $T$ is not reduced to 
a line, so that it has at least three ends (in that case, $T$ possesses infinitely many ends). Take $x, y \in \partial_{\infty} T$, such that $x \notin P_{\text {ref }} \cdot y$. We may suppose that $\operatorname{Card}\left(P_{\text {ref }} \cdot y\right) \geq 2$; otherwise $\pi_{1} \Sigma_{g}$ would fix every end of $T$, and, by minimality, $T$ would be a point or a line.

Every triple $\{a, b, c\}$ of pairwise distinct elements of $P_{\text {ref }} \cdot\{x, y\}$ determines a unique class of tripods in $T$; we will denote by $P_{a b c}$ the centre of this tripod. Let $K_{1}$ be the convex hull

$$
K_{1}=\operatorname{Hull}\left\{P_{a b c} \mid(a, b, c) \in\left(P_{\text {ref }} \cdot\{x, y\}\right)^{3}, \operatorname{Card}\{a, b, c\}=3\right\} .
$$

Put $d_{T}=\max \left\{d(p, \gamma p) \mid p \in K_{1}, \gamma \in P_{\text {ref }}\right\}$ and let $d_{K_{1}}$ be the diameter of $K_{1}$. Also, for every nondegenerate triple $\{a, b, c\} \subset P_{\text {ref }} \cdot\{x, y\}$, let $P_{a b c}^{a} \in(a, b) \cap(a, c)$ be such that $d\left(P_{a b c}^{a}, P_{a b c}\right)=L$, where $L>0$ will be a sufficiently large number (see below), and let $K_{2}$ be the convex hull

$$
K_{2}=\operatorname{Hull}\left\{P_{a b c}^{a} \mid(a, b, c) \in\left(P_{\text {ref }} \cdot\{x, y\}\right)^{3}, \operatorname{Card}\{a, b, c\}=3\right\} .
$$

Finally, fix a point $p_{0} \in K_{1}$.

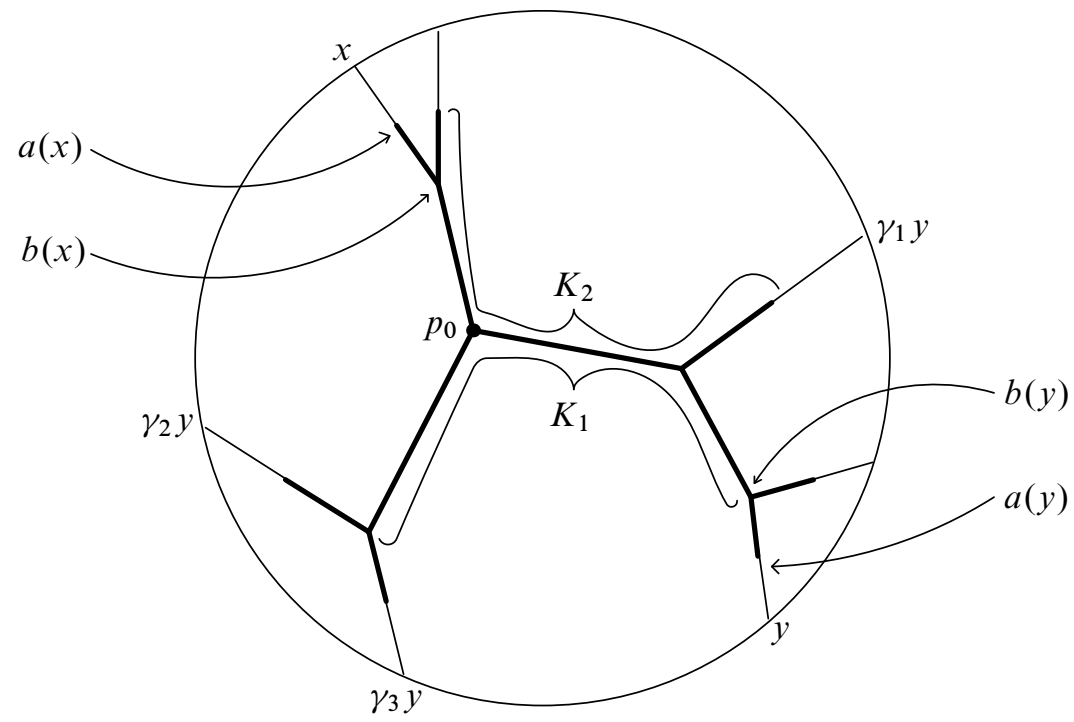

Let $a(x)$ be the closest point to $x$ in $K_{2}$. Let $b(x)$ be the projection of $a(x)$ on $K_{1}$. We define $a(y)$ and $b(y)$ similarly. We put $K=\left\{p_{0}, a(x), b(x), a(y), b(y)\right\}$ and we consider $(\rho, X) \in U_{K, \varepsilon, P_{\text {ref }}}^{\prime \prime}\left(\rho_{T}, T\right)$, where $\varepsilon>0$ will be a sufficiently small number (see below). We shall prove that $\left(\rho_{T}, T\right)$ and $(\rho, X)$ have the same Euler class, by applying Proposition 2.33. In the space $X$, denote by $p_{0}^{\prime}, a^{\prime}(y), b^{\prime}(y), a^{\prime}(x), b^{\prime}(x)$ the corresponding points. Denote by $x^{\prime} \in \partial_{\infty} X$ the end of some ray starting at $b^{\prime}(x)$ 
and passing through $\left.a^{\prime}(x)\right)$ and by $y^{\prime} \in \partial_{\infty} X$ the end of some ray starting at $b^{\prime}(y)$ and passing through $a^{\prime}(y)$ ) (chosen arbitrarily, in the case of an $\mathbb{R}$-tree).

Let $\gamma_{1}, \gamma_{2}, \gamma_{3} \in P_{\text {ref }}$ be such that $\mathbf{o}\left(\gamma_{1} x, \gamma_{2} x, \gamma_{3} y\right)=1$. We want to prove that $\mathbf{o}\left(\gamma_{1} x^{\prime}, \gamma_{2} x^{\prime}, \gamma_{3} y^{\prime}\right)=1$. For this, we shall prove the three following equalities:

$$
\begin{aligned}
\mathbf{o}\left(\gamma_{1} x, \gamma_{2} x, \gamma_{3} y\right) & =o\left(\gamma_{1} a(x), \gamma_{2} a(x), \gamma_{3} a(y)\right), \\
\mathbf{o}\left(\gamma_{1} x^{\prime}, \gamma_{2} x^{\prime}, \gamma_{3} y^{\prime}\right) & =o\left(\gamma_{1} a^{\prime}(x), \gamma_{2} a^{\prime}(x), \gamma_{3} a^{\prime}(y)\right), \\
o\left(\gamma_{1} a(x), \gamma_{2} a(x), \gamma_{3} a(y)\right) & =o\left(\gamma_{1} a^{\prime}(x), \gamma_{2} a^{\prime}(x), \gamma_{3} a^{\prime}(y)\right) .
\end{aligned}
$$

We can check that for every $\gamma \in P_{\text {ref }}, L-d_{T} \leq d\left(\gamma a(y), K_{1}\right) \leq L+d_{T}$, and similarly if we replace $y$ by $x$. Since the centre of the tripod determined by $\gamma_{1} a(x), \gamma_{2} a(x)$ and $\gamma_{3} a(y)$ lies in $K_{1}$, we have

$$
\left(\gamma_{1} a(x), \gamma_{2} a(x), \gamma_{3} a(y)\right) \in V\left(\frac{L-d_{T}}{2}\right) .
$$

If $L$ is sufficiently large and $\varepsilon$ sufficiently small, all the terms of Equations (3-6), (3-7) and (3-8) are well-defined (by Lemma 3.16), and Equation (3-8) holds (by Definition 3.19). Put $p_{1}=\gamma_{1} a(x), p_{1}^{\prime}=\gamma_{1} a^{\prime}(x), \ldots, p_{3}=\gamma_{3} a(y), p_{3}^{\prime}=\gamma_{3} a^{\prime}(y)$. Then $d_{X}\left(p_{0}^{\prime}, p_{i}^{\prime}\right)<d_{K_{1}}+L+d_{T}+\varepsilon$, and $d_{X}\left(p_{i}^{\prime}, p_{j}^{\prime}\right)>2 L-2 d_{T}-\varepsilon$, so that for every $i \in\{1,2,3\}, \sum_{j} d_{X}\left(p_{0}^{\prime}, p_{j}^{\prime}\right)<\sum_{j} d_{X}\left(p_{i}^{\prime}, p_{j}^{\prime}\right)$, provided that $L$ is large enough and $\varepsilon$ small enough. These inequalities are even finer in $T$, and by Lemma 3.16 and Remark 3.17, the equalities (3-6) and (3-7) hold. Similarly, if $\gamma_{1}, \gamma_{2}, \gamma_{3} \in P_{\text {ref }}$ are such that $\mathbf{o}\left(\gamma_{1} x, \gamma_{2} y, \gamma_{3} y\right) \neq 0$ then $\mathbf{o}\left(\gamma_{1} x^{\prime}, \gamma_{2} y^{\prime}, \gamma_{3} y^{\prime}\right)=\mathbf{o}\left(\gamma_{1} x, \gamma_{2} y, \gamma_{3} y\right)$, so that the conditions of Proposition 2.33 are satisfied. This finishes the proof, in the case when $T$ is not a line.

Now, suppose that $T$ is a line. We want to prove that there exists a neighborhood of $T$ consisting only in representations of Euler class zero. For simplicity we will prove the following:

Lemma 3.29 There exists a neighborhood $V^{\prime}$ of $T$ in which every oriented $\mathbb{R}$-tree has Euler class zero.

This lemma implies the theorem, for the following reason. For all $k \neq 0$ such that $|k| \leq 2 g-2$, denote by $L \subset \mathcal{T}_{g}^{o}$ the set of actions on lines, and denote by $F_{k} \subset L$ the set of actions on lines $(\rho, T)$ such that every neighborhood (in $\left.\overline{m_{g}^{o}}\right)$ of $(\rho, T)$ contains actions on $\mathbb{H}^{2}$ of Euler class $k$. That is, $F_{k}=L \cap \overline{m_{g, k}^{o}}$. Hence, $F_{k}$ is a closed subset of $\partial m_{g, k}^{o}$ (indeed, $L$ is a closed set, as, by definition of the topology, it is an open condition to contain a nondegenerate tripod). Now, let $(\rho, T) \in F_{k}$. By Lemma 3.29, $(\rho, T)$ has a neighborhood $V \subset \mathcal{T}_{g}^{o} \cup m_{g}^{o}$ in which every oriented $\mathbb{R}$-tree has Euler 
class 0 . Put $V^{\prime}=V \cap \partial m_{g, k}^{o}$. It is an open subset of $\partial m_{g, k}^{o}$. If there was a tree $\left(\rho^{\prime}, T^{\prime}\right) \in V^{\prime}$ not reduced to a line, then by the preceding argument, $\left(\rho^{\prime}, T^{\prime}\right)$ would have a neighborhood consisting of actions (on hyperbolic planes or on trees) of Euler class 0 , which is a contradiction since $\left(\rho^{\prime}, T^{\prime}\right) \in \overline{m_{g, k}^{o}}$. Hence, $V^{\prime}$ consists of actions on lines, ie, $F_{k}$ is open in $\partial m_{g, k}^{o}$. By Proposition 3.4, the space $m_{g, k}^{o}$ is one-ended, hence $\partial m_{g, k}^{o}$ is connected. And we can prove easily that $\partial m_{g, k}^{o}$ contains actions not reduced to a line, hence $F_{k} \neq \partial m_{g, k}^{o}$. Whence, $F_{k}=\varnothing$, for all $k \neq 0$. In other words, every action on a line has a neighborhood consisting of actions of Euler class 0; this finishes the proof of Theorem 3.28.

Proof of Lemma 3.29 Let $(\rho, T)$ be a line with $\min _{x_{0} \in T} \max _{\gamma \in S} d\left(x_{0}, \gamma \cdot x_{0}\right)=1$. Consider some point $x_{0} \in T$ realizing this minimum. Let $d_{1}$ be the greatest distance between $x_{0}$ and $\gamma x_{0}$, for every $\gamma \in P_{\text {ref }}$. Consider points $x_{1}, x_{2}, y_{1}, y_{2}$ of $T$ with $x_{1}, x_{2}$ on either side of $x_{0}$, such that $x_{i}, y_{i}$ are on the same side of $x_{0}$, such that $d\left(x_{0}, y_{i}\right)=d_{1}+4$ and $d\left(x_{0}, x_{i}\right)=2 d_{1}+6$. Let $K$ be the finite subset of $T$ consisting of $x_{1}, x_{2}, y_{1}, y_{2}$ and $P_{\text {ref }} \cdot x_{0}$. Let $\left(\rho^{\prime}, T^{\prime}\right) \in U_{K, 1 / 6, P_{\text {ref }}}^{\prime \prime}(\rho, T) \cap \mathcal{T}$, we shall prove that $e\left(\rho^{\prime}, T^{\prime}\right)=0$. If $T^{\prime}$ is a line, then there is nothing to do. Otherwise, for every point $p_{i}^{\prime} \in K^{\prime}$ approximating $K$, denote by $p_{i}^{\prime \prime}$ its projection on the segment $\left[x_{1}^{\prime}, x_{2}^{\prime}\right]$. This defines a new approximation, which realizes $\left(\rho^{\prime}, T^{\prime}\right)$ as an element of $U_{K, 1, P_{\text {ref }}^{\prime \prime}}^{\prime \prime}(\rho, T)$, and such that $K^{\prime \prime}$ is contained in a segment. Let $r$ be the end of a ray starting at $x_{0}^{\prime \prime}$ and which leaves the segment $\left[x_{1}^{\prime}, x_{2}^{\prime}\right]$ at a point $p_{0}$ at distance at most 2 of $x_{0}^{\prime \prime}$ (such a ray does exist, since $\left.\max _{\gamma \in S} d\left(x_{0}^{\prime \prime}, \gamma \cdot x_{0}^{\prime \prime}\right)<2\right)$. For every $\gamma \in P_{\text {ref }}, d\left(\gamma \cdot p_{0}, x_{0}^{\prime \prime}\right)<d_{1}+3$, and hence the segment $\left[x_{1}^{\prime}, x_{2}^{\prime}\right] \cap \operatorname{Hull}\left(P_{\text {ref }} \cdot p_{0}\right)$ is contained (strictly, on each side), in the segment $\left[y_{1}^{\prime \prime}, y_{2}^{\prime \prime}\right]$. Similarly, for every $\gamma \in P_{\text {ref }},\left[y_{1}^{\prime \prime}, y_{2}^{\prime \prime}\right] \subset\left[\gamma \cdot x_{1}^{\prime}, \gamma \cdot x_{2}^{\prime}\right]$. For $i=1,2$, let $U_{i}$ be the set of ends of rays issued from $x_{0}^{\prime \prime}$ and passing through $x_{i}^{\prime}$, and let $U_{2}^{\prime}$ be the set of ends of rays issued from $x_{0}^{\prime \prime}$ and passing through $y_{i}^{\prime \prime}$. Then $P_{\text {ref }}$ sends $U_{1} \cup U_{2}$ on a subset of $U_{1}^{\prime} \cup U_{2}^{\prime}$. Then it follows from the coherence condition on the order on $T^{\prime}$ that for all $x \in U_{1}^{\prime}, y \in U_{2}^{\prime}, \mathbf{o}(r, x, y) \in\{-1,1\}$ is constant. Suppose for instance that $\mathbf{o}(r, x, y)=1$ for all $x \in U_{1}^{\prime}, y \in U_{2}^{\prime}$. Thus, for every $\gamma \in P_{\text {ref }}$, the situation is the following.

- If $\gamma$ sends $U_{1}$ on a subset of $U_{1}^{\prime}$, and $U_{2}$ on a subset of $U_{2}^{\prime}$ (or, equivalently, if $\rho(\gamma)$ preserves the orientation of $T)$, then $\mathbf{o}(\gamma \cdot r, x, y)=1$ for all $x \in U_{1}^{\prime}$, $y \in U_{2}^{\prime}$. Denote by $A$ the set of these ends $\gamma \cdot r$.

- If $\gamma$ sends $U_{1}$ on a subset of $U_{2}^{\prime}$, and $U_{2}$ on a subset of $U_{1}^{\prime}$ (or, equivalently, if $\rho(\gamma)$ reverses the orientation of $T)$, then $\mathbf{o}(\gamma \cdot r, x, y)=-1$ for all $x \in U_{1}^{\prime}$, $y \in U_{2}^{\prime}$. Denote by $B$ the set of such ends $\gamma \cdot r$.

Now equip the set $\left\{a, u_{1}, b, u_{2}\right\}$ with the cyclic order in which we wrote them here. Denote by $h$ the order-preserving bijection which exchanges $a$ and $b$ and exchanges 
$u_{1}$ and $u_{2}$. Then we can consider the action $\pi_{1} \Sigma_{g} \rightarrow\{1, h\}$ on this ordered set, defined as follows: if $\gamma \in \pi_{1} \Sigma_{g}$ preserves the orientation of the line $T$ then we send it to 1 , otherwise we send it to $h$. Of course, this action has Euler class zero, and now it follows from Proposition 2.33, which applies here, that $e\left(\rho^{\prime}, T^{\prime}\right)=0$.

\subsection{Degeneracy of the unoriented compactification}

3.3.1 Nonorientable $\mathbb{R}$-trees Now we are going to prove that the existence of an orientation, on an $\mathbb{R}$-tree, preserved by the action of the group, is indeed a restrictive condition. More precisely:

Proposition 3.30 Let $g \geq 3$. Then the inclusion $\partial \overline{m_{g}^{u}(2)} \subset \partial \overline{m_{g}^{u}(3)}$ is strict.

Proof Of course, every isometric embedding of $\mathbb{H}^{2}$ into $\mathbb{H}^{3}$ gives rise to an embedding $m_{g}^{u}(2) \subset m_{g}^{u}(3)$ and it follows that $\partial \overline{m_{g}^{u}(2)} \subset \partial \overline{m_{g}^{u}(3)}$. In order to prove that this inclusion is strict, we shall prove that there exists an element $\left(T, \rho_{\infty}\right) \in \partial \overline{m_{g}^{u}(3)}$ such that no orientation on $T$ is preserved by $\rho_{\infty}$. Since the map $\pi: \overline{m_{g}^{o}(2)} \rightarrow \overline{m_{g}^{u}(2)}$ is onto, this implies that $\left(T, \rho_{\infty}\right) \notin \partial \overline{m_{g}^{u}(2)}$.

Consider the realization of $\pi_{1} \Sigma_{2}$ as a Fuchsian group acting on $\mathbb{H}^{2}$ with a fundamental domain as symmetric as possible, that is, a regular octagon, $\rho_{0}: \pi_{1} \Sigma_{2} \rightarrow \operatorname{PSL}(2, \mathbb{R})$.

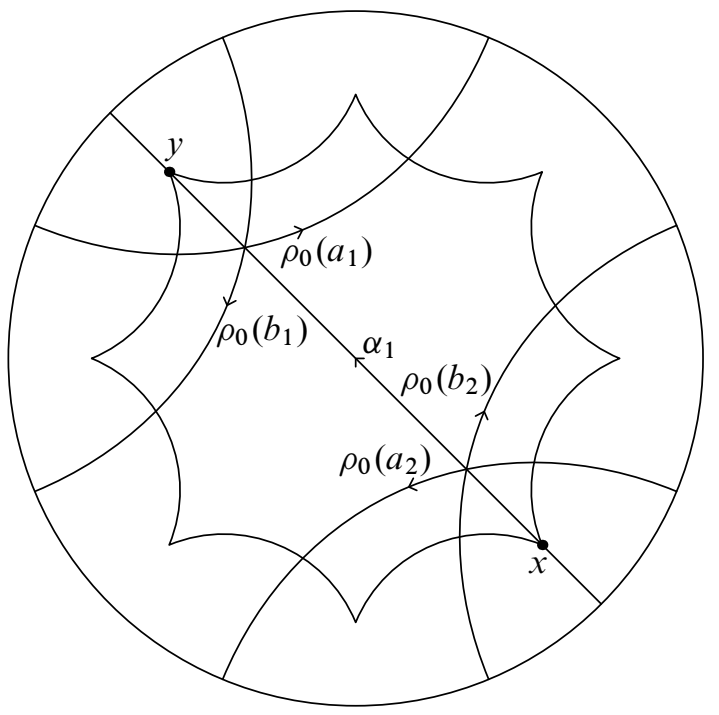

Denote by $\rho_{0}\left(a_{1}\right), \ldots, \rho_{0}\left(b_{2}\right) \in \operatorname{PSL}(2, \mathbb{R})$ the hyperbolic isometries suggested in the above picture. The element $\alpha_{1}=\rho_{0}\left(a_{1}^{-1} b_{1}^{-1} a_{2} b_{2}\right)$ is hyperbolic, of axis $(x, y)$ 
represented above (indeed, $\alpha_{1} \cdot x=y$, and if $\vec{u}$ is a unit tangent vector at $x$ pointing towards $y$, the angles of $\vec{u}$ and of its successive images with the edges of the octagon enable to check that the image of $\vec{u}$ is again a vector whose direction is the one of the axis $(x, y)$, pointing in the opposite direction of $x)$. Note that $\alpha_{1}$ is represented by a nonseparating simple closed curve on the surface $\Sigma_{2}$. Thus, we can complete the family $\left(\alpha_{1}\right)$ into $\left(\alpha_{1}, \beta_{1}, \alpha_{2}, \beta_{2}\right)$ represented by a system of curves on $\Sigma_{2}$, with $\alpha_{1}, \beta_{1}, \alpha_{2}, \beta_{2} \in \rho_{0}\left(\pi_{1} \Sigma_{2}\right)$. Define then $\rho_{n}: \pi_{1} \Sigma_{2} \rightarrow \operatorname{PSL}(2, \mathbb{R})$ by the formulas $\rho_{n}\left(a_{i}\right)=\alpha_{i}, \rho_{n}\left(b_{2}\right)=\beta_{2}, \rho_{n}\left(b_{1}\right)=\beta_{1} \alpha_{1}^{n}$, for all $n \geq 1$. Then $\rho_{n}$ is faithful and discrete, and, as $\operatorname{subgroups}$ of $\operatorname{PSL}(2, \mathbb{R})$, we have $\operatorname{Im}\left(\rho_{n}\right)=\operatorname{Im}\left(\rho_{0}\right)$. Since $\rho_{0}$ is purely hyperbolic (ie, every element of $\pi_{1} \Sigma_{2} \backslash\{1\}$ is sent to a hyperbolic element), the hyperbolic elements $\alpha_{1}, \beta_{1}$ do not share any fixed points on $\partial \mathbb{H}^{2}$. Hence $\operatorname{Tr}\left(\beta_{1} \alpha_{1}^{n}\right) \rightarrow+\infty$ as $n \rightarrow+\infty$. Now denote by $S \in \operatorname{Isom}\left(\mathbb{H}^{2}\right)$ the inversion with respect to the axis $(x, y)$ (that is, the reflection whose fixed point set is the translation axis of $\alpha_{1}$ ).

We now define $h_{n}: \pi_{1} \Sigma_{g} \rightarrow$ Isom $^{+}\left(\mathbb{H}^{3}\right)$, for every $g \geq 3$, as follows. Consider an isometric embedding $i: \mathbb{H}^{2} \hookrightarrow \mathbb{H}^{3}$; this determines an injection $\operatorname{PSL}(2, \mathbb{R}) \hookrightarrow$ Isom ${ }^{+}\left(\mathbb{H}^{3}\right)$. Every reflection in $\mathbb{H}^{2}$ can then be realized as a rotation in $\mathbb{H}^{3}$, and we denote again by $\alpha_{1}, \beta_{1}, \alpha_{2}, \beta_{2}$ and $S$ the corresponding elements of $\operatorname{Isom}^{+}\left(\mathbb{H}^{3}\right)$. We put $h_{n}\left(a_{i}\right)=\rho_{n}\left(a_{i}\right)$ and $h_{n}\left(b_{i}\right)=\rho_{n}\left(b_{i}\right)$ for $i=1,2$, and we put $h_{n}\left(a_{i}\right)=h_{n}\left(b_{i}\right)=S$ for $3 \leq i \leq g$.

We then have $h_{n} \in R_{g}(3)$, and $h_{n}\left(b_{1}\right)$ is a hyperbolic element whose translation distance tends to $+\infty$ as $n \rightarrow+\infty$, so that $\lim _{n \rightarrow+\infty} d\left(h_{n}\right)=+\infty$; hence there exists an accumulation point $\left(T, h_{\infty}\right) \in \partial \overline{m_{g}^{u}(3)}$ of this sequence of representations. We claim that no orientation on $T$ is preserved by $\rho_{\infty}$. In order to prove this, it suffices to find a nondegenerate tripod $\operatorname{Trip}(a, b, c, d) \in \operatorname{Trip}(T)$, with central point $a$, and an element $\gamma \in \pi_{1} \Sigma_{g}$, such that $\gamma(a)=a, \gamma(b)=b, \gamma(c)=d$ and $\gamma(d)=c$.

Note that when $A, B$ are hyperbolic and do not have any common fixed points in $\partial \mathbb{H}^{2}$, then the repulsive fixed point of $A B^{n}$, as $n \rightarrow+\infty$, converges to the one of $B$, whereas the attractive fixed point of $A B^{n}$ converges to the image, by $A$, of the one of $B$. Hence, the axis of $\rho_{n}\left(b_{1}\right)$ converges to some fixed geodesic line in $\mathbb{H}^{2}$. Since the images $\rho_{n}(\gamma)$ of the other generators $\gamma \in\left\{a_{1}, a_{2}, b_{2}, \ldots, a_{g}, b_{g}\right\}$ are fixed, there exists a sequence $\left(x_{0}^{n}\right)_{n} \in\left(\mathbb{H}^{2}\right)^{\mathbb{N}}$ converging to a point in $\mathbb{H}^{2}$, such that for all $n, x_{0}^{n} \in \min \left(\rho_{n}\right)$ and $i\left(x_{0}^{n}\right) \in \min \left(h_{n}\right)$. Then $d\left(i\left(x_{0}^{n}\right), h_{n}\left(a_{3}\right) \cdot i\left(x_{0}^{n}\right)\right)$ is bounded. Moreover, $h_{n}\left(b_{1}^{-1}\right)=\alpha_{1}^{-n} \beta_{1}^{-1}$. Since the axis of the symmetry $S=h_{n}\left(a_{3}\right)$ is the axis of the translation $\alpha_{1}$, we have

$$
d\left(\alpha_{1}^{-n} \beta_{1}^{-1} i\left(x_{0}^{n}\right), S . \alpha_{1}^{-n} \beta_{1}^{-1} i\left(x_{0}^{n}\right)\right)=d\left(\beta_{1}^{-1} i\left(x_{0}^{n}\right), S \beta_{1}^{-1} i\left(x_{0}^{n}\right)\right),
$$


and this number is bounded, hence the distance $d\left(h_{n}\left(b_{1}^{-1}\right) \cdot i\left(x_{0}^{n}\right), h_{n}\left(a_{3} b_{1}^{-1}\right) \cdot i\left(x_{0}^{n}\right)\right)$ is bounded. Let $x_{0}^{\infty} \in T$ be the point representing the sequence $\left(x_{0}^{n}\right)_{n \in \mathbb{N}}$. It follows that $x_{0}^{\infty}$ and $h_{\infty}\left(b_{1}^{-1}\right) \cdot x_{0}^{\infty}$ are fixed by $h_{\infty}\left(a_{3}\right)$. Thus, the segment $\left[x_{0}^{\infty}, \rho_{\infty}\left(b_{1}^{-1}\right) \cdot x_{0}^{\infty}\right]$ is globally fixed by $h_{\infty}\left(a_{3}\right)$. This segment is nondegenerate, as $\rho_{\infty}\left(b_{1}\right)$ is a hyperbolic isometry of $T$. Since $\left(h_{\infty}\left(a_{3}\right)\right)^{2}=\operatorname{Id}_{T}$, in order to prove that there is a tripod Trip $(a, b, c, d)$ such that $h_{\infty}\left(a_{3}\right)(a)=a, h_{\infty}\left(a_{3}\right)(b)=b, h_{\infty}\left(a_{3}\right)(c)=d$ and $h_{\infty}\left(a_{3}\right)(d)=c$, it suffices to prove that $h_{\infty}\left(a_{3}\right) \neq \mathrm{Id}_{T}$. This follows for instance from the fact that $h_{n}\left(a_{2} b_{1} a_{2}^{-1}\right)$ is a hyperbolic element whose fixed points in $\partial \mathbb{H}^{2}$ are distinct from those of $\alpha_{1}$ and of $h_{n}\left(b_{1}\right)$, hence for $n$ large enough the distance between $h_{n}\left(a_{2} b_{1} a_{2}^{-1}\right) \cdot i\left(x_{0}^{n}\right)$ and the axis of the symmetry $h_{n}\left(a_{1}\right)$ is of the order of $\mathrm{d}\left(h_{n}\right)$.

\subsubsection{The space $\overline{m_{g}^{u}}$ has at most 3 connected components Now we shall exhibit an-} other example of degeneracy. Fix an injective representation $\rho: \pi_{1} \Sigma_{g-1} \rightarrow \operatorname{PSL}(2, \mathbb{R})$. The circle $\mathbb{S}^{1}$ being uncountable, we can take two points $r_{0}, r_{1} \in \mathbb{S}^{1}=\partial \mathbb{H}^{2}$ such that for all $\gamma \in \pi_{1} \Sigma_{g-1}, \rho(\gamma) r_{i}=r_{j} \Leftrightarrow \gamma=1$ and $i=j$. Choose a point $x_{0}$ in the line $\left(r_{0}, r_{1}\right)$. Denote by $A_{n}$ the hyperbolic element with axis $\left(r_{0}, r_{1}\right)$ and attractive point $r_{0}$, and translation length $n$. We define a representation $\rho_{n}^{\prime}: \pi_{1} \Sigma_{g} \rightarrow \operatorname{PSL}(2, \mathbb{R})$ by letting $\rho_{n}^{\prime}\left(a_{i}\right)=\rho\left(a_{i}\right), \rho_{n}^{\prime}\left(b_{i}\right)=\rho\left(b_{i}\right)$ for $i \leq g-1$, and $\rho_{n}^{\prime}\left(a_{g}\right)=1, \rho_{n}^{\prime}\left(b_{g}\right)=A_{n}$.

Proposition 3.31 The sequence $\left(\rho_{n}^{\prime}\right)_{n} \in \overline{m_{g}^{u}} \mathbb{N}$ converges to an action $\rho_{\infty}$ on an $\mathbb{R}$-tree $T$, which does not depend on $\rho$.

In [10], J DeBlois and R Kent proved that every connected component of $R_{g-1}$ contains injective representations. It follows that this $\mathbb{R}$-tree is a common point to all the $\partial m_{g, k}^{u}$ in $\overline{m_{g}^{u}}$, for every $k \in\{0, \ldots, 2 g-4\}$, since, of course, the representation $\rho_{n}^{\prime}$ is also of Euler class $k$ (this follows immediately from Milnor's algorithm; see formula (2-1)), for all $n \in \mathbb{N}$. This proves the following result:

Corollary 3.32 Let $g \geq 2$. Then the space $\overline{m_{g}^{u}(2)}$ has at most 3 connected components. More precisely, the components of Euler class between 0 and $2 g-4$ all meet at their boundary.

Moreover, every injective representation in $R_{g-1}$ of Euler class $k$, with $|k| \leq 2 g-4$, is nonelementary (indeed, elementary subgroups of $\operatorname{PSL}(2, \mathbb{R})$ are virtually abelian, hence they do not contain isomorphic copies of $\pi_{1} \Sigma_{g-1}$ ) and nondiscrete (by W Goldman's Corollary C of [20], faithful and discrete representations have Euler class $2 g-4$ or $4-2 g$ ). It is then a consequence of Proposition 2-2 of [15] that every conjugacy class of injective representations gives rise to a distinct order. These conjugacy classes, by the theorem of DeBlois and Kent [10], have the same cardinality as $\mathbb{R}$. Hence: 
Corollary 3.33 The surjective map $\overline{m_{g}^{o}} \rightarrow \overline{m_{g}^{u}}$ has a fibre which has the cardinality of $\mathbb{R}$.

It is known (see [20]) that the space $m_{g}^{u}(3)$ has two connected components, one containing all the representations of even Euler class in $m_{g}^{u}(2)$, and the other containing those of odd Euler class. The following result follows:

Corollary 3.34 Let $g \geq 3$. Then the space $\overline{m_{g}^{u}(3)}$ is connected.

Proof of Proposition 3.31 Let $G=\pi_{1} \Sigma_{g-1} * \mathbb{Z}$. This HNN extension of $\pi_{1} \Sigma_{g-1}$ defines a Bass-Serre tree $T$, together with an action without inversions of $G$ on $T$ by isometries. The quotient of $\pi_{1} \Sigma_{g}$ by the normal subgroup generated by $a_{g}$ yields a "pinch" map $p: \pi_{1} \Sigma_{g} \rightarrow \pi_{1} \Sigma_{g-1} * \mathbb{Z}$, the generator $b_{g}$ of $\pi_{1} \Sigma_{g}$ being mapped to a generator of the $\mathbb{Z}$ factor. This defines (by composition) an action of $\pi_{1} \Sigma_{g}$ on $T$ by isometries. This action is minimal, without inversions; its kernel is exactly the normal subgroup of $\pi_{1} \Sigma_{g}$ generated by $a_{g}$. Denote it by $\rho_{\infty}$. Now we want to prove that $\left(\mathbb{H}^{2}, \rho_{n}^{\prime}\right) \rightarrow\left(T, \rho_{\infty}\right)$ in $\overline{m_{g}^{u}}$.

The action $\rho_{\infty}$ is minimal. Supposing $z$ is a generator of the $\mathbb{Z}$ factor and $c=$ $g_{1} z^{n 1} g_{2} z^{n_{2}} \cdots g_{k} z^{n_{k}}$ is an element of $\pi_{1} \Sigma_{g-1} * \mathbb{Z}$, then its translation length, with respect to $\rho_{\infty}$, is $\sum\left|n_{j}\right|$. We need only check that any accumulation point of $\left(\rho_{n}^{\prime}\right)$ is an action on a tree with these same translation lengths. If the word $c=g_{1} b_{g}^{n 1} g_{2} b_{g}^{n_{2}} \cdots g_{k} b_{g}^{n_{k}}$ is reduced, with $g_{j} \in \pi_{1} \Sigma_{g-1}$, and if $n_{k} \neq 0$, in $\mathbb{H}^{2}$, a best choice (up to constants) of starting point will be the $x_{0}$ we have chosen first, and we are going to prove that, asymptotically, the distance $(1 / n) d_{\mathbb{H}^{2}}\left(\rho_{n}^{\prime}(c) x_{0}, x_{0}\right)$ approaches $\sum_{i=1}^{m}\left|n_{k}\right|$. We work by induction on $k$. Let $c^{\prime}=g_{2} b_{g}^{n_{2}} \cdots g_{k} b_{g}^{n_{k}}$, and suppose that

$$
d\left(\rho_{n}^{\prime}\left(c^{\prime}\right) x_{0}, x_{0}\right)=n \sum_{i=2}^{k}\left|n_{i}\right|+O(1)
$$

Then $\quad d\left(\rho_{n}^{\prime}(c) x_{0}, \rho_{n}^{\prime}\left(g_{1} b_{g}^{n_{1}}\right) x_{0}\right)=n \sum_{i=2}^{k}\left|n_{i}\right|+O(1)$.

Besides

$$
d\left(\rho_{n}^{\prime}\left(g_{1} b_{g}^{n_{1}}\right) x_{0}, x_{0}\right)=d\left(A_{n}^{n_{1}} x_{0}, \rho_{n}^{\prime}\left(g_{1}^{-1}\right) x_{0}\right)=n\left|n_{1}\right|+O(1) .
$$

The isometry $\rho_{n}^{\prime}\left(g_{1} b_{g}^{n_{1}}\right)$ of $\mathbb{H}^{2}$ preserves the angles, and the angle

$$
\overline{\rho_{n}^{\prime}(c) x_{0}, \rho_{n}^{\prime}\left(g_{1} b_{g}^{n_{1}}\right) x_{0}, x_{0}}=\overline{\rho_{n}^{\prime}\left(c^{\prime}\right) x_{0}, x_{0}, \rho_{n}^{\prime}\left(b_{g}^{-n_{1}} g_{1}^{-1}\right) x_{0}}
$$

does not go to zero as $n$ goes to infinity, with $c$ fixed (indeed, $\rho_{n}^{\prime}\left(c^{\prime}\right) x_{0}$ goes to $\rho\left(g_{2}\right) r_{0}$ or $\rho\left(g_{2}\right) r_{1}$ depending on the sign of $n_{2}$, and $\rho_{n}^{\prime}\left(b_{g}^{-n_{1}} g_{1}^{-1}\right) x_{0}$ goes to $r_{0}$ 
or $r_{1}$ depending on the sign of $n_{1}$, as $n$ goes to infinity). This, combined with cosine law I, implies that

$$
d\left(\rho_{n}^{\prime}(c) x_{0}, x_{0}\right)=n \sum_{i=1}^{k}\left|n_{i}\right|+O(1),
$$

which completes the proof.

\subsubsection{The space $\overline{m_{g}^{u}}$ is connected We will now prove Corollary 1.2.}

Theorem 3.35 - For all $g \geq 2$, the space $\overline{m_{g}^{u}}$ has at most two connected components. More precisely, all the connected components, except possibly the one of Euler class $2 g-3$, meet at their boundaries.

- For all $g \geq 4$, the space $\overline{m_{g}^{u}}$ is connected.

We still consider the generating set $S=\left\{a_{1}, \ldots, b_{g}\right\}$ of $\pi_{1} \Sigma_{g}$. The main idea is the following.

Lemma 3.36 Let $\Gamma$ be a finitely generated group and let $\phi: \Gamma \rightarrow \operatorname{PSL}(2, \mathbb{R})$ be a discrete, faithful representation of cocompact image. Denote by $B_{n}$ the closed ball of radius $n$ for the Cayley metric on $\pi_{1} \Sigma_{g}$ for the generating set $S$, and suppose that $\phi_{n}: \pi_{1} \Sigma_{g} \rightarrow \Gamma$ is a noninjective morphism such that $\operatorname{ker}\left(\phi_{n}\right) \cap B_{n}=\{1\}$. Then $d\left(\phi \circ \phi_{n}\right) \rightarrow+\infty$ as $n \rightarrow+\infty$. Let $\left(T, \rho_{\infty}\right)$ be an accumulation point in $\partial \overline{m_{g}^{u}}$ of the sequence $\left(\phi \circ \phi_{n}\right)_{n \in \mathbb{N}}$. Then the action $\rho_{\infty}$ has small edge stabilizers.

Proof First, let us prove that $\lim _{n \rightarrow+\infty} d\left(\phi \circ \phi_{n}\right)=+\infty$. By contradiction, suppose that, up to extracting a subsequence, $\left(d\left(\phi \circ \phi_{n}\right)\right)_{n \in \mathbb{N}}$ converges to a real number $d \in \mathbb{R}_{+}$. Fix a point $x_{0} \in \mathbb{H}^{2}$. Since the image $\phi(\Gamma)$ is cocompact, there exist $g_{n} \in \phi(\Gamma)$ and

$$
x_{n} \in \min \left(g_{n} \cdot\left(\phi \circ \phi_{n}\right) \cdot g_{n}^{-1}\right)
$$

such that the distance $d\left(x_{0}, x_{n}\right)$ is bounded; say $d\left(x_{0}, x_{n}\right) \leq k$. Denote by $\rho_{n}$ the representation $g_{n} \cdot\left(\phi \circ \phi_{n}\right) \cdot g_{n}^{-1}$. Then for every $n \geq 0, \rho_{n}$ is discrete, and $\operatorname{ker}\left(\rho_{n}\right) \cap B_{n}=\{1\}$. For every $n \in \mathbb{N}$ and every $\gamma \in S, d\left(\rho_{n}(\gamma) \cdot x_{0}, x_{0}\right) \leq d\left(\rho_{n}\right)+2 k$, and $\lim _{n} d\left(\rho_{n}\right)=d$ hence, by Fact 2.2, up to extract it, $\left(\rho_{n}\right)_{n}$ converges to a representation $\rho \in R_{g}$. By construction, we have $\rho_{n}\left(\pi_{1} \Sigma_{g}\right) \subset \phi(\Gamma)$, hence $\rho$ is a discrete representation. And for every $\gamma \in \Gamma \backslash\{\operatorname{Id}\}$, we have $\rho_{n}(\gamma) \in \phi(\Gamma) \backslash\{\operatorname{Id}\}$ for all $n$ large enough: hence $\rho(\gamma) \neq \mathrm{Id}$, and $\rho$ is faithful. Hence, $\rho$ is discrete and faithful, hence $|e(\rho)|=2 g-2$, thus $\rho$ is the limit of representations $\rho_{n}$ which are noninjective, in particular $\left|e\left(\rho_{n}\right)\right| \neq 2 g-2$ : this is in contradiction with the continuity of the Euler class (in fact it is not necessary to use the Euler class here, but it gives the shortest proof). 
Now denote $\rho_{n}=\phi \circ \phi_{n}$. It follows that there exists an accumulation point $\left(T, \rho_{\infty}\right) \in$ $\partial \overline{m_{g}^{u}}$ of the sequence $\left(\rho_{n}\right)_{n \in \mathbb{N}}$. We still have to prove that this action has small edge stabilizers. But our representation $\rho_{n}$, for all $n \geq 0$, is discrete. We can therefore apply the same argument as M Bestvina [4] and F Paulin [35], consisting of applying Margulis' lemma. Here we follow the lines of the proof of Theorem 6.7 of [35, pages 78-79], and refer the reader to this text for more details.

Margulis' lemma There exists a constant $\mu>0$, depending only on $n$, such that for every discrete group $\Gamma$ of isometries of $\mathbb{H}^{n}$, and for all $x \in \mathbb{H}^{n}$, the subgroup generated by $\{\gamma \in \Gamma \mid d(x, \gamma x)<\mu\}$ is virtually abelian.

Let us suppose that there exists a segment $\left[x_{\infty}, y_{\infty}\right]$ of the limit $\mathbb{R}$-tree, whose stabilizer contains a free group of rank 2 . Up to considering a subgroup of index 2 , we may suppose that there exists a free group of rank $2,\langle\alpha, \beta\rangle \subset \pi_{1} \Sigma_{g}$, such that $\alpha$ and $\beta$ fix $x_{\infty}$ and $y_{\infty}$. Hence, for all $\varepsilon>0$, and for $n$ large enough, there exist $x_{n}, y_{n} \in \mathbb{H}^{2}$ such that

$$
\begin{gathered}
\left|\frac{1}{\ell\left(\rho_{n}\right)} d_{\mathbb{H}^{2}}\left(x_{n}, y_{n}\right)-1\right|<\varepsilon, \\
d_{\mathbb{H}^{2}}\left(x_{n}, \rho_{n}(\alpha) x_{n}\right)<\varepsilon \ell\left(\rho_{n}\right), \quad d_{\mathbb{H}^{2}}\left(y_{n}, \rho_{n}(\alpha) y_{n}\right)<\varepsilon \ell\left(\rho_{n}\right),
\end{gathered}
$$

and similarly for $\rho_{n}(\beta)$. Denote by $z_{n}$ the middle of the segment $\left[x_{n}, y_{n}\right]$ : it can be proved [35] that if $\varepsilon$ is small enough and if $\ell\left(\rho_{n}\right)$ is large enough, then the elements $\left[\rho_{n}(\alpha), \rho_{n}(\beta)\right]$ and $\left[\rho_{n}\left(\alpha^{2}\right), \rho_{n}(\beta)\right]$ move the point $z_{n}$ by a distance less than $\mu$. By Margulis' lemma, the elements $\rho_{n}([\alpha, \beta]), \rho_{n}\left(\left[\alpha^{2}, \beta\right]\right) \in \operatorname{PSL}(2, \mathbb{R})$ generate a virtually abelian subgroup of PSL $(2, \mathbb{R})$. But it is an easy exercise (see eg [43, Lemme 1.1.18]) to check that virtually abelian subgroups of $\operatorname{PSL}(2, \mathbb{R})$ are metabelian, ie, all the commutators commute. In particular, denote for instance

$$
w(\alpha, \beta)=\left[\left[[\alpha, \beta],\left[\alpha^{2}, \beta\right]\right],\left[[\alpha, \beta]^{2},\left[\alpha^{2}, \beta\right]\right]\right] .
$$

Then we have $\rho_{n}(w(\alpha, \beta))=1$ for all $n$ large enough. If $n$ is large enough, and larger than the length of the word $w(\alpha, \beta)$ in the generators $a_{i}, b_{i}$, this implies that $w(\alpha, \beta)=1$ in $\pi_{1} \Sigma_{g}$ (since $\left.\operatorname{ker}\left(\rho_{n}\right) \cap B_{n}=\{1\}\right)$, which contradicts the fact that $\langle\alpha, \beta\rangle$ is free. Hence, the action $\rho_{\infty}$ indeed has small edge stabilizers.

Proof of Theorem 3.35 Fix a cocompact Fuchsian group $\Gamma$, a subgroup $\mathbb{F}_{2} \subset \Gamma$ isomorphic to a free group of rank 2 , and $\phi: \Gamma \rightarrow \operatorname{PSL}(2, \mathbb{R})$ the tautological representation (the inclusion). Consider the morphism $\phi_{n}: \pi_{1} \Sigma_{g} \rightarrow \mathbb{F}_{2}$ given by Theorem 2.36, and let $\rho_{n}=\phi \circ \phi_{n}$. Then $\rho_{n}$ factors through the free group, which has a trivial $H^{2}$, hence by Remark 2.31, the representation $\rho_{n}$ has Euler class zero. By Lemma 3.36, $\left(\rho_{n}\right)_{n \in \mathbb{N}}$ possesses an accumulation point $\left(T, \rho_{\infty}\right) \in \partial \overline{m_{g}^{u}}$, which has small edge 
stabilizers. By R Skora's theorem [40], this implies that this limit is also at the boundary of the Teichmüller space; in other words, this action on an $\mathbb{R}$-tree is also the limit of representations of Euler class $2 g-2$. Hence, the closures of the connected components of $m_{g}^{u}$ of Euler classes 0 and $2 g-2$ meet. By Corollary 3.32, we already knew that the connected components of Euler classes $0,1, \ldots, 2 g-4$ meet at their boundaries; this concludes the proof of the first point.

By Proposition 4.5 of [13], the map $p_{g}: \pi_{1} \Sigma_{g} \rightarrow \operatorname{PSL}(2, \mathbb{R})$ that we defined in Section 2.4 is discrete and has Euler class $2 g-3$. We have seen (Lemma 2.37) that for all $n \geq 0$, there exists $\phi_{n} \in \operatorname{Aut}\left(\pi_{1} \Sigma_{g}\right)$ such that $\operatorname{ker}\left(p_{g} \circ \phi_{n}\right) \cap B_{n}=\{1\}$. The representation $p_{g} \circ \phi_{n}$ is still discrete, and we have $\left|e\left(p_{g} \circ \phi_{n}\right)\right|=2 g-3$, hence, as before, Lemma 3.36 ensures that the connected components of $m_{g}^{u}$ of Euler classes $2 g-3$ and $2 g-2$ meet at their boundaries.

Remark 3.37 For all $g \geq 2$, consider the action $\left(\rho_{\infty}, T\right)$ exhibited in Proposition 3.31. The representation $\rho_{\infty}: \pi_{1} \Sigma_{g} \rightarrow \operatorname{Isom}(T)$ factors through the group $\pi_{1} \Sigma_{g-1} * \mathbb{Z}$, which acts on $T$ with trivial arc stabilizers. Since there exists a morphism $\pi_{1} \Sigma_{g-1} *$ $\mathbb{Z} \rightarrow \mathbb{F}_{2}$ with nonabelian image to some free group of rank 2, as in Section 2.4 we can prove that there exist automorphisms $\phi_{n}$ of $\pi_{1} \Sigma_{g}$ such that $\operatorname{ker}\left(\rho_{\infty} \circ \phi_{n}\right) \cap B_{n}=\{1\}$. Following the proof of Lemma 5.7 of [35] (see also [35, Remark (1), page 73]), we can prove that up to extract it, the sequence $\left(\rho_{\infty} \circ \phi_{n}, T\right)$ converges to an action on an $\mathbb{R}$-tree with small stabilizers. This proves that for all $g \geq 2$ and all $k \in\{0, \ldots, 2 g-4\}$, $\overline{m_{g, k}^{u}} \cap \overline{m_{g, 2 g-2}^{u}} \neq \varnothing$.

Hence, for all $g \geq 4$ and all $k \in\{0, \ldots, 2 g-3\}$, we have $\overline{m_{g, k}^{u}} \cap \overline{m_{g, 2 g-2}^{u}} \neq \varnothing$.

3.3.4 Dynamics Finally, here we complete the proof of Theorem 1.1, which implies that the compactification $\overline{m_{g}^{u}}$ is extremely wild.

Proposition 3.38 Let $g \geq 4$ and $k \in\{0, \ldots, 2 g-3\}$. Then the boundary of the Teichmüller space embeds in $\partial m_{g, k}^{u} \subset \overline{m_{g}^{u}}$ as a closed, nowhere dense subset.

Proof Fix $g \geq 4$ and $k \in\{0, \ldots, 2 g-3\}$. Put $F_{k}=\partial m_{g, 2 g-2}^{u} \cap \partial m_{g, k}^{u}$. By Remark 3.37, we have $F_{k} \neq \varnothing$. Since $m_{g, 2 g-2}^{u}$ and $m_{g, k}^{u}$ are invariant under the (natural) action of $\operatorname{Out}\left(\pi_{1} \Sigma_{g}\right)$, it follows that $F_{k}$ is invariant, too, under this action. It is well-known (see [11, Exposé 6, Théorème VII.2, page 117]; see also [27]) that the action of $\operatorname{Out}\left(\pi_{1} \Sigma_{g}\right)$ on $\partial m_{g, 2 g-2}^{u}$ is minimal, that is, every closed subset of $\partial m_{g, 2 g-2}^{u}$, invariant under $\operatorname{Out}\left(\pi_{1} \Sigma_{g}\right)$, is either empty of is $m_{g, 2 g-2}^{u}$ itself. Since $F_{k}$ is a closed subset, this implies that $\partial m_{g, 2 g-2}^{u}=F_{k} \subset \partial m_{g, k}^{u}$.

Now denote by $G_{k}$ the boundary of $\partial m_{g, 2 g-2}^{u}$ in the space $\partial m_{g, k}^{u}$. We can easily produce elements in $\partial m_{g, k}^{u}$ which do not have small stabilizers (if $k \leq 2 g-4$ then the 
tree $\left(\rho_{\infty}, T_{\infty}\right)$ of Proposition 3.31 is an example; if $k=2 g-3$ then we can compose the map $p_{g}$ with Dehn twists along the last handle: this does not touch the kernel of the map $p_{g}$, hence this yields a sequence of actions with a fixed nontrivial kernel in $\pi_{1} \Sigma_{g}$, converging (up to extract it) to an action on an $\mathbb{R}$-tree, with this nontrivial kernel). Hence, $\partial m_{g, k}^{u} \neq \partial m_{g, 2 g-2}^{u}$. By Proposition 3.4, the space $m_{g, k}^{u}$ is connected: it follows that $G_{k} \neq \varnothing$. Since $\partial m_{g, 2 g-2}^{u}$ is closed, we have $G_{k} \subset \partial m_{g, 2 g-2}^{u}$, and $G_{k}$ is again invariant under the action of the mapping class group. So $G_{k}=\partial m_{g, 2 g-2}^{u}$.

\section{References}

[1] R C Alperin, K N Moss, Complete trees for groups with a real-valued length function, J. London Math. Soc. (2) 31 (1985) 55-68 MR810562

[2] G Baumslag, On generalised free products, Math. Z. 78 (1962) 423-438 MR0140562

[3] R Benedetti, C Petronio, Lectures on hyperbolic geometry, Universitext, Springer, Berlin (1992) MR1219310

[4] M Bestvina, Degenerations of the hyperbolic space, Duke Math. J. 56 (1988) 143-161 MR932860

[5] N Bourbaki, Éléments de mathématique. Topologie générale. Chapitres 1 à 4, Hermann, Paris (1971) MR0358652 In French

[6] D Calegari, Circular groups, planar groups, and the Euler class, from: "Proceedings of the Casson Fest", (C Gordon, Y Rieck, editors), Geom. Topol. Monogr. 7, Geom. Topol. Publ., Coventry (2004) 431-491 MR2172491

[7] C Champetier, V Guirardel, Limit groups as limits of free groups, Israel J. Math. 146 (2005) 1-75 MR2151593

[8] M Coornaert, T Delzant, A Papadopoulos, Géométrie et théorie des groupes: les groupes hyperboliques de Gromov, Lecture Notes in Math. 1441, Springer, Berlin (1990) MR1075994 In French with an English summary

[9] M Culler, P B Shalen, Varieties of group representations and splittings of 3-manifolds, Ann. of Math. (2) 117 (1983) 109-146 MR683804

[10] J Deblois, R P Kent, IV, Surface groups are frequently faithful, Duke Math. J. 131 (2006) 351-362 MR2219244

[11] A Fathi, F Laudenbach, V Poénaru, editors, Travaux de Thurston sur les surfaces, Astérisque 66-67, Soc. Math. France, Paris (1979) MR568308 Séminaire Orsay, In French with an English summary

[12] J Fresnel, Méthodes modernes en géométrie, Act. Sci. et Ind. 1437, Hermann, Paris (1998)

[13] L Funar, M Wolff, Non-injective representations of a closed surface group into PSL $(2, \mathbb{R})$, Math. Proc. Cambridge Philos. Soc. 142 (2007) 289-304 MR2314602 
[14] É Ghys, Classe d'Euler et minimal exceptionnel, Topology 26 (1987) 93-105 MR880511

[15] É Ghys, Groupes d'homéomorphismes du cercle et cohomologie bornée, from: “The Lefschetz centennial conference, Part III (Mexico City, 1984)”, (A Verjovsky, editor), Contemp. Math. 58, Amer. Math. Soc. (1987) 81-106 MR893858

[16] É Ghys, Groups acting on the circle, Enseign. Math. (2) 47 (2001) 329-407 MR1876932

[17] É Ghys, P de la Harpe (editors), Sur les groupes hyperboliques d'après Mikhael Gromov, Progress in Math. 83, Birkhäuser, Boston (1990) MR1086648 Papers from the Swiss Seminar on Hyperbolic Groups held in Bern, 1988

[18] W M Goldman, Discontinuous groups and the Euler class, PhD thesis, University of California Berkeley (1980)

[19] W M Goldman, The symplectic nature of fundamental groups of surfaces, Adv. in Math. 54 (1984) 200-225 MR762512

[20] W M Goldman, Topological components of spaces of representations, Invent. Math. 93 (1988) 557-607 MR952283

[21] V Guirardel, Approximations of stable actions on $\mathbf{R}$-trees, Comment. Math. Helv. 73 (1998) 89-121 MR1610591

[22] V Guirardel, Limit groups and groups acting freely on $\mathbb{R}^{n}$-trees, Geom. Topol. 8 (2004) 1427-1470 MR2119301

[23] N Hitchin, The self-duality equations on a Riemann surface, Proc. London Math. Soc. (3) 55 (1987) 59-126 MR887284

[24] M Kapovich, B Leeb, On asymptotic cones and quasi-isometry classes of fundamental groups of 3-manifolds, Geom. Funct. Anal. 5 (1995) 582-603 MR1339818

[25] S Katok, Fuchsian groups, Chicago Lectures in Math., Univ. of Chicago Press (1992) MR1177168

[26] B Leeb, A characterization of irreducible symmetric spaces and Euclidean buildings of higher rank by their asymptotic geometry, Bonner Math. Schriften 326, Univ. Bonn Math. Inst. (2000) MR1934160 arXiv:0903.0584

[27] H Masur, Ergodic actions of the mapping class group, Proc. Amer. Math. Soc. 94 (1985) 455-459 MR787893

[28] S Matsumoto, Some remarks on foliated $S^{1}$ bundles, Invent. Math. 90 (1987) 343-358 MR910205

[29] C T McMullen, Ribbon $\mathbb{R}$-trees and holomorphic dynamics on the unit disk, J. Topol. 2 (2009) 23-76 MR2499437

[30] J Milnor, On the existence of a connection with curvature zero, Comment. Math. Helv. 32 (1958) 215-223 MR0095518 
[31] J W Morgan, Group actions on trees and the compactification of the space of classes of $\mathrm{SO}(n, 1)$-representations, Topology 25 (1986) 1-33 MR836721

[32] J W Morgan, P B Shalen, Valuations, trees, and degenerations of hyperbolic structures. I, Ann. of Math. (2) 120 (1984) 401-476 MR769158

[33] A Parreau, Compactification d'espaces de représentations de groupes de type fini, preprint (2010) Available at http://www-fourier.ujf-grenoble.fr/ parreau

[34] A Parreau, Espaces de représentations complètement réductibles, J. London Math. Soc. 83 (2011) 545-562

[35] F Paulin, Topologie de Gromov équivariante, structures hyperboliques et arbres réels, Invent. Math. 94 (1988) 53-80 MR958589

[36] F Paulin, The Gromov topology on R-trees, Topology Appl. 32 (1989) 197-221 MR1007101

[37] F Paulin, Sur la compactification de Thurston de l'espace de Teichmüller, from: "Géométries à courbure négative ou nulle, groupes discrets et rigidités", (L Bessières, A Parreau, B Rémy, editors), Sémin. Congr. 18, Soc. Math. France, Paris (2009) 421-443 MR2655319

[38] V Poénaru, Groupes discrets, Lecture Notes in Math. 421, Springer, Berlin (1974) MR0407155

[39] Z Sela, Diophantine geometry over groups. I: Makanin-Razborov diagrams, Publ. Math. Inst. Hautes Études Sci. 93 (2001) 31-105 MR1863735

[40] R K Skora, Splittings of surfaces, J. Amer. Math. Soc. 9 (1996) 605-616 MR1339846

[41] W P Thurston, On the geometry and dynamics of diffeomorphisms of surfaces, Bull. Amer. Math. Soc. (N.S.) 19 (1988) 417-431 MR956596

[42] A Weil, Remarks on the cohomology of groups, Ann. of Math. (2) 80 (1964) 149-157 MR0169956

[43] M Wolff, Sur les composantes exotiques des espaces d'actions de groupes de surfaces sur le plan hyperbolique, $\mathrm{PhD}$ thesis, Université de Grenoble I (2007) Available at http://www.math.jussieu.fr/ wolff

[44] J W Wood, Bundles with totally disconnected structure group, Comment. Math. Helv. 46 (1971) 257-273 MR0293655

Institut de Mathématiques de Jussieu, Université Pierre et Marie Curie - Paris 6

Case 247, 4 place Jussieu, Fr-75005 Paris, France

wolff@math.jussieu.fr

http://www.math.jussieu.fr/ wolff

Proposed: Walter Neumann

Received: 8 August 2008

Seconded: Ronald J Stern, Danny Calegari

Revised: 20 April 2011 